A class of noncommutative projective surfaces

\author{
Rogalski, D. and Stafford, J T
}

2009

MIMS EPrint: 2007.213

Manchester Institute for Mathematical Sciences

School of Mathematics

The University of Manchester

\footnotetext{
Reports available from: http://eprints.maths.manchester.ac.uk/

And by contacting: The MIMS Secretary

School of Mathematics

The University of Manchester

Manchester, M13 9PL, UK
} 


\title{
A class of noncommutative projective surfaces
}

\author{
D. Rogalski and J. T. Stafford
}

\begin{abstract}
Let $A=\bigoplus_{i \geqslant 0} A_{i}$ be a connected graded, noetherian $k$-algebra that is generated in degree one over an algebraically closed field $k$. Suppose that the graded quotient ring $Q(A)$ has the form $Q(A)=k(X)\left[t, t^{-1} ; \sigma\right]$, where $\sigma$ is an automorphism of the integral projective surface $X$. Then we prove that $A$ can be written as a naïve blowup algebra of a projective surface $\mathbb{X}$ birational to $X$. This enables one to obtain a deep understanding of the structure of these algebras; for example, generically they are not strongly noetherian and their point modules are not parametrized by a projective scheme. This is despite the fact that the simple objects in qgr- $A$ will always be in (1-1) correspondence with the closed points of the scheme $\mathbb{X}$.
\end{abstract}

\section{Contents}

1. Introduction

1.1. The results

1.2. An example

1.3. History

1.4. The proofs

1.5. Questions

2. Point modules

3. Birationally commutative domains

4. Contracted points

5. The stable scheme and its birational map

6. Birationally geometric algebras

7. Constructing the automorphism

8. Naïve blowing up

9. Surjectivity in large degree

10. The main theorem

11. Examples

References

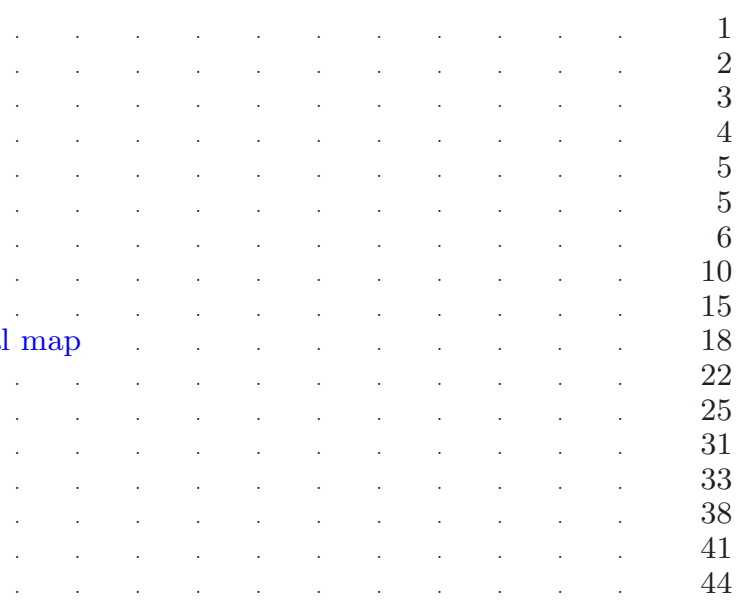

\section{Introduction}

One of the outstanding problems of noncommutative projective geometry is to classify all noncommutative projective surfaces. There are many ways of phrasing this problem; from a ring-theoretic standpoint it means classifying (hopefully in some geometric way) those connected graded rings that should be thought of as corresponding to surfaces. In this paper, we will achieve just such a classification for a large class of noncommutative rings: noetherian connected graded rings that are birationally 2-geometric, as defined below.

Received 23 November 2007; revised 7 October 2008.

2000 Mathematics Subject Classification 14A22, 16P40, 16P90, 16S38, 16W50, 18E15 (primary).

The first author was partially supported by NSF grants DMS-0202479 and DMS-0600834 while the second author was partially supported by NSF grants DMS-0245320 and DMS-0555750 and also by the Leverhulme Research Interchange Grant F/00158/X. Part of this work was written up while the second author was visiting and supported by the Newton Institute, Cambridge. We would like to thank all three institutions for their financial support. 


\subsection{The results}

Let us first give the statement of the main theorem and then explain its significance and applications. Throughout, $k$ will be an algebraically closed base field. A $k$-algebra $A$ is called connected graded (cg) if $A=\bigoplus_{n \geqslant 0} A_{n}$, where $A_{0}=k$ and $\operatorname{dim}_{k} A_{n}<\infty$ for each $n$. For a noetherian cg domain $A$, one can invert the nonzero homogeneous elements to obtain the graded quotient ring $Q(A) \cong D\left[t, t^{-1} ; \sigma\right]$, for some automorphism $\sigma$ of the division ring $D=Q(A)_{0}$ (see also Section 3). We say that $A$ is birationally geometric if $D \cong k(V)$, the field of rational functions on an integral projective scheme $V$, and $\sigma$ is induced from an automorphism of $V$. If in addition $V$ is a surface, we say that $A$ is birationally 2-geometric.

The aim of the paper is to classify birationally 2 -geometric algebras as naïve blowup algebras, in the sense of [16]. These are described as follows. Let $X$ be an integral projective scheme, with zero-dimensional subscheme $Z=Z_{\mathcal{I}}=\operatorname{Spec} \mathcal{O}_{X} / \mathcal{I}$, automorphism $\sigma$ and $\sigma$-ample invertible sheaf $\mathcal{L}$, as defined in Section 8. In a manner reminiscent of the Rees ring construction of the blowup of a commutative scheme, we form the bimodule algebra

$$
\mathcal{R}=\mathcal{R}(X, Z, \mathcal{L}, \sigma)=\mathcal{O}_{X} \oplus \mathcal{J}_{1} \oplus \mathcal{J}_{2} \oplus \ldots,
$$

where $\mathcal{J}_{n}=\mathcal{L}_{n} \otimes_{\mathcal{O}_{X}} \mathcal{I}_{n}$ for $\mathcal{I}_{n}=\mathcal{I} \cdot \sigma^{*} \mathcal{I} \cdot \ldots \cdot\left(\sigma^{*}\right)^{n-1} \mathcal{I}$. As is described in detail in [16] and explained briefly here in Section 8, this bimodule algebra has a natural multiplication and the naïve blowup algebra of $X$ at $Z$ is then defined to be the algebra of sections

$$
R=R(X, Z, \mathcal{L}, \sigma)=\mathrm{H}^{0}(X, \mathcal{R})=k \oplus \mathrm{H}^{0}\left(X, \mathcal{J}_{1}\right) \oplus \mathrm{H}^{0}\left(X, \mathcal{J}_{2}\right) \oplus \ldots
$$

Although implicitly $Z$ is nonempty, this definition makes perfect sense when $Z=\emptyset$, in which case $B(X, \mathcal{L}, \sigma)=R(X, \emptyset, \mathcal{L}, \sigma)$ is just the twisted homogeneous coordinate ring that is so important in other aspects of noncommutative projective geometry; see, for example, [6] or [25]. For any zero-dimensional subscheme $Z \subset X$ there is a natural embedding $R=R(X, Z, \mathcal{L}, \sigma) \hookrightarrow$ $B=B(X, \mathcal{L}, \sigma)$ and the multiplication in $R$ is also that induced from $B$.

We can now state the main result of this paper (see Theorem 10.1 for a more detailed version).

Main Theorem 1.1. Let $A$ be a $c g$ noetherian domain that is generated in degree one and birationally 2-geometric. Then, up to a finite-dimensional vector space, either $A \cong B(\mathbb{X}, \mathcal{L}, \sigma)$ or $A \cong R(\mathbb{X}, Z, \mathcal{L}, \sigma)$, for some projective surface $\mathbb{X}$ with automorphism $\sigma \in \operatorname{Aut}(\mathbb{X}), \sigma$-ample invertible sheaf $\mathcal{L}$ and zero-dimensional subscheme $Z$.

Noetherian naïve blowup algebras only appear when the algebras are far from commutative; explicitly for each closed point $z \in Z$ the orbit $\langle\sigma\rangle \cdot z$ will be critically dense in the sense that the orbit is infinite and all of its infinite subsets are Zariski dense in $\mathbb{X}$. One consequence of this observation is the following result.

Corollary 1.2 (Corollary 10.9). Let $A$ be a $\mathrm{cg}$ noetherian domain that is generated in degree one and birationally 2-geometric. If $A$ satisfies a polynomial identity then there exists a twisted homogeneous coordinate ring $B=B(\mathbb{X}, \mathcal{L}, \sigma)$ such that $A \subseteq B$ with $\operatorname{dim}_{k} B / A<\infty$.

Combined with the results from $[\mathbf{1 6}, \mathbf{2 3}]$, the alternative $A \cong R(\mathbb{X}, Z, \mathcal{L}, \sigma)$ in Theorem 1.1 has remarkable consequences for the structure of the algebra $A$ and its representations. Before giving these results we need some further definitions. The category of noetherian $\mathbb{Z}$-graded right $A$-modules, with homomorphisms being graded homomorphisms of degree zero, will be denoted by gr- $A$. Let tors- $A$ denote the full subcategory of $\operatorname{gr}-A$ consisting of modules of finite length, 
with quotient category qgr- $A=\operatorname{gr}-A /$ tors- $A$. (Thinking of qgr- $A$ as the category of coherent sheaves on the imaginary scheme Proj $A$ can often provide useful intuition.) $A$ point module for $A$ is a cyclic graded $A$-module $M=\bigoplus_{n \geqslant 0} M_{n}$ such that $\operatorname{dim}_{k} M_{n}=1$ for all $n$. A point module in qgr- $A$ is defined to be the image in qgr- $A$ of a cyclic graded $A$-module $M=\bigoplus_{n \geqslant 0} M_{n}$, generated in degree zero, such that $\operatorname{dim}_{k} M_{n}=1$ for all $n \gg 0$. For point modules defined over arbitrary base rings and the formalities on parametrization, see Section 2.

Corollary 1.3. Let $A$ be as in Theorem 1.1 and keep the notation from that result.

(I) If $A \cong B(\mathbb{X}, \mathcal{L}, \sigma)$ then:

(a) $A$ is strongly noetherian; that is, $A \otimes_{k} C$ is noetherian for all commutative noetherian $k$-algebras $C$;

(b) $\operatorname{qgr}-A \simeq \operatorname{coh} \mathbb{X}$, the category of coherent sheaves on $\mathbb{X}$;

(a) the set of point modules for $A$ (both in $\operatorname{gr}-A$ and in qgr- $A$ ) is parametrized by the scheme $\mathbb{X}$;

(c) $A$ has a balanced dualizing complex in the sense of $[\mathbf{2 9}]$.

(II) Otherwise $A \cong R(\mathbb{X}, Z, \mathcal{L}, \sigma)$, for some nonempty finite subscheme $Z$ and:
(a) $A$ is not strongly noetherian;
(b) neither the point modules in gr- $A$ nor those in qgr- $A$ are parametrized by a scheme of locally finite type;
(c) the simple objects in qgr- $A$ are the point modules and are naturally in (1-1) correspondence with the closed points of $\mathbb{X}$;
(d) qgr- $A$ has finite cohomological dimension;
(e) $A$ does not have a balanced dualizing complex;
(f) $H^{1}(A)=\operatorname{Ext}_{\mathrm{qgr}-A}^{1}(A, A)$ is infinite-dimensional;
(g) A does not satisfy generic flatness in the sense of [2].

Proof. This is really just a case of quoting the literature. To be precise, part (Ia) follows from [2, Proposition 4.13], while part (Ib) is [6, Theorem 1.3] and (Id) is proved in [29, Theorem 7.3]. Part (Ic) follows from [24, Theorem 1.1, 1.2] and [16, Proposition 10.2]. All the results stated in part (II) are contained in [23, Theorem 1.1 and Remark 1.2].

We should emphasize the striking fact that the properties described by part (II) of the corollary are not exceptional: as soon as $(\mathbb{X}, \sigma)$ has at least one critically dense orbit then the theorem and its corollary imply that, generically, each noetherian cg subalgebra of $k(\mathbb{X})\left[t, t^{-1} ; \sigma\right]$ has these properties. We would also point out that, despite these curious features, the category qgr- $A$ from Corollary 1.3(II) still has many pleasant features; notably it is surprisingly similar to the category of coherent sheaves on a projective scheme. This is illustrated by the following result, which also shows that the properties of the naïve blowup qgr- $A$ are actually closer to those of the original scheme $\mathbb{X}$ than they are to those of the classical blowup $\widetilde{\mathbb{X}}$ of $\mathbb{X}$ at $Z$.

Proposition 1.4 ([23, Theorem 4.10]). Keep the hypotheses of Theorem 1.1. Then the subcategory of (Goldie) torsion objects in $\operatorname{qgr}-R(\mathbb{X}, Z, \mathcal{L}, \sigma)$ is equivalent to the category of torsion coherent $\mathcal{O}_{\mathbb{X} \text {-modules. }}$

\subsection{An example}

Here is an easy example that illustrates the types of algebras that appear in Theorem 1.1 and its corollary (see, also, Example 4.3). 
A typical example of a twisted coordinate ring is the 'quantum polynomial ring' $S=S_{p q}$ with generators $x, y, z$ and relations $x y-p y x, x z-q z x$ and $y z-q p^{-1} z y$ for $p$ and $q \in k^{*}$. This can also be written as $S \cong B\left(\mathbb{P}^{2}, \mathcal{O}_{\mathbb{P}}(1), \sigma\right)$, where $\sigma \in \operatorname{Aut}\left(\mathbb{P}^{2}\right)$ is defined by the formula $\left(\lambda_{0}: \lambda_{1}: \lambda_{2}\right) \mapsto\left(\lambda_{0}: p \lambda_{1}: q \lambda_{2}\right)$. It follows that $Q(S)_{0}=k\left(y x^{-1}, z x^{-1}\right)=k\left(\mathbb{P}^{2}\right)$ and so, in this special case, Theorem 1.1 classifies the noetherian cg algebras $A$ with $Q(A)=Q(S)$. To see an explicit example of case (II) of the corollary, assume that the scalars $p, q$ are algebraically independent over the base field, take $Z$ to be the single reduced point $(1: 1: 1)$ and consider the ring $R\left(\mathbb{P}^{2}, Z, \mathcal{O}(1), \sigma\right)$; it is easy to show this ring is equal to $k\langle x-y, x-z\rangle \subseteq S$.

It is also easy to find cg subalgebras of $Q\left(S_{p q}\right)$ that are not noetherian. For example, [16, Proposition 4.8] implies that, for this choice of $\sigma$, the naïve blowup algebra $R\left(\mathbb{P}^{2}, Z^{\prime}, \mathcal{O}(1), \sigma\right)$ is not noetherian when $Z^{\prime}=\{(1: 0: 0)\}$. For a detailed and more algebraic examination of these rings; see $[\mathbf{2 0}]$.

\subsection{History}

We briefly explain the history behind these results and their wider relevance.

As we remarked earlier, it is useful to think of qgr- $A$ for a noetherian cg ring $A$ as coherent sheaves on the nonexistent scheme Proj $A$. Extending this analogy further one can think of a noncommutative projective integral curve and surface as ggr- $A$ for a noetherian $\operatorname{cg}$ domain $A$ with Gelfand-Kirillov dimension two, and three, respectively. For the purposes of this discussion we will also restrict ourselves to algebras generated in degree one (see Section 1.5 for comments about the general case). Under this analogy, noncommutative integral projective curves have been classified in $[\mathbf{3}]$ (they are nothing more than $\operatorname{qgr}-B(X, \mathcal{L}, \sigma) \simeq \operatorname{coh} X$ for integral projective curves $X)$. Similarly, noncommutative projective planes and their algebraic analogues, the Artin-Schelter regular rings with the Hilbert series of a polynomial ring in three variables, have been classified in $[\mathbf{4}, \mathbf{5}, \mathbf{9}]$. Such an Artin-Schelter regular ring either equals $B\left(\mathbb{P}^{2}, \mathcal{O}(1), \sigma\right)$ for some $\sigma$ or has a factor isomorphic to $B(E, \mathcal{L}, \sigma)$ for a projective curve $E$ in which case $(E, \sigma)$ determines $A$. These results are surveyed in $[\mathbf{2 5}]$, and the reader is referred to that paper for more details.

Motivated by these results, in [1] Artin posed the problem of classifying all noncommutative integral projective surfaces and this paper was motivated in part by that question. In one sense, however, Theorem 1.1 is orthogonal to his question, since he was interested in finding the noncommutative algebras whose properties are close to those of commutative integral surfaces. For example, he included the existence of a balanced dualizing complex as one of his hypotheses! Of course, if $A$ satisfies the hypotheses of Theorem 1.1 and also has a balanced dualizing complex, then Corollary 1.3(IIe) implies that $A$ is a twisted homogeneous coordinate ring, in which case the category qgr- $A$ of coherent sheaves on the corresponding noncommutative projective scheme is commutative: $\mathrm{qgr}-A \simeq \operatorname{coh} \mathbb{X}$.

Further motivation for this paper comes from recent work on strongly noetherian algebras. These algebras are studied in detail in $[\mathbf{2}]$ and $[\mathbf{8}]$ and have many pleasant properties, some of which are described in Section 1.4 below. One is therefore interested in understanding when cg algebras satisfy this property and for birationally geometric algebras this is answered by the following result.

Theorem 1.5 ([24, Theorem 1.2] and [2, Proposition 4.13]). (i) Suppose that $A$ is a strongly noetherian, $\mathrm{cg}$ domain that is generated in degree one and birationally geometric. Then, up to a finite-dimensional vector space, $A \cong B(\mathbb{X}, \mathcal{L}, \sigma)$, for a projective variety $\mathbb{X}$, with automorphism $\sigma \in \operatorname{Aut}(\mathbb{X})$ and $\sigma$-ample invertible sheaf $\mathcal{L}$.

See (ii) Conversely, if $\mathcal{L}$ is a $\sigma$-ample invertible sheaf on a projective scheme $X$, then $B(X, \mathcal{L}, \sigma)$ is strongly noetherian. 
Since noetherian cg domains that are not strongly noetherian exist in profusion, even among domains of Gelfand-Kirillov dimension three, it is important to understand their structure and Theorem 1.1 gives one step in that direction.

\subsection{The proofs}

We next want to give the strategy behind the proof of the main theorem, but we first outline the proof of Theorem 1.5(i) as the contrast is illuminating. Strongly noetherian rings are known to have a number of very pleasant properties and in particular one has the following result from [8, Theorem E4.3]: If $A$ is a strongly noetherian $k$-algebra then the point modules for $A$ are parametrized by a projective scheme $\mathbb{X}$. The key step in the proof of Theorem 1.5 is that, under the hypotheses of the theorem and up to a finite-dimensional vector space as always, there exists a surjection $\chi: A \rightarrow B(\mathbb{X}, \mathcal{L}, \sigma)$ for this scheme $\mathbb{X}$ together with a sheaf $\mathcal{L}$ and automorphism $\sigma$. By hypothesis $Q(A)_{0}=k(V)$ for some scheme $V$ and the next step is to regard $Q(A)_{\geqslant 0}=\sum_{n \geqslant 0} k(V) t^{n}$ as a 'generic point module' with coefficients from $k(V)$ (see Section 3 for a discussion of this type of module). The existence of this generic point module can then be used to show that $\chi$ is injective.

Now suppose that $A$ is merely noetherian. As we have seen, the point modules for $A$ are no longer parametrized by a projective scheme and so there is no easy candidate for the scheme $\mathbb{X}$; indeed the construction of $\mathbb{X}$ forms a major part of the proof of the theorem. The idea is as follows. A truncated point module of length $n+1$ is a cyclic graded $A$-module $M=\bigoplus_{i=0}^{n} M_{i}$ where $\operatorname{dim}_{k} M_{i}=1$ for each $i$. By [4, Proposition 3.9] these modules are parametrized by a scheme $W_{n}$ and there are natural maps $\Phi_{n-1}, \Psi_{n-1}: W_{n} \rightarrow W_{n-1}$ given by further truncation: $\Phi_{n-1}(M)=M / M_{n}$, respectively, $\Psi_{n-1}(M)=M_{\geqslant 1}[1]$, where [1] denotes the shift in gradation. When $A$ is strongly noetherian, it follows from [8, Corollary E4.5] that $\Phi_{n}$ and $\Psi_{n}$ become isomorphisms for $n \gg 0$. The scheme $\mathbb{X}$ of Theorem 1.5 is then just $\mathbb{X}=W_{n}$ for such an $n$, with automorphism $\sigma=\Psi_{n} \Phi_{n}^{-1}$. In contrast, when $A$ is only noetherian these maps will never be isomorphisms. Fortunately there is a reduced and irreducible component $Y_{n}$ of $W_{n}$ such that (a) $\Phi_{n}$ and $\Psi_{n}$ restrict to maps between the $Y_{n}$ and (b) $k\left(Y_{n}\right) \cong k(V)$ and so there is an analogous generic truncated point module $\bigoplus_{i=0}^{n} Q(A)_{i}$ (see Corollary 3.5).

The main step the proof of Theorem 1.1 is to show that, for $n \gg 0$, one can appropriately blow down $\pi: Y_{n} \rightarrow \mathbb{X}$ to give a scheme $\mathbb{X}$ for which the induced action of the birational map $\sigma=\Psi_{n} \Phi_{n}^{-1}$ is actually an automorphism. This is the scheme appearing in the theorem. The scheme $\mathbb{X}$ appears in a second way, which also indicates how it is constructed: given a truncated point module $M^{\prime}$ corresponding to some $y \in Y_{n}$ and $n \gg 0$, write $M^{\prime}$ as the image of a (non-unique) point module $M$ and take the image $\bar{M}$ of $M$ in qgr- $A$. As Corollary 7.11 shows, it is this object that is uniquely determined by $M^{\prime}$ and the map $\pi$ corresponds to mapping $M^{\prime} \mapsto \bar{M}$. The proof of these assertions takes up Sections $2-7$. Once $\mathbb{X}$ has been constructed, one can use the ideas from $[\mathbf{3}, \mathbf{1 6}, \mathbf{2 4}]$ to prove the theorem (see Sections 8-10).

\subsection{Questions}

We end the introduction with some comments and questions. First, Theorem 1.5 is actually stronger than stated since it works for any $\mathrm{cg}$ noetherian domain $A$ that is birationally commutative in the sense that $Q(A)_{0}$ is a finitely generated field; in other words one does not need to assume that the automorphism $\sigma$ is induced from that of a scheme. We conjecture that, for surfaces, Theorem 1.1 also holds under this more general hypothesis, but there is one case we are as yet unable to handle. Using work of Diller and Favre [10] which, essentially, classifies birational maps of surfaces one has the following result. 
Theorem $1.6([\mathbf{2 2}])$. Let $Q=K\left[t, t^{-1} ; \sigma\right]$, where $K$ is a finitely generated field extension of $k$ of transcendence degree two. Then every $\mathrm{cg}$ Ore domain $A$ with graded quotient ring $Q$ has the same Gelfand-Kirillov dimension $d \in\{3,4,5, \infty\}$. If $d<\infty$, then $d=3$ or 5 if and only if $\sigma$ is induced from an automorphism of some projective surface $X$ with $K \cong k(X)$. If $d=\infty$ then $A$ is not noetherian.

This just leaves the case when $d=4$. This definitely can occur; for example, take the automorphism $u \mapsto u v, v \mapsto v$ of $k\left(\mathbb{P}^{2}\right)=k(u, v)$. We believe, however, that when $d=4$ the algebra $A$ can never be noetherian, which would solve the conjecture. See Example 11.3 for a typical example in this dimension. Note, also, that the case $d=5$ is covered by Theorem 1.1 and so noncommutative surfaces are a little more general than algebras of Gelfand-Kirillov dimension 3.

We end with two further questions. First, as is true of Theorem 1.5, is there a version of Theorem 1.1 describing any birationally geometric $A$ ? Second, in the present paper we always assume that our algebras are generated in degree one. In the commutative case this is not so much of a restriction since one can always reduce to that case by taking an appropriate Veronese ring. In the noncommutative case, however, it is a more severe restriction, since it excludes the idealizer examples that appear in $[\mathbf{3}, \mathbf{2 1}, \mathbf{2 6}]$. It would be interesting to know whether, as happened for curves in [3], one can classify all noetherian cg algebras that are birationally 2-geometric in terms of variants of naïve blowup algebras and idealizers.

\section{Point modules}

The method we use to introduce geometry into the study of noncommutative algebras is the theory of (truncated) point modules and the schemes that parametrize them. In this section we recall the relevant definitions and prove some basic facts about these objects that will be needed in the sequel. These concepts were first introduced by Artin, Tate and Van den Bergh in $[\mathbf{4}$, Section 3], and more details can be found both there and in [24, Section 4]. For the most part our geometric notation is standard and, unless we say otherwise, we will use the definitions given in [14].

As in the introduction, fix a cg algebra $A=\bigoplus_{n \geqslant 0} A_{n}$ with categories gr- $A$ and qgr- $A$. The category of all graded right $A$-modules is written $\operatorname{Gr}-A$, but we will not need the corresponding quotient category Qgr- $A$. Assume for the rest of the section that $A$ is generated by $A_{1}$ as a $k$-algebra. Given a commutative $k$-algebra $R$ we write $A_{R}=A \otimes_{k} R$, regarded as a graded $R$-algebra by putting $R$ into degree zero. Following [4, Definition 3.8], a truncated $R$-point module of length $n+1$, for $n \geqslant 0$, is a graded cyclic right $A_{R}$-module $M=\bigoplus_{i=0}^{n} M_{i}$ where $M_{0}=R$ and each $M_{i}$ is a locally free $R$-module of rank 1 . An $R$-point module is given by the same definition, except that now $n=\infty$. Under the identification of $R$ with $R^{\text {op }}$, we can and usually will regard (truncated) right $R$-point modules as $(R, A)$-bimodules since this will frequently make their module structures more transparent. Note that if $M$ is a (truncated) $R$-point module, then the identification $M_{0}=R$ defines a unique isomorphism $M \cong A_{R} / I$ for some right ideal $I$, and so this rigidifies the given (truncated) $R$-point module. A (truncated) $k$-point module is just called a (truncated) point module. Write $\mathcal{P}(R)$ for the set of isomorphism classes of right $R$-point modules and $\mathcal{P}_{n}(R)$ for the set of isomorphism classes of truncated right $R$-point modules of length $n+1$. We write $\mathcal{P}=\mathcal{P}(k), \mathcal{P}_{n}=\mathcal{P}_{n}(k)$ and let $\mathcal{P}^{\ell}(R), \ldots, \mathcal{P}_{n}^{\ell}$ denote the left-hand analogues of each of these sets.

Recall that Yoneda's Lemma embeds the category Schemes of noetherian $k$-schemes inside the category of functors from the category Alg of commutative noetherian $k$-algebras to the category Sets of sets, where a scheme $X$ corresponds to the functor $h_{X}: R \mapsto \operatorname{Hom}(\operatorname{Spec} R, X)$ 
$($ see $[\mathbf{1 2}$, Section VI]). A functor $h: \mathbf{A l g} \rightarrow$ Sets is said to be represented by the scheme $X$ if $h$ and $h_{X}$ are isomorphic functors.

We will apply this theory to the functors arising from truncated point modules over our algebra $A$. By [4, Proposition 3.9] the functor $F_{n}: \mathbf{A l g} \rightarrow$ Sets defined by $R \mapsto \mathcal{P}_{n}(R)$ is represented by a (commutative) projective scheme $W_{n}$ called the $n^{\text {th }}$ truncated point scheme for $A$. Since it will be used many times, we recall the functorial property of $F_{n}$. Thus, let $M \in$ $\mathcal{P}_{n}(R)$ be a truncated $R$-point module corresponding to a morphism of schemes $\theta: \operatorname{Spec} R \rightarrow$ $W_{n}$ and let $\alpha: R \rightarrow S$ be a homomorphism of commutative noetherian $k$-algebras. The induced map Spec $S \rightarrow \operatorname{Spec} R$ will be denoted by $\alpha^{\star}$. Using our convention that $R$-point modules are written as $(R, A)$-bimodules, $S \otimes_{R} M$ is naturally a truncated point module over $A_{R} \otimes_{R} S \cong$ $A_{S}$ and the morphism of schemes Spec $S \rightarrow W_{n}$ corresponding to $S \otimes_{R} M$ is simply $\theta \alpha^{\star}$. If we need to remember the map $\alpha$ in this construction, we will write $S_{\alpha} \otimes M$ for $S \otimes_{R} M$.

Following [4] the schemes $W_{n}$ can be constructed explicitly. Write $A \cong T\left(A_{1}\right) / J$, where $J=\bigoplus_{n \geqslant 0} J_{n}$ is a homogeneous ideal of the tensor algebra $T\left(A_{1}\right)$. Let $\mathbb{P}$ denote the projective space $\mathbb{P}\left(A_{1}^{*}\right)$ of lines in $A_{1}^{*}$ and for each $n \geqslant 1$ write $\mathbb{P}^{\times n}$ for the product of $n$ copies of $\mathbb{P}$. For $n \geqslant 0$, we identify $T_{n}$ with the global sections of the sheaf $\mathcal{O}(1,1, \ldots, 1)$ on $\mathbb{P}^{\times n}$. Under this identification, $J_{n}$ defines a vector subspace $\widetilde{J}_{n}$ of $\mathrm{H}^{0}\left(\mathbb{P}^{\times n}, \mathcal{O}(1,1, \ldots, 1)\right)$ and, by the proof of $\left[\mathbf{4}\right.$, Proposition 3.9], $W_{n}$ can be identified with the vanishing locus of $\widetilde{J}_{n}$ in $\mathbb{P}^{\times n}$. We typically write this embedding as $\iota_{n}: W_{n} \hookrightarrow \mathbb{P}^{\times n}$. For each $n$ and $1 \leqslant a \leqslant b \leqslant n$ let $\pi_{a, b}: \mathbb{P}^{\times n} \rightarrow \mathbb{P}^{\times(b-a+1)}$ denote the projection onto the coordinates between $a$ and $b$ inclusively. By [4, Proposition 3.5], $\pi_{1, n}\left(W_{n+1}\right) \subseteq W_{n}$ and $\pi_{2, n+1}\left(W_{n+1}\right) \subseteq W_{n}$ and we define projective morphisms $\Phi_{n}: W_{n+1} \rightarrow W_{n}$ and $\Psi_{n}: W_{n+1} \rightarrow W_{n}$ by restricting $\pi_{1, n}$, respectively, $\pi_{2, n+1}$ to $W_{n+1}$. We call the collection of schemes and morphisms $\left(W_{n}, \Phi_{n}, \Psi_{n}\right)$ the point scheme data for the algebra $A$, where the embeddings $\iota_{n}$ are to be understood.

The morphisms $\Phi_{n}$ and $\Psi_{n}$ are closely related to truncations of modules. Let $R$ be a commutative noetherian $k$-algebra and, for an $R$-module $V$, set $V^{*}=\operatorname{Hom}_{R}(V, R)$. If $M=$ $\bigoplus M_{i} \in \operatorname{Gr}-A$ and $n \in \mathbb{Z}$, then the shift $M[n]$ of $M$ is defined by $M[n]_{r}=M_{n+r}$. Given a truncated $R$-point module of length $n+2$, say $M=\bigoplus_{i=0}^{n+1} M_{i} \in \mathcal{P}_{n+1}(R)$, we define the right truncation of $M$ to be

$$
\Phi_{n}^{\prime}(M)=M / M_{n+1}=M_{0} \oplus \ldots \oplus M_{n} \in \mathcal{P}_{n}(R),
$$

and we define the left truncation of $M$ to be the right $A_{R}$-module

$$
\Psi_{n}^{\prime}(M)=M_{1}^{*} \otimes_{R} M[1]_{\geqslant 0}=\left(M_{1}^{*} \otimes_{R} M_{1}\right) \oplus \ldots \oplus\left(M_{1}^{*} \otimes_{R} M_{n+1}\right) \in \mathcal{P}_{n}(R) .
$$

The canonical identification $M_{1}^{*} \otimes_{R} M_{1}=R$ ensures that $\Psi_{n}^{\prime}(M)$ really is a truncated $R$-point module.

By repeating the previous few paragraphs with left modules in place of right modules, we can define the analogous left point scheme data $\left(W_{n}^{\ell}, \Phi_{n}^{\ell}, \Psi_{n}^{\ell}\right)$. For example, $W_{n}^{\ell}$ is a projective scheme which represents the functor $F_{n}^{\ell}: \mathbf{A l g} \rightarrow$ Sets defined by $R \mapsto \mathcal{P}_{n}^{\ell}(R)$. As is shown by the next lemma, $\left(W_{n}^{\ell}, \Phi_{n}^{\ell}, \Psi_{n}^{\ell}\right)$ is a mirror of $\left(W_{n}, \Phi_{n}, \Psi_{n}\right)$ and so we will not need to consider it separately.

For the rest of this section, we analyse the morphisms $\Phi_{n}$ and $\Psi_{n}$ and begin with some elementary properties that will be used frequently.

Lemma 2.3. (i) For $n \geqslant 0$, the maps $\mathcal{P}_{n+1}(R) \rightarrow \mathcal{P}_{n}(R)$ given by the rules $M \mapsto \Phi_{n}^{\prime}(M)$, and $M \mapsto \Psi_{n}^{\prime}(M)$ induce the respective morphisms $\Phi_{n}: W_{n+1} \rightarrow W_{n}$ and $\Psi_{n}: W_{n+1} \rightarrow W_{n}$ between the representing schemes.

(ii) There are isomorphisms $\gamma_{n}: W_{n} \stackrel{\sim}{\longrightarrow} W_{n}^{\ell}$ for $n \geqslant 0$ satisfying $\gamma_{n}^{-1} \Phi_{n}^{\ell} \gamma_{n+1}=\Psi_{n}$ and $\gamma_{n}^{-1} \Psi_{n}^{\ell} \gamma_{n+1}=\Phi_{n}$.

(iii) For all $n \geqslant 0$ we have $\Psi_{n} \Phi_{n+1}=\Phi_{n} \Psi_{n+1}$. 
Proof. (i) See the proof of [4, Proposition 3.9].

(ii) Fix $n \geqslant 0$ and suppose that $M=\bigoplus_{i=0}^{n} M_{i} \in \mathcal{P}_{n}(R)$, for some noetherian commutative $k$-algebra $R$. Define $M^{\vee}=\left(\bigoplus_{i=-n}^{0} M_{-i}^{*} \otimes_{R} M_{n}\right)[-n]$ as a variant of the Matlis dual and note that $M^{\vee}$ is a graded left $A_{R}$-module, under the convention that $A$ acts from the left and $R$ from the right. By [16, Proposition 10.2(1)] $M^{\vee} \in \mathcal{P}_{n}^{\ell}(R)$ (the reader should note that the proof in [16] omitted the term $\otimes M_{n}$ which is need to ensure that $M_{1}^{\vee}=R$ ).

Similarly, if $N \in \mathcal{P}_{n}^{\ell}(R)$, then $N^{\vee}=\left(\bigoplus_{i=-n}^{0} N_{n} \otimes_{R} N_{-i}^{*}\right)[-n] \in \mathcal{P}_{n}(R)$. The two operations are inverses, compatible with change of rings, and so the map $M \mapsto M^{\vee}$ induces an isomorphism $\gamma_{n}: W_{n} \stackrel{\sim}{\longrightarrow} W_{n}^{\ell}$ between the representing schemes.

Given $M=\bigoplus_{i=0}^{n+1} M_{i} \in \mathcal{P}_{n+1}(R)$, then a short calculation shows that

$$
\left(\left(M^{\vee}\right)_{\leqslant n}\right)^{\vee} \cong \bigoplus_{i=0}^{n} M_{1}^{*} \otimes_{R} M_{i+1}=\Psi_{n}^{\prime}(M) \in \mathcal{P}_{n}(R),
$$

and this isomorphism is compatible with change of rings. Using part (i) and its left-sided analogue we conclude that $\gamma_{n}^{-1} \Phi_{n}^{\ell} \gamma_{n+1}=\Psi_{n}$ as maps from $W_{n+1}$ to $W_{n}$. The proof that $\gamma_{n}^{-1} \Psi_{n}^{\ell} \gamma_{n+1}=\Phi_{n}$ is analogous.

(iii) The morphisms $\pi_{1, n} \pi_{2, n+2}$ and $\pi_{2, n+1} \pi_{1, n+1}$ give the same projection $\mathbb{P}^{\times(n+2)} \rightarrow \mathbb{P}^{\times n}$. Restriction to the subscheme $W_{n} \subseteq \mathbb{P}^{n}$ gives $\Phi_{n} \Psi_{n+1}=\Psi_{n} \Phi_{n+1}$.

In order to avoid any possible ambiguity we will clarify our conventions about a morphism of schemes $f: Z \rightarrow W$. If we say that $f^{-1}$ is defined at a point $w \in W$, then we do mean that it is defined as a morphism from an open neighbourhood of $w$ to an open neighbourhood of the (necessarily singleton) set $f^{-1}(w)$. As such, $f^{-1}$ automatically defines a local isomorphism at $w$, meaning that it defines an isomorphism from an open neighbourhood of $w$ to an open neighbourhood of $f^{-1}(w)$. Finally, we adopt the usual convention that an automorphism $\tau$ acts on functions by $(\tau(\theta))(z)=\theta(\tau(z))$ for $z \in Z$.

Lemma 2.4. Let $f: Z \rightarrow W$ be a proper morphism of finite type schemes and suppose that $w \in W$ is a closed point with set-theoretic fibre $f^{-1}(w)=\{z\}$ a single point. Then:

(i) if the induced map $f_{z}^{\sharp}: \mathcal{O}_{W, w} \rightarrow \mathcal{O}_{Z, z}$ is an isomorphism, then the map $f^{-1}$ is a local isomorphism at $w$;

(ii) if $f_{*}\left(\mathcal{O}_{Z}\right)=\mathcal{O}_{W}$, then the map $f^{-1}$ is a local isomorphism at $w$.

Proof. (i) It is routine to show that, since $f_{z}^{\sharp}$ is an isomorphism, there are open neighbourhoods $w \in V \subseteq W$ and $z \in U \subseteq Z$ such that $f$ restricts to an isomorphism $U \rightarrow V$. Since the fibre $f^{-1}(w)=\{z\}$ is a singleton and $f$ is a closed morphism, we can shrink $V$ to a smaller open set $V^{\prime} \ni w$ whose inverse image satisfies $f^{-1}\left(V^{\prime}\right) \subseteq U$. Then $f$ is injective over $V^{\prime}$ and $f^{-1}$ is defined at $w$.

(ii) Since $f_{*}\left(\mathcal{O}_{Z}\right)=\mathcal{O}_{W}$, the map $f_{z}^{\sharp}$ can be identified with the map

$$
\widehat{f}: \underset{w \in V}{\lim _{w}} \mathcal{O}_{Z}\left(f^{-1}(V)\right) \longrightarrow \underset{z \in U}{\lim } \mathcal{O}_{Z}(U),
$$

where $U$ and $V$ run through open sets in their respective schemes. As in part (i), for any such $U$ containing $z$ there is an open set $V$ containing $w$ with $f^{-1}(V) \subseteq U$. It follows that $\widehat{f}$ is an isomorphism and so the result follows from part (i).

It follows from [8, Theorem E4.3] together with Lemma 2.3 that the point scheme data $\left(W_{n}, \Phi_{n}, \Psi_{n}\right)$ stabilizes for strongly noetherian rings, in the sense that $\Phi_{n}$ and $\Psi_{n}$ are isomorphisms for large $n$. In contrast, as we remarked in the introduction, this need not be the case for arbitrary noetherian rings. Fortunately, as we show in Proposition 2.5, the point 
scheme data does at least stabilize locally, and this will form the starting point of many of the results in this paper.

In the next few results, we let $\chi$ be a symbol which denotes either $\Phi$ or $\Psi$; thus any property claimed for a map $\chi_{n}$ is supposed to hold for both $\Phi_{n}$ and $\Psi_{n}$.

Proposition 2.5. Assume that $A$ is a noetherian, $c g$-algebra generated in degree one and pick $n_{0} \geqslant 0$. Let $\left\{w_{n} \in W_{n}: n \geqslant n_{0}\right\}$ be a sequence of (not necessarily closed) points such that $\chi_{n}\left(w_{n+1}\right)=w_{n}$ for all $n \geqslant n_{0}$. Then, for all $n \gg n_{0}$, the fibre $\chi_{n}^{-1}\left(w_{n}\right)=\left\{w_{n+1}\right\}$ is a singleton and $\chi_{n}^{-1}$ is a local isomorphism at $w_{n}$.

Proof. Assume that $\chi=\Phi$; the proof for $\chi=\Psi$ follows similarly using left modules and Lemma 2.3(ii).

Suppose first that the points $\left\{w_{n} \in W_{n}: n \geqslant n_{0}\right\}$ are closed points. Then this sequence $\left\{w_{n}\right\}$ corresponds to a right $k$-point module $M=A / J$; thus $J$ is a right ideal with $\operatorname{dim}_{k} J_{n}=$ $\operatorname{dim}_{k} A_{n}-1$ for all $n \geqslant 0$. Choose $m_{0} \geqslant n_{0}$ such that $J$ is generated in degrees at most $m_{0}$. If possible, pick $m \geqslant m_{0}$ such that the fibre $\Phi_{m}^{-1}\left(w_{m}\right)$ contains (at least) two distinct closed points, say $w_{m+1}$ and $y$, corresponding to the truncated point modules $M_{\leqslant(m+1)}$ and $N$. Write $N=A / I$ for some right ideal $I$ and notice that $I_{\leqslant m}=J_{\leqslant m}$ but $I_{m+1} \neq J_{m+1}$. However, as $A$ is generated in degree one and $J$ is generated in degrees at most $m$, this implies that $I_{m+1} \supseteq I_{m} A_{1}=J_{m} A_{1}=J_{m+1}$. Since $\operatorname{dim}_{k} A_{m+1} / I_{m+1}=1=\operatorname{dim} A_{m+1} / J_{m+1}$, this forces $I_{m+1}=J_{m+1}$, giving a contradiction.

Thus $\Phi_{n}^{-1}\left(w_{n}\right)=\left\{w_{n+1}\right\}$ for all $n \gg n_{0}$. By [4, Proposition 3.6(ii)] and the last line of the proof of that result, the induced map of noetherian local rings $\widehat{\Phi}_{n}: \mathcal{O}_{W_{n}, w_{n}} \rightarrow \mathcal{O}_{W_{n+1}, w_{n+1}}$ is surjective for any such $n$. For sufficiently large $n$, the map $\widehat{\Phi}_{n}$ is therefore an isomorphism. By [14, Corollary II.4.8(e)] $\Phi_{n}$ is proper and so Lemma 2.4(i) implies that $\Phi_{n}^{-1}$ is a local isomorphism at $w_{n}$.

Now suppose that the points $\left\{w_{n} \in W_{n}: n \geqslant n_{0}\right\}$ are not necessarily closed and write $\bar{w}_{n}$ for the closure of $w_{n}$. By properness, $\Phi_{n}\left(\bar{w}_{n+1}\right)$ is closed, and thus equal to $\bar{w}_{n}$, for any $n \geqslant n_{0}$. Thus, beginning with any closed point $y_{n_{0}} \in \bar{w}_{n_{0}}$, we can choose a sequence of closed points $y_{n} \in \bar{w}_{n}$ such that $\Phi_{n}\left(y_{n+1}\right)=y_{n}$, for each $n \geqslant n_{0}$. For $n \gg n_{0}$ the first part of the proof shows that the fibre $\Phi_{n}^{-1}\left(y_{n}\right)=\left\{y_{n+1}\right\}$ is a singleton and that $\Phi_{n}^{-1}$ is a local isomorphism at $y_{n}$. But then $\Phi_{n}^{-1}$ is then also local isomorphism at $w_{n}$ and so certainly $\Phi_{n}^{-1}\left(w_{n}\right)=w_{n+1}$ is a singleton for all such $n$.

For $m>n \geqslant 0$ define morphisms $\Phi_{m, n}$ and $\Psi_{m, n}$ by $\Phi_{m, n}=\Phi_{n} \Phi_{n+1} \cdot \ldots \cdot \Phi_{m-1}: W_{m} \longrightarrow W_{n} \quad$ and $\quad \Psi_{m, n}=\Psi_{n} \Psi_{n+1} \cdot \ldots \cdot \Psi_{m-1}: W_{m} \longrightarrow W_{n}$.

Corollary 2.7. Fix $n_{0} \geqslant 0$. For each $n \geqslant n_{0}$ let $G_{n}$ be a finite set of (not necessarily closed) points of $W_{n}$ such that $\chi_{n}\left(G_{n+1}\right) \subseteq G_{n}$. Then for $n \gg n_{0}$, the cardinality $\left|G_{n}\right|$ is constant and $\chi_{n}$ gives a bijection between $G_{n+1}$ and $G_{n}$. Moreover, if $g \in G_{n}$ for any such $n$, then $\chi_{n}^{-1}$ is a local isomorphism at $g$.

Proof. As before, it suffices to prove the result for $\chi=\Phi$. For each $n \geqslant n_{0}$, let $H_{n}$ be the subset of points $w \in G_{n}$ for which there exists $m>n$ and two distinct points $y, z \in G_{m}$ such that $\Phi_{m, n}(y)=w=\Phi_{m, n}(z)$. Obviously $\Phi_{n}\left(H_{n+1}\right) \subseteq H_{n}$, so if $H_{n+1} \neq \emptyset$ for some $n>n_{0}$, then $H_{n} \neq \emptyset$. Assume that $H_{n} \neq \emptyset$ for all $n \geqslant n_{0}$. Then we claim that there exists a sequence of points $\left\{w_{n} \in H_{n}: n \geqslant n_{0}\right\}$ such that $\Phi_{n}\left(w_{n+1}\right)=w_{n}$ for all $n \geqslant n_{0}$. To see this, for $w \in H_{n}$, 
set $\ell(w)=\sup \left\{r: w=\Phi_{r+n, n}(y)\right.$ for some $\left.y \in H_{n+r}\right\}$. As $H_{n_{0}}$ is finite we may pick $w_{n_{0}} \in$ $H_{n_{0}}$ with $\ell\left(w_{n_{0}}\right)$ maximal. If $\ell\left(w_{n_{0}}\right)=t<\infty$, then $H_{j}=\emptyset$ for all $j>t+n_{0}$, contradicting our assumption. So $\ell\left(w_{n_{0}}\right)=\infty$. Now we may construct the sequence $w_{n}$ inductively: having chosen $w_{n} \in H_{n}$ with $\ell\left(w_{n}\right)=\infty$, since $H_{n+1}$ is finite there exists $w_{n+1} \in H_{n+1}$ such that $\Phi_{n}\left(w_{n+1}\right)=w_{n}$ and $\ell\left(w_{n+1}\right)=\infty$.

Thus if $H_{n} \neq \emptyset$ for all $n \geqslant n_{0}$, there is a sequence of points $w_{n} \in W_{n}$ such that (i) $\Phi_{n}\left(w_{n+1}\right)=$ $w_{n}$ for all $n \geqslant n_{0}$, and (ii) for infinitely many choices of $n$ the set theoretic fibre $\Phi_{n}^{-1}\left(w_{n}\right)$ is not a singleton. This contradicts Proposition 2.5. So $H_{n}$ is empty for $n \gg n_{0}$ and $\Phi_{n}$ induces an injection from $G_{n+1}$ to $G_{n}$ for all such $n$. Since each $G_{n}$ is finite, this implies that $\Phi_{n}$ must induce a bijection for $G_{n+1}$ to $G_{n}$ for all $n \gg n_{0}$. The final statement of the corollary now follows from the final assertion of Proposition 2.5.

\section{Birationally commutative domains}

Fix a noetherian cg $k$-algebra $A$ that is generated in degree one, with point scheme data $\left(W_{n}, \Phi_{n}, \Psi_{n}\right)$. In this section we examine how the ideal structure of $A$ is reflected in the varietal structure of the schemes $W_{n}$. For the algebras $A$ of interest, this will allow us to isolate subschemes $Y_{n} \subseteq W_{n}$ that better reflect the geometry of $A$.

If $A$ is also a domain then [19, C.I.1.6 and A.I.4.3] shows that the set $\mathcal{C}$ of nonzero homogeneous elements of $A$ forms an Ore set with corresponding localization $Q(A)=A_{\mathcal{C}} \cong$ $D\left[t, t^{-1} ; \tau\right]$. Here $\tau$ is an automorphism of the division ring $D$, and $D\left[t, t^{-1} ; \tau\right]$ is the twisted Laurent polynomial ring defined by $t d=d^{\tau} t$ for all $d \in D$. We call $Q(A)$ the graded quotient ring of $A$ and $D$ the function division ring of $A$.

If $D$ is a field, we call $A$ birationally commutative, and note that in this case $D$ will be a finitely generated field extension of $k$; see, for example, Corollary 3.5(i). (One reason for the notation is that, if $A$ were the homogeneous coordinate ring of an integral projective scheme $Y$, then $D$ would just be the function field $k(Y)$.)

We begin with a useful observation about the point scheme data for factor rings.

Lemma 3.1. Let $A$ be a noetherian cg $k$-algebra, generated in degree one, with a homogeneous ideal I. Let $\left(W_{n}, \Phi_{n}, \Psi_{n}\right)$ be the point scheme data for $A$, and let $\left(\widehat{W}_{n}, \widehat{\Phi}_{n}, \widehat{\Psi}_{n}\right)$ be the point scheme data for $A / I$. Then, for all $n \geqslant 0, \widehat{W}_{n}$ is canonically identified with a closed subscheme of $W_{n}$ with $\widehat{\Phi}_{n}=\left.\Phi_{n}\right|_{\widehat{W}_{n}}$ and $\widehat{\Psi}_{n}=\left.\Psi_{n}\right|_{\widehat{W}_{n}}$, respectively.

Moreover, as sets, $\Phi_{n}^{-1}\left(\widehat{W}_{n}\right)=\widehat{W}_{n+1}$ and $\Psi_{n}^{-1}\left(\widehat{W}_{n}\right)=\widehat{W}_{n+1}^{n}$ for all $n \gg 0$.

Proof. We are given a presentation $A=T\left(A_{1}\right) / J$ so that $W_{n} \subseteq \mathbb{P}^{\times n}=\mathbb{P}\left(A_{1}^{*}\right)^{\times n}$ is the zero set $\mathcal{V}\left(\widetilde{J}_{n}\right)$ of the multi-linearization $\widetilde{J}_{n}$ of $J_{n}$. Now, $A / I \cong T / K$ for some ideal $K \supseteq J$ and so $\widehat{W}_{n}$ is defined to be a subscheme of $\mathbb{P}\left(\left(A_{1} / K_{1}\right)^{*}\right)^{\times n}$. However, we can identify $\mathbb{P}\left(\left(A_{1} / K_{1}\right)^{*}\right)^{\times n}$ with a closed subscheme of $\mathbb{P}\left(A_{1}^{*}\right)^{\times n}$ via the embedding $\left(A_{1} / K_{1}\right)^{*} \hookrightarrow A_{1}^{*}$, after which $\widehat{W}_{n}$ is the zero set of the larger vector space $\widetilde{K}_{n} \supseteq \widetilde{J}_{n}$. Thus $\widehat{W}_{n}$ is indeed closed in $W_{n}$. (If one considers the corresponding point modules, the embedding $\widehat{W}_{n} \hookrightarrow W_{n}$ is nothing more than the identification of point modules over $A / K$ with the point modules over $A$ annihilated by $K$.) Since $\Phi_{n}$ and $\Psi_{n}$ are defined as restrictions of projection maps $\mathbb{P}^{\times(n+1)} \rightarrow \mathbb{P}^{\times n}$, the maps $\widehat{\Phi}_{n}$ and $\widehat{\Psi}_{n}$ are just further restrictions of those maps.

Assume that $I$ is generated, as a right $A$-module, in degrees at most $n_{0}$; thus $I_{n} A_{1}=I_{n+1}$ for $n \geqslant n_{0}$. For $n \geqslant n_{0}$, let $z \in \widehat{W}_{n}$ and $y \in W_{n+1}$ be closed points with $\Phi_{n}(y)=z$. Thus $z$ corresponds to a truncated point module $A / L$ where $L$ is a right ideal of $A$ containing $I$ and $y$ corresponds to a truncated point module $A / M$ with $M_{\leqslant n}=L_{\leqslant n} \supseteq I_{\leqslant n}$. As $n \geqslant n_{0}$ this 
implies that $M \supseteq M_{\leqslant n} A \supseteq I$ and hence that $y \in \widehat{W}_{n+1}$. In other words, $\Phi_{n}^{-1}\left(\widehat{W}_{n}\right)=\widehat{W}_{n+1}$ for all $n \geqslant n_{0}$. The assertion $\Psi_{n}^{-1}\left(\widehat{W}_{n}\right)=\widehat{W}_{n+1}$ follows by working with left modules and using Lemma 2.3(ii).

If $A$ is a strongly noetherian domain which is birationally commutative, then it is isomorphic in large degree to a twisted homogeneous coordinate $\operatorname{ring} B=B(X, \mathcal{L}, \sigma)$ (see Section 1.5 or [24, Theorem 1.2]) and there is a natural correspondence between ideals of $A$ and $\sigma$-invariant subschemes $Z \subseteq X$ (see [3, Lemma 4.4]). If $A$ is merely noetherian then the correspondence, which forms the content of the next proposition, is more subtle, basically because the morphisms $\Psi_{n}$ and $\Phi_{n}$ need not be isomorphisms for $n \gg 0$.

We begin by discussing the point modules defined by function division rings of factors of $A$. Let $B=\bigoplus B_{n}$ be a birationally commutative cg algebra that is generated in degree one, with graded quotient $\operatorname{ring} Q(B)=K\left[t, t^{-1} ; \sigma\right]$ for some field $K$ and $\sigma \in \operatorname{Aut}(K)$. Since $B_{1} \neq 0$ we may assume that $t \in B_{1}$, as is often convenient in these situations. Regard $\mathcal{G}=K[t ; \sigma]=$ $Q(B)_{\geqslant 0}$ as a right $B_{K}$-module, where $B$ acts by right multiplication but $K$ acts from the left. Since $t \in B_{1}, \mathcal{G}$ is cyclic and hence is a $K$-point module for $B$. Now consider the shift $\mathcal{G}[1]_{\geqslant 0}$ of $\mathcal{G}$. It is immediate that the map $\mu: \mathcal{G} \rightarrow \mathcal{G}[1]_{\geqslant 0}$, given by $t^{m} \ell \mapsto t^{m+1} \ell$ for $\ell \in K$, is an isomorphism of graded right $B$-modules. However, this is not an isomorphism of $B_{K}$-modules since the $K$-action is twisted: given $a \in K$ and $g=t^{m} \ell \in \mathcal{G}_{\geqslant 0}$ for some $\ell \in K$, then the left $K$-action is given by

$$
a \circ \mu(g)=a t^{m+1} \ell=t^{m+1} a^{\sigma^{-(m+1)}} \ell=\mu\left(a^{\sigma^{-1}} \circ\left(t^{m} \ell\right)\right)=\mu\left(a^{\sigma^{-1}} \circ g\right) .
$$

There is a second way of writing the module $\mathcal{G}[1]_{\geqslant 0}$. In the notation from Section 2 , consider the homomorphism $\sigma: K=R \rightarrow K=S$ and the resulting $B_{S}$-module $K_{\sigma} \otimes \mathcal{G}=S \otimes_{R} \mathcal{G}$. (We have introduced $R$ and $S$ in order to distinguish between the two copies of $K$.) As right $B$ modules, $\mathcal{G} \cong S \otimes_{R} \mathcal{G}$ under the map $\lambda: g \mapsto 1 \otimes g$. However, the $S$-module action is again twisted: if $s * r=s r^{\sigma}$ denotes the action of $r \in R$ on $s \in S$ then

$$
a \circ \lambda(g)=a \otimes g=\left(1 * a^{\sigma^{-1}}\right) \otimes g=1 \otimes a^{\sigma^{-1}} g=\lambda\left(a^{\sigma^{-1}} \circ g\right) \text { for } a \in K=S \text { and } g \in \mathcal{G} .
$$

Comparing the two displayed equations shows the following.

Lemma 3.2. Keep the notation from the previous paragraph. Then $\mathcal{G}[1]_{\geqslant 0} \cong K_{\sigma} \otimes \mathcal{G}$ as $A_{K}$-modules.

Proposition 3.3. Let $A$ be a noetherian $c g k$-algebra that is generated in degree one. Then there is a bijection between the following sets.

(i) Completely prime, homogeneous ideals $P$ of $A$ (excluding $A_{\geqslant 1}$ ) such that $A / P$ is birationally commutative.

(ii) Sequences of (not necessarily closed) points $\left\{\eta_{n} \in W_{n}: n \gg 0\right\}$ such that $\Phi_{n}\left(\eta_{n+1}\right)=$ $\Psi_{n}\left(\eta_{n+1}\right)=\eta_{n}$ for all $n \gg 0$, where two such sequences are identified if they agree for all large $n$.

Under this correspondence the function division ring $Q(A / P)_{0}$ in part (i) is isomorphic to the field $k\left(\eta_{n}\right)$ for $n \gg 0$ in part (ii).

Proof. Step 1: Suppose that $P$ is a completely prime ideal as in (i). Let $\bar{A}=A / P$ and write $Q(\bar{A}) \cong K\left[t, t^{-1} ; \sigma\right]$, where $K$ is a field and $t \in \bar{A}_{1}$. The idea is to think of $\mathcal{G}=Q(\bar{A})_{\geqslant 0}$ as a 'generic' point module for $\bar{A}$. More precisely, regard $\mathcal{G}$ as a $K$-point module for $A$, as discussed above. For $n \geqslant 1$, the module $\mathcal{G}_{\leqslant n}=\bigoplus_{i=0}^{n} K t^{i}$ is a truncated $K$-point module of length $n+1$ 
for $A$ and so corresponds to a morphism $\theta_{n}$ : Spec $K \rightarrow W_{n}$. By Lemma 2.3(i), $\Phi_{n} \theta_{n+1}=\theta_{n}$ for all $n \gg 0$.

Lemma 2.3(i) also implies that the left truncation $\Psi_{n}^{\prime}\left(\mathcal{G}_{\leqslant n+1}\right)=\bigoplus_{i=0}^{n} \mathcal{G}[1]_{i}$ corresponds to the morphism $\Psi_{n} \theta_{n+1}:$ Spec $K \rightarrow W_{n}$ for $n \gg 0$. By Lemma 3.2, $\Psi_{n}^{\prime}\left(\mathcal{G}_{\leqslant n+1}\right) \cong K_{\sigma} \otimes \mathcal{G}_{\leqslant n}$ and so $\Psi_{n} \theta_{n+1}=\theta_{n} \sigma^{\star}$, where $\sigma^{\star}: \operatorname{Spec} K \rightarrow \operatorname{Spec} K$ is the morphism induced by $\sigma$. If $\eta_{n}=\theta_{n}(\eta) \in W_{n}$ denotes the image of the point $\eta=\operatorname{Spec} K$ then $\Psi_{n}\left(\eta_{n+1}\right)=\Psi_{n} \theta_{n+1}(\eta)=$ $\theta_{n} \sigma^{\star}(\eta)=\theta_{n}(\eta)=\eta_{n}$. On the other hand, the previous paragraph shows that $\eta_{n}=\theta_{n}(\eta)=$ $\Phi_{n} \theta_{n+1}(\eta)=\Phi_{n}\left(\eta_{n+1}\right)$ for all $n \gg 0$. Thus $\left\{\eta_{n}\right\}$ is a sequence of points as in (ii).

Step 2: Conversely, suppose that we are given points $\eta_{n} \in W_{n}$ as in (ii), say for $n \geqslant n_{0}$. By Proposition 2.5, we can enlarge $n_{0}$ if necessary so that, for all $n \geqslant n_{0}$, both $\Psi_{n}^{-1}$ and $\Phi_{n}^{-1}$ are defined and hence local isomorphisms at $\eta_{n}$. Define $L=k\left(\eta_{n_{0}}\right)$ to be the residue field at $\eta_{n_{0}}$; this identification also induces a morphism $\rho_{n_{0}}$ : Spec $L \rightarrow W_{n_{0}}$ with $\operatorname{Im} \rho_{n_{0}}=\eta_{n_{0}}$. For $n \geqslant n_{0}$, we can inductively define $\rho_{n+1}=\Phi_{n}^{-1}\left(\rho_{n}\right)$; thus $\rho_{n+1}$ : $\operatorname{Spec} L \rightarrow W_{n+1}$ satisfies $\operatorname{Im} \rho_{n+1}=\eta_{n+1}$, and $\rho_{n}=\Phi_{n}\left(\rho_{n+1}\right)$ for all $n \geqslant n_{0}$. This means that there is an $L$-point module $M$ for $A$ which corresponds to the maps $\rho_{n}$.

Since $\Phi_{n}^{-1}$ and $\Psi_{n}^{-1}$ are defined at $\eta_{n}$ for $n \geqslant n_{0}$, our hypotheses imply that $\Psi_{n} \Phi_{n}^{-1}$ is a local isomorphism at $\eta_{n}$ with $\Psi_{n} \Phi_{n}^{-1}\left(\eta_{n}\right)=\eta_{n}$. Then there is a unique scheme automorphism $\widetilde{\tau}_{n}:$ Spec $L \rightarrow \operatorname{Spec} L$ satisfying $\rho_{n} \widetilde{\tau}_{n}=\Psi_{n} \Phi_{n}^{-1} \rho_{n}=\Psi_{n} \rho_{n+1}$. We claim that the morphisms $\widetilde{\tau}_{n}$ are all equal to each other. Indeed, for $n \geqslant n_{0}$, Lemma 2.3(iii) implies that

$$
\rho_{n} \widetilde{\tau}_{n}=\Psi_{n} \rho_{n+1}=\Psi_{n} \Phi_{n+1} \rho_{n+2}=\Phi_{n} \Psi_{n+1} \rho_{n+2}=\Phi_{n} \rho_{n+1} \widetilde{\tau}_{n+1}=\rho_{n} \widetilde{\tau}_{n+1} .
$$

Thus $\widetilde{\tau}_{n}=\widetilde{\tau}_{n+1}=\widetilde{\tau}$, say.

By Lemma 2.3(i), the truncations $N=\Phi_{n}^{\prime}(M)=M_{\leqslant n+1} / M_{n+1}$ and $N^{\prime}=\Psi_{n}^{\prime}\left(M_{\leqslant n+1}\right)=$ $\left(M_{\leqslant n+1}[1]\right)_{\geqslant 0}$ of $M_{\leqslant n+1}$ correspond to the scheme morphisms $\Phi_{n} \rho_{n+1}=\rho_{n}$ and $\Psi_{n} \rho_{n+1}=$ $\rho_{n} \widetilde{\tau}$, respectively. By the functoriality of $F_{n}$, as discussed earlier in Section $2, N^{\prime} \cong L_{\tau} \otimes N$, where $\tau: L \rightarrow L$ is the morphism induced from $\widetilde{\tau}$. Thus $M[1]_{\geqslant 0} \cong L_{\tau} \otimes M$. Let $P=\operatorname{ann}_{A} M_{s}$ for some $0 \leqslant s$. As the $A$-module and $L$-module actions on $M$ commute, $P$ is also the annihilator of $\left(L_{\tau} \otimes M\right)_{s}=M_{s+1}$. Hence, by induction, $P=\operatorname{ann}_{A} M$. As $M$ is a point module, $P \neq A_{\geqslant 1}$.

We will show that $P$ is a completely prime ideal such that $A / P$ is birationally commutative, as in (i). Suppose first that $a \in A_{u}$ and $b \in A_{v}$ are homogeneous elements with $a, b \notin P$. Thus $M_{0} a \neq 0$ which, since $M_{u}$ is a one-dimensional $L$-module, implies that $M_{0} a=M_{u}$. Similarly, as $P=\operatorname{ann}_{A} M_{u}$, we find that $M_{0} a b=M_{u} b=M_{u v}$. Thus, $a b \notin P$ and $P$ is a completely prime ideal of $A$. Set $\bar{A}=A / P$ and write $Q=Q(\bar{A})=Q_{0}\left[t, t^{-1} ; \gamma\right]$, for some $\gamma \in \operatorname{Aut}\left(Q_{0}\right)$ and $t \in \bar{A}_{1}$. Given nonzero homogeneous elements $m \in M_{s}, a \in \bar{A}_{u}$ and $b \in \bar{A}_{v}$ with $u \geqslant v$ then, once again, $M_{s+u}=L m a=M_{s+u-v} b$ and so there exists $n \in M_{s+u-v}$ with $n b=m a$. Then $m \otimes a b^{-1}=$ $n \otimes 1$ in $M \otimes_{A} Q$, and so $M \cong M \otimes_{A} Q_{\geqslant 0}$ already has a right $Q_{\geqslant 0}$-module structure. As $Q_{0}$ is a division ring and its right action on $M$ commutes with that of $L$, there is an injection of rings $f: Q_{0} \hookrightarrow \operatorname{End}_{L} M_{0}=L$. Thus $Q_{0}$ is isomorphic to a subfield $K$ of $L$. In particular, $\bar{A}$ is birationally commutative.

Step 3: We need to show that the operations from steps 1 and 2 are mutual inverses. To begin, we assume that we have chosen a sequence $\left\{\eta_{n}\right\}$ as in (ii), and performed step 2 to construct a completely prime ideal $P$ as in (i). Keep all of the notation of that step.

As $L$ is a field, the right action of $Q_{0}$ on $M_{0}$ coincides with the left action of the field $K \subseteq L$. Thus, for any $0 \neq m \in M_{0}$ the $A_{L}$-module surjection $L \otimes_{k} Q_{\geqslant 0} \rightarrow M$ given by $\ell \otimes$ $q \mapsto \ell m q$ induces a graded surjective $A_{L}$-module homomorphism $\alpha: L \otimes_{K} Q_{\geqslant 0} \rightarrow M$. Since $Q_{\geqslant 0}=Q_{0}[t ; \gamma]$, each graded piece both of $L \otimes_{K} Q_{\geqslant 0}$ and of $M$ is isomorphic to $L$. Thus $\alpha$ is an isomorphism.

We now perform step 1 with the ideal $P$ to construct maps $\theta_{n}$ : Spec $K \rightarrow W_{n}$ corresponding to the $K$-point module $Q_{\geqslant 0}$. If $f^{\star}: \operatorname{Spec} L \rightarrow \operatorname{Spec} K$ is the morphism associated to the 
injection of fields $f: K \hookrightarrow L$ then the isomorphism $L_{f} \otimes Q_{\geqslant 0} \cong M$ implies that $\rho_{n}$ factors as $\theta_{n} f^{\star}=\rho_{n}$ for all $n \geqslant n_{0}$. Thus $\eta_{n}=\operatorname{Im}\left(\theta_{n}\right)$ for all $n \geqslant n_{0}$ and so performing step 2 followed by step 1 gives the identity operation.

This also proves the final assertion of the proposition. Indeed, as the morphism $\rho_{n_{0}}$ is defined by the identification $k\left(\eta_{n_{0}}\right)=L$ the factorization $\theta_{n_{0}} f^{\star}=\rho_{n_{0}}$ forces $f$ to be the equality $K=$ $L$. Thus step 2 maps the set of points $\left\{\eta_{n}\right\}$ to a completely prime ideal $P$ such that $A / P$ has function division ring $K=L=k\left(\eta_{n}\right)$ for $n \gg 0$.

Step 4: Conversely, suppose we have chosen an ideal $P$ as in (i) and performed step 1, to obtain a sequence of maps $\theta_{n}:$ Spec $K \rightarrow W_{n}$ corresponding to the point module $Q(A / P)_{\geqslant 0}=$ $K[t ; \sigma]$, together with points $\eta_{n}=\operatorname{Im}\left(\theta_{n}\right) \in W_{n}$ as in (ii). Then we may perform step 2 to construct a point module $M$. As in step 2, write $L=k\left(\eta_{n_{0}}\right)$ with associated morphisms $\rho_{n}$ : $\operatorname{Spec} L \rightarrow W_{n}$ for $n \geqslant n_{0}$. In this case $\theta_{n_{0}}$ factors as $\theta_{n_{0}}=\rho_{n_{0}} \tilde{f}$ for a unique morphism of schemes $\tilde{f}: \operatorname{Spec} K \rightarrow \operatorname{Spec} L$, with corresponding ring map $f: L \rightarrow K$. Thus $\Phi_{n, n_{0}} \theta_{n}=\theta_{n_{0}}=$ $\rho_{n_{0}} \widetilde{f}=\Phi_{n, n_{0}} \rho_{n} \widetilde{f}$ for all $n \geqslant n_{0}$. By the choice of $n_{0}, \Phi_{n, n_{0}}^{-1}$ is a local isomorphism at $\eta_{n_{0}}$ and so $\theta_{n}=\rho_{n} \widetilde{f}$ for all $n \geqslant n_{0}$. By functoriality this implies that $K_{f} \otimes M \cong Q(A / P)_{\geqslant 0}$ as $A_{K^{-}}$ modules, and hence that $\operatorname{ann}_{A} M=\operatorname{ann}_{A}\left(Q(A / P)_{\geqslant 0}\right)=P$. Thus performing step 1 followed by step 2 is also the identity operation.

One of the most important cases of Proposition 3.3 occurs when $P$ is the zero ideal.

Corollary 3.5. Let $A$ be a cg birationally commutative noetherian domain which is generated in degree one and has graded quotient ring $Q(A)=K\left[t, t^{-1} ; \sigma\right]$, for some $t \in A_{1}$ and $\sigma \in \operatorname{Aut}(K)$. Then there exists $n_{0} \in \mathbb{N}$ such that:

(i) there is a sequence of points $\left\{\eta_{n}=\Phi_{n}\left(\eta_{n+1}\right)=\Psi_{n}\left(\eta_{n+1}\right) \in W_{n}\right\}$ corresponding to the $K$-point module $Q(A)_{\geqslant 0}$ such that $k\left(\eta_{n}\right) \cong K$ for all $n \geqslant n_{0}$

(ii) if $Y_{n}$ denotes the closure of $\eta_{n}$ in $W_{n}$ then $Y_{n}$ is a projective integral scheme such that the morphisms $\phi_{n}=\left.\Phi_{n}\right|_{Y_{n}}$ and $\psi_{n}=\left.\Psi_{n}\right|_{Y_{n}}$ are birational (and hence surjective) maps for all $n \geqslant n_{0}$.

Proof. (i) Apply Proposition 2.5 to the ideal $P=0$. By step 1 of the proof, the sequence of points that this constructs does indeed correspond to $Q(A)_{\geqslant 0}$.

(ii) By Proposition 2.5, $\Phi_{n}^{-1}$ and $\Psi_{n}^{-1}$ will be defined locally at $\eta_{n}$ for all $n \gg 0$ and so $\phi_{n}$ and $\psi_{n}$ will be birational for such $n$. The rest follows from part (i).

Definition 3.6. Keep the hypotheses and notation of Corollary 3.5. The scheme $Y_{n}$ will be called the relevant subscheme of $W_{n}$ and the data $\left(Y_{n}, \phi_{n}, \psi_{n}\right)$ will be called the relevant point scheme data for $A$. In Proposition 3.8 we will show that $Y_{n}$ is actually an irreducible component of $W_{n}$, after which we will call $Y_{n}$ the relevant component of $W_{n}$. We will usually write $\theta_{n}$ : Spec $K \rightarrow Y_{n}$ for the morphism defined by $K \cong k\left(\eta_{n}\right)$. In the notation of (2.6), the restrictions of $\Phi_{m, n}$ and $\Psi_{m, n}$ to $Y_{m}$ will be written $\phi_{m, n}$, respectively, $\psi_{m, n}$.

We want to briefly explain why the schemes $Y_{n}$ are important. So let $A$ be a noetherian cg domain that is generated in degree one and birationally commutative, with $Q(A)=K\left[t, t^{-1} ; \sigma\right]$. If $A$ is strongly noetherian then, as we explained in Section 1.4, $A$ is isomorphic in high degree to a twisted homogeneous coordinate $\operatorname{ring} B=B(Y, \mathcal{L}, \sigma)$ where the scheme $Y$ equals $W_{n}$ for any $n \gg 0$. 
Now suppose that $A$ is merely noetherian. The aim of this paper is, of course, to prove that $A$ is a naïve blowup algebra $R\left(Y, Z_{\mathcal{I}}, \mathcal{L}, \sigma\right)$, again up to a finite-dimensional vector space, for some scheme $Y$. Since $K=k(Y)$, the scheme $Y$ will necessarily be integral. Unfortunately, $Y$ will typically not equal any $W_{n}$ or even be birational to some $W_{n}$ simply because the $W_{n}$ can have the wrong dimension or have multiple components (see Example 11.1 and Remark 11.2). On the other hand the relevant subscheme $Y_{n}$ is an irreducible component of $W_{n}$ for all $n \gg 0$ (see Proposition 3.8, below) and, fortunately, it also contains all the information we need to reconstruct $A$. Indeed, the eventual scheme $Y$ will be obtained by blowing down $Y_{n}$ for any large $n$.

We will need to consider how the schemes $W_{n}$ and $Y_{n}$ are affected by the passage to Veronese rings. Given a noetherian cg domain $A$ generated in degree one and $p \geqslant 1$, the $p$ th-Veronese ring $A^{(p)}=\bigoplus_{n=0}^{\infty} A_{n p}$ of $A$ is again a cg noetherian domain that is generated in degree one (see [7, Proposition 5.10]) and we write $\left(W_{n}^{(p)}, \Phi_{n}^{(p)}, \Psi_{n}^{(p)}\right)$ for its point scheme data. If $A$ is also birationally commutative with $Q(A) \cong K\left[t, t^{-1} ; \sigma\right]$ and relevant subschemes $Y_{n} \subseteq W_{n}$, then $A^{(p)}$ will again be birationally commutative (its graded quotient ring is $K\left[t^{p}, t^{-p} ; \sigma^{p}\right]$ ) and we let $\left(Y^{(p)}, \phi_{n}^{(p)}, \psi_{n}^{(p)}\right)$ denote the relevant point scheme data.

Lemma 3.7. Let $A$ be a cg birationally commutative noetherian domain that is generated in degree one and pick $p \geqslant 1$.

(i) For all $n \geqslant 0$ there exist closed embeddings $\rho_{n}: W_{n p} \rightarrow W_{n}^{(p)}$ satisfying $\Phi_{n-1}^{(p)} \rho_{n}=$ $\rho_{n-1} \Phi_{n p,(n-1) p}$ and $\Psi_{n-1}^{(p)} \rho_{n}=\rho_{n-1} \Psi_{n p,(n-1) p}$, in the notation of (2.6).

(ii) The morphism $\rho_{n}$ restricts to an isomorphism $Y_{n p} \stackrel{\sim}{\longrightarrow} Y_{n}^{(p)}$ for all $n \gg 0$.

Proof. (i) Let $C$ be any commutative $k$-algebra. If $M=\bigoplus_{i=0}^{n p} M_{i}$ is a truncated point module of length $n p+1$ over $A_{C}=A \otimes C$ then $M^{(p)}=\bigoplus_{i=0}^{n} M_{i p}$ is a truncated point module of length $n+1$ over $A_{C}^{(p)}$. Since the map $M \mapsto M^{(p)}$ is compatible with change of rings, we obtain a morphism of the representing schemes $\rho_{n}: W_{n p} \rightarrow W_{n}^{(p)}$. The given decompositions of $\Phi_{n-1}^{(p)} \rho_{n}$ and $\Psi_{n-1}^{(p)} \rho_{n}$ then follow from Lemma 2.3(i).

It remains to show that $\rho_{n}$ is a closed embedding. Write $A=T(V) / J$, for $V=A_{1}$; thus $A^{(p)} \cong T\left(V^{\otimes p}\right) / \bigoplus_{n=0}^{\infty} J_{n p}$. Under these presentations, $W_{n p}$ is the zero set $\mathcal{V}\left(J_{n p}\right) \subseteq \mathbb{P}\left(V^{*}\right)^{\times n p}$ while $W_{n}^{(p)}=\mathcal{V}\left(J_{n}^{(p)}\right) \subseteq \mathbb{P}\left(V^{* \otimes p}\right)^{\times n}$. The map $\rho_{n}$ is just the restriction to $W_{n p}$ of the Segre embedding $\mathbb{P}\left(V^{*}\right)^{\times n p}=\left(\mathbb{P}\left(V^{*}\right)^{\times p}\right)^{\times n} \longrightarrow \mathbb{P}\left(V^{* \otimes p}\right)^{\times n}$. As such, $\rho_{n}$ is a closed embedding.

(ii) If $Q(A)=K\left[t, t^{-1}, \sigma\right]$ then $Q\left(A^{(p)}\right)=K\left[t^{p}, t^{-p}, \sigma^{p}\right]$. The $K$-point module $Q(A)_{\geqslant 0}$ corresponds to a sequence of morphisms $\theta_{n}$ : Spec $K \rightarrow W_{n}$ for $n \geqslant 0$ and, similarly, $Q\left(A^{(p)}\right) \geqslant 0$ is a $K$-point module for $A^{(p)}$ corresponding to morphisms $\theta_{n}^{\prime}$ : Spec $K \rightarrow W_{n}^{(p)}$. Since $Q(A)_{\geqslant 0}^{(p)}=$ $Q\left(A^{(p)}\right)_{\geqslant 0}$, it follows from part (i) that $\rho_{n} \theta_{n p}=\theta_{n}^{\prime}$ for each $n$. Since $Y_{n p}$ and $Y_{n}^{(p)}$ are the closures of the images of $\theta_{n p}$ and $\theta_{n}^{\prime}$, respectively, it follows that $\rho_{n}\left(Y_{n p}\right)=Y_{n}^{(p)}$ for each $n$. As $\rho_{n}$ is also a closed embedding, it must restrict to an isomorphism $Y_{n p} \stackrel{\sim}{\longrightarrow} Y_{n}^{(p)}$.

To conclude the section, we will show that the relevant subschemes of a birationally commutative ring are irreducible components of the truncated point schemes. This result will not be used elsewhere in the paper.

Proposition 3.8. Let $A$ be a birationally commutative noetherian cg domain, with point scheme data $\left(W_{n}, \Phi_{n}, \Psi_{n}\right)$ and relevant subschemes $Y_{n} \subseteq W_{n}$. Then $Y_{n}$ is an irreducible component of $W_{n}$ for all $n \gg 0$. 
Proof. Keep the notation from Corollary 3.5 and Definition 3.6; in particular, $\eta_{n}$ is the generic point of $Y_{n}$ and $\Phi_{n}^{-1}$ and $\Psi_{n}^{-1}$ define local isomorphisms at $\eta_{n}$ for all $n \geqslant n_{0}$. For each such $n$, let $S_{n}$ be the set of irreducible components of $W_{n}$ which contain $Y_{n}$.

Suppose first that $\operatorname{dim} \Phi_{n}(Z)<\operatorname{dim} Z$ for some $Z \in S_{n+1}$ with $n \geqslant n_{0}$. Then for every closed point $z \in \Phi_{n}(Z)$, and in particular for $z \in \Phi_{n}\left(Y_{n+1}\right)=Y_{n}$, the fibre $\Phi_{n}^{-1}(z)$ has dimension at least 1. This contradicts the fact that $\Phi_{n}^{-1}$ defines a local isomorphism at $\eta_{n}$ and proves that $\operatorname{dim} \Phi_{n}(Z)=\operatorname{dim} Z$ for all $Z \in S_{n+1}$. Similarly, $\operatorname{dim} \Psi_{n}(Z)=\operatorname{dim} Z$ for all $Z \in S_{n+1}$. For $n \geqslant n_{0}$ let $d_{n}$ be the maximum dimension of the components in $S_{n}$. Since $\Phi_{n}(Z) \subseteq Z^{\prime}$ for some $Z^{\prime} \in S_{n}$, we have $d_{n+1} \leqslant d_{n}$ for $n \geqslant n_{0}$. Thus $d_{n}$ is constant for $n \gg 0$, say $d_{n}=d$ for $n \geqslant$ $n_{1} \geqslant n_{0}$.

For each $n \geqslant n_{1}$ let $T_{n} \subseteq S_{n}$ consist of those components of dimension $d$. Let $\Gamma_{n}$ be the set of generic points of the components in $T_{n}$. Then $\Phi_{n}\left(\Gamma_{n+1}\right) \subseteq \Gamma_{n}$ and similarly $\Psi_{n}\left(\Gamma_{n+1}\right) \subseteq \Gamma_{n}$ for all $n \geqslant n_{1}$. By Corollary 2.7, we may pick $n_{2} \geqslant n_{1}$ such that for $n \geqslant n_{2}$ both $\Phi_{n}$ and $\Psi_{n}$ give bijections from $\Gamma_{n+1}$ to $\Gamma_{n}$ and both $\Phi_{n}^{-1}$ and $\Psi_{n}^{-1}$ define local isomorphisms at each point of $\Gamma_{n}$. In particular, $\left|T_{n}\right|$ is a constant value $m$ for $n \geqslant n_{2}$. Now $\Phi_{n} \Psi_{n+1}=\Psi_{n} \Phi_{n+1}$ by Lemma 2.3(iii). Thus, for each $n \geqslant n_{2}$, we can label $\Gamma_{n}=\left\{\mu_{1, n}, \ldots, \mu_{m, n}\right\}$ in such a way that $\Phi_{n}\left(\mu_{i, n+1}\right)=\mu_{i, n}$ and $\Psi_{n}\left(\mu_{i, n+1}\right)=\mu_{\tau(i), n}$, where $\tau$ is a permutation in the symmetric group on $\{1,2, \ldots, m\}$, independent of the choice of $n$.

Set $p=|\tau|$ and write $A^{\prime}=A^{(p)}$, with point scheme data $\left(W_{n}^{\prime}, \Phi_{n}^{\prime}, \Psi_{n}^{\prime}\right)$ and relevant subschemes $Y_{n}^{\prime} \subseteq W_{n}^{\prime}$. Let $\rho_{n}: W_{n p} \rightarrow W_{n}^{\prime}$ be the closed embeddings of Lemma 3.7; thus $Y_{n}^{\prime}=\rho_{n}\left(Y_{n p}\right)$ by that lemma. If $\eta_{n}^{\prime}$ denotes the generic point of each $Y_{n}^{\prime}$, then $\Psi_{n}^{\prime}\left(\eta_{n+1}^{\prime}\right)=$ $\Phi_{n}^{\prime}\left(\eta_{n+1}^{\prime}\right)=\eta_{n}^{\prime}$ for all $n \gg 0$. On the other hand, if $\mu_{n}^{\prime}=\rho_{n}\left(\mu_{1, n p}\right)$, then Lemma 3.7 shows that $\Psi_{n}^{\prime}\left(\mu_{n+1}^{\prime}\right)=\Phi_{n}^{\prime}\left(\mu_{n+1}^{\prime}\right)=\mu_{n}^{\prime}$ for all $n \gg 0$. Applying Proposition 3.3 to the ring $A^{\prime}$ shows that the points $\left\{\eta_{n}^{\prime}\right\}$ correspond to the zero ideal while the $\left\{\mu_{n}^{\prime}\right\}$ correspond to some (possibly different) completely prime ideal $P$ of $A^{\prime}$.

We need to show that $P=0$. Note that $\eta_{n}^{\prime}$ is in the Zariski closure $Z_{n}^{\prime}=\overline{\mu_{n}^{\prime}}$ for all $n \geqslant n_{2}$. By Proposition 2.5, pick $n_{3} \geqslant n_{2}$ such that $\left(\Phi_{n}^{\prime}\right)^{-1}$ defines a local isomorphism both at $\eta_{n}^{\prime}$ and at $\mu_{n}^{\prime}$ for all $n \geqslant n_{3}$. Let $R$ be the local ring $R=\mathcal{O}_{Z_{n_{3}}^{\prime}, \eta_{n_{3}}^{\prime}}$ with field of fractions $L \cong k\left(\mu_{n_{3}}^{\prime}\right)$

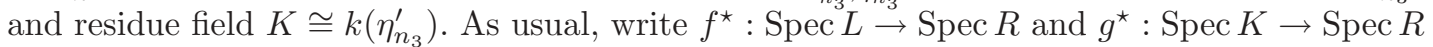
for the morphisms induced from the corresponding maps $f: R \rightarrow L$ and $g: R \rightarrow K$. Then we can define morphisms $\alpha_{n_{3}}$ : Spec $K \rightarrow W_{n}^{\prime}$ with image $\eta_{n}^{\prime}$, as well as $\beta_{n_{3}}$ : Spec $L \rightarrow W_{n}^{\prime}$ with image $\mu_{n}^{\prime}$, and $\gamma_{n_{3}}$ : Spec $R \rightarrow W_{n}^{\prime}$ in such a way that $\alpha_{n_{3}}=\gamma_{n_{3}} g^{\star}$ and $\beta_{n_{3}}=\gamma_{n_{3}} f^{\star}$. Since $\left(\Phi_{n, n_{3}}^{\prime}\right)^{-1}$ is defined as a morphism on $\operatorname{Im}\left(\gamma_{n_{3}}\right)$, we can inductively define maps $\gamma_{n+1}=$ $\left(\Phi_{n}^{\prime}\right)^{-1} \gamma_{n}$ :Spec $R \rightarrow W_{n+1}^{\prime}$ such that $\Phi_{n}^{\prime} \gamma_{n+1}=\gamma_{n}$ for all $n \geqslant n_{3}$. The morphisms $\alpha_{n}=\gamma_{n} g^{\star}$ and $\beta_{n}=\gamma_{n} f^{\star}$ then satisfy $\Phi_{n}^{\prime} \alpha_{n+1}=\alpha_{n}$ and $\Phi_{n}^{\prime} \beta_{n+1}=\beta_{n}$ for all $n \geqslant n_{3}$.

The sequences of maps $\left\{\alpha_{n}\right\},\left\{\beta_{n}\right\},\left\{\gamma_{n}\right\}$ for $n \geqslant n_{3}$ correspond to point modules $M, N$ and $Q$ over the rings $K, L$ and $R$, respectively, such that $K_{g} \otimes_{R} Q \cong M$ and $L_{f} \otimes_{R} Q \cong N$. It follows that $\operatorname{ann}_{A} N=\operatorname{ann}_{A} Q \subseteq \operatorname{ann}_{A} M$. By the correspondence of Proposition 3.3, this means that $P \subseteq(0)$, whence $P=0$. This forces $\mu_{n}^{\prime}=\eta_{n}^{\prime}$ for all $n \gg 0$ which, since each $\rho_{n}$ is a closed embedding, implies that $\mu_{1, n p}=\eta_{n}$ for each such $n$. Thus $Y_{n}$ is indeed a component of $W_{n}$.

\section{Contracted points}

Let $A$ be a noetherian cg domain that is birationally commutative. If the morphisms $\phi_{n}, \psi_{n}$ : $Y_{n+1} \rightarrow Y_{n}$ are isomorphisms for $n \gg 0$ then it is not hard to understand the structure of $A$ and, indeed, to prove that $A$ is a twisted homogeneous coordinate ring in large degree (see $[\mathbf{2 4}$, Theorem 4.4, Corollary 4.6]). To understand the general case, it will be useful to examine the points where the maps $\phi_{n}$ and $\psi_{n}$ are not isomorphisms, with particular emphasis on the points where they have infinite fibres, and this is what we do over the next four sections. Eventually, 
in Theorem 7.1, this information will be used to construct a surjective image of $Y_{n}$, for some $n \gg 0$, on which the birational map $\sigma=\psi_{n} \phi_{n}^{-1}$ induces an isomorphism.

To achieve this aim we will have to restrict our attention to surfaces and so, from now on, we will assume the following.

Assumptions 4.1. The algebra $A$ is a cg noetherian domain, generated in degree one, which is birationally commutative with graded quotient $\operatorname{ring} Q=K\left[t, t^{-1} ; \sigma\right]$, where $K$ is a field with tr. $\operatorname{deg} K / k=2$.

The points that interest us are described as follows.

Notation 4.2. Given an algebra $A$ as in (4.1), let $\left(Y_{n}, \phi_{n}, \psi_{n}\right)$ denote the relevant point scheme data from Definition 3.6. By Corollary 3.5, fix an integer $n_{0}$ such that $k\left(Y_{n}\right) \cong K$ and the morphisms $\phi_{n}, \psi_{n}$ are birational, for all $n \geqslant n_{0}$. For $n \geqslant n_{0}$, let $S_{n}^{-}$denote the set of closed points $y \in Y_{n}$ such that the fibre $\phi_{n}^{-1}(y)$ has dimension at least 1 . Similarly, let $S_{n}^{+}$be the set of points $y \in Y_{n}$ such that the fibre $\psi_{n}^{-1}(y)$ has dimension at least 1 . The points in $S_{n}^{-}$and $S_{n}^{+}$will be called contracted points. As $\operatorname{dim} Y_{n}=2$ it follows, for example, from $[\mathbf{1 4}$, Exercise II.3.22(d)], that $S_{n}^{-}, S_{n}^{+}$are finite sets.

EXAMPLE 4.3. In order to illustrate these concepts, we consider the example $A=$ $R\left(\mathbb{P}^{2}, Z, \mathcal{O}(1), \sigma\right)$ from Section 1.2 , where $Z$ is the single reduced point $c=(1: 1: 1)$ and $p, q$ are algebraically independent over the prime subfield of $k$. We will not prove the assertions we make about $A$ except to note that, at the level of sets, our description of the closed points of $Y_{n}$ follows from [20, Theorem 6.6]. To see this, note that, by Corollary 3.5(ii), each truncated point module $y_{n}$ corresponding to a point in $Y_{n}$ is a homomorphic image of a point module. Those point modules are then described by [20, Theorem 6.6]. The proof that we have the correct description of $Y_{n}$ as a scheme is more complicated and is omitted.

Write $r_{m}=\sigma^{1-m}(c)=\left(1: p^{1-m}: q^{1-m}\right)$ for $m \in \mathbb{Z}$. If $n$ is sufficiently large then $Y_{n}$ is the scheme obtained as the blowup $\gamma_{n}: Y_{n} \rightarrow \mathbb{P}^{2}$ of $\mathbb{P}^{2}$ at $Z_{n}=\left\{r_{1}, \ldots, r_{n}\right\}$. Write the resulting exceptional curves as $C_{i}^{(n)}=\gamma_{n}^{-1}\left(r_{i}\right)$ for $1 \leqslant i \leqslant n$ but, by a slight abuse of notation, set $r_{m}=\gamma^{-1}\left(r_{m}\right)$ for $m \notin[1, n]$. The maps $\phi_{n}$ and $\psi_{n}$ are easily described. The morphism $\phi_{n}$ : $Y_{n+1} \rightarrow Y_{n}$ is simply obtained by blowing up $r_{n+1}$ to obtain the new exceptional curve $C_{n+1}^{(n+1)}$. On the other hand, $\psi_{n}: Y_{n+1} \rightarrow Y_{n}$ is obtained by blowing up $r_{0}=c$ and then 'shifting by $\sigma$ '; thus $\psi_{n}^{-1}\left(r_{0}\right)=C_{1}^{(n)}$ while $\psi_{n}^{-1}\left(C_{j}^{(n)}\right)=C_{j+1}^{(n+1)}$ for $1 \leqslant j \leqslant n$.

We remark that the conventions from the previous paragraph are the ones that are most natural for naïve blowups (see, for example, [16]). However, the notation that is most useful for this paper is the opposite, thus $S_{n}^{-}=\left\{r_{n+1}\right\}$ and $S_{n}^{+}=\left\{r_{0}\right\}$.

The following result gives the most basic properties of the contracted points.

Lemma 4.4. Let $A$ satisfy (4.1) and keep the notation of (4.2). Then there exists $n_{1} \geqslant n_{0}$ such that:

(i) for all $p \geqslant n_{0}$ one has $\psi_{p}\left(S_{p+1}^{-}\right) \subseteq S_{p}^{-}$and $\phi_{p}\left(S_{p+1}^{+}\right) \subseteq S_{p}^{+}$;

(ii) for all $q>p \geqslant n_{1}$ the birational map $\psi_{q, p}^{-1}$ is defined at each $s \in S_{p}^{-}$, with $\psi_{q, p}^{-1}(s) \in S_{q}^{-}$. Similarly, $\phi_{q, p}^{-1}(t)$ is defined and belongs to $S_{q}^{+}$for each $t \in S_{p}^{+}$;

(iii) for all $p \geqslant n_{1}, S_{p}^{-} \cap S_{p}^{+}=\emptyset$ and the cardinalities $\left|S_{p}^{-}\right|$and $\left|S_{p}^{+}\right|$are constant. 
Proof. In parts (i) and (ii), it suffices by symmetry to prove the statements for $S_{p}^{-}$.

(i) Recall that we have a closed immersion $\iota_{p}: W_{p} \rightarrow \mathbb{P}^{\times p}$, and the morphisms $\Phi_{p}, \Psi_{p}$ : $W_{p+1} \rightarrow W_{p}$ are defined to be the restrictions of the respective projection maps $\pi_{1, p}, \pi_{2, p+1}$ : $\mathbb{P}^{\times(p+1)} \rightarrow \mathbb{P}^{\times p}$. Now let $y \in S_{p+1}^{-}$, say given by $y=\left(y_{1}, \ldots, y_{p+1}\right) \in \mathbb{P}^{\times(p+1)}$. By definition, $y$ has infinitely many preimages under $\phi_{p+1}$, say $z_{\alpha}=\left(y_{1}, \ldots, y_{p+1}, \alpha\right) \in Y_{p+2} \subseteq \mathbb{P}^{\times(p+2)}$, for some infinite set of $\alpha \in \mathbb{P}$. The points $w_{\alpha}=\psi_{p+1}\left(z_{\alpha}\right)=\left(y_{2}, \ldots, y_{p+1}, \alpha\right)$ are then distinct elements of $Y_{p+1}$, each of which satisfies $\phi_{p}\left(w_{\alpha}\right)=\left(y_{2}, y_{3}, \ldots, y_{p+1}\right)=\psi_{p}(y) \in Y_{p}$. Hence $\psi_{p}(y) \in S_{p}^{-}$.

(ii) The sets $S_{p}^{-}$are finite for $p \geqslant n_{0}$ and $\Psi_{p}\left(S_{p+1}^{-}\right) \subseteq S_{p}^{-}$by part (i). By Corollary 2.7 there exists $n_{1} \geqslant n_{0}$ such that, if $p \geqslant n_{1}$ then $\left|S_{p}^{-}\right|$is constant and $\Psi_{p}^{-1}$ is locally an isomorphism on $S_{p}^{-}$. For such $p$, the restriction $\psi_{p}^{-1}$ will still be a local isomorphism at $S_{p}^{-}$and so, by induction, the birational map $\psi_{q, p}^{-1}: Y_{p} \rightarrow Y_{q}$ is defined at $S_{p}^{-}$for all $q>p \geqslant n_{1}$.

(iii) Repeating the argument of part (ii) for the maps $\Phi_{n}$, we can choose a single $n_{1}$ so that the cardinalities $\left|S_{p}^{-}\right|$and $\left|S_{p}^{+}\right|$remain constant for $p \geqslant n_{1}$. Since $\psi_{p}^{-1}$ is defined on $S_{p}^{-}$, the map $\psi_{p}$ obviously cannot have a fibre of positive dimension over $S_{p}^{-}$. Thus $S_{p}^{-} \cap S_{p}^{+}=\emptyset$, as required.

By definition, the birational map $\phi_{p}^{-1}: Y_{p} \rightarrow Y_{p+1}$ is not defined at any point $y \in S_{p}^{-}$. Conversely, we would like to say that for $p \gg 0$, the domain of definition of $\phi_{p}^{-1}$ is exactly $Y_{p} \backslash S_{p}^{-}$. Although this holds for Example 4.3, in general it is too strong an assertion. However, something weaker is true; as we next show, the points where $\phi_{p}^{-1}$ is undefined are either contracted points themselves, or else lie on a curve which contracts at a later stage.

Lemma 4.5. Let $A$ satisfy (4.1) and keep the notation of (4.2). Fix $n_{1} \geqslant n_{0}$. Then there exists $n_{2} \geqslant n_{1}$ with the following properties.

(i) For some $m \geqslant n_{2}$, let $y \in Y_{m}$ be a closed point such that the map $\phi_{m}^{-1}$ is not defined at $y$. Then either $y \in S_{m}^{-}$, or else there exists $m>p \geqslant n_{1}$ and a curve $C \subset Y_{m}$ such that $y \in C$ and $\phi_{m, p}(C) \in S_{p}^{-}$.

(ii) Suppose that $\psi_{m}^{-1}$ is not defined at a closed point $y \in Y_{m}$ for some $m \geqslant n_{2}$. Then either $y \in S_{m}^{+}$, or else $y \in C \subset Y_{m}$ for some curve $C$ with $\psi_{m, p}(C) \in S_{n}^{+}$for some $m>p \geqslant n_{1}$.

Proof. As usual, we prove only part (i); the proof of part (ii) is analogous.

The main idea of the proof is to study the morphisms $\phi_{m, n}$ through Stein factorizations, as described in [14, Corollary III.11.5], and we start with the relevant notation. Choose integers $m>n \geqslant n_{1}$ and, for ease of notation, set $q=n_{1}$. The Stein factorization $\phi_{m, n}=g_{m, n} \circ h_{m, n}$ of the morphism $\phi_{m, n}: Y_{m} \rightarrow Y_{n}$ has the following properties: $h_{m, n}: Y_{m} \rightarrow Z_{m, n}$ is a projective morphism to a scheme defined by $Z_{m, n}=\operatorname{Spec}\left(\phi_{m, n}\right)_{*} \mathcal{O}_{Y_{m}}$, while the morphism $g_{m, n}: Z_{m, n} \rightarrow$ $Y_{n}$ is finite. We remark that $h_{m, n}$ necessarily has connected fibres and, since $\phi_{m, n}$ is birational, the maps $g_{m, n}$ and $h_{m, n}$ must also be birational.

By Corollary $3.5 \phi_{m, q}: Y_{m} \rightarrow Y_{q}$ is a birational surjective morphism for all $m \geqslant q$ and so we get an ascending chain of coherent sheaves of $\mathcal{O}_{Y_{q}}$-algebras,

$$
\mathcal{O}_{Y_{q}} \subseteq\left(\phi_{q+1, q}\right)_{*} \mathcal{O}_{Y_{q+1}} \subseteq \ldots \subseteq\left(\phi_{m, q}\right)_{*} \mathcal{O}_{Y_{m}} \subseteq \ldots
$$

and associated to this a chain of finite morphisms

$$
\cdots \stackrel{e_{m}}{\longrightarrow} Z_{m, q} \stackrel{e_{m-1}}{\longrightarrow} \cdots \stackrel{e_{q+1}}{\longrightarrow} Z_{q+1, q} \stackrel{e_{q}}{\longrightarrow} Z_{q}=Y_{q}
$$

where $g_{m, q}=e_{q} \circ e_{q+1} \circ \ldots \circ e_{m-1}$ is the finite morphism half of the Stein factorization of $\phi_{m, q}$. Choose a finite open affine cover $Y_{q}=\bigcup U_{\alpha}$ of $Y_{q}$. Then looking at the sections of (4.6) over any $U_{\alpha}$ gives an ascending chain of finite ring extensions, which must eventually stabilize by 
the finiteness of the integral closure [11, Theorem 4.14]. Thus (4.6) must also stabilize and so $e_{m}$ must be an isomorphism for all $m \gg q$, say for all $m \geqslant n_{2}$.

For $m>n_{2}$ we have a commutative square:

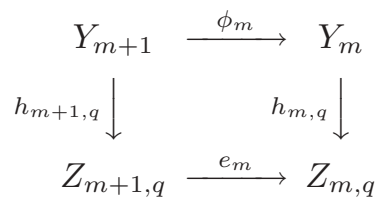

By assumption $e_{m}$ is an isomorphism and so the map $\theta=h_{m, q} \phi_{m}=e_{m} h_{m+1, q}: Y_{m+1} \rightarrow Z_{m, q}$ has connected fibres, since $h_{m+1, q}$ does. Let $y \in Y_{m}$ be a closed point where the birational map $\phi_{m}^{-1}$ is not defined and consider $z=h_{m, q}(y) \in Z_{m, q}$. If the birational map $\theta^{-1}$ were defined at $z$, then $\phi_{m}^{-1}=\theta^{-1} h_{m, q}$ would be defined at $y$, contradicting our assumption. So $\theta^{-1}$ is not defined at $z$.

Suppose now that the set-theoretic fibre $\theta^{-1}(z)$ is a single point $z^{\prime}$. By construction, $\theta=$ $e_{m} h_{m+1, q}$ is projective with $\theta_{*} \mathcal{O}_{Y_{m+1}}=\mathcal{O}_{Z_{m, q}}$. Thus Lemma (ii) can be applied to show that the birational map $\theta^{-1}$ must have been defined at $z$ after all, a contradiction. As $\theta$ has connected fibres, we conclude that the fibre $\theta^{-1}(z)$ must contain an irreducible curve $C$ such that $y \in$ $\phi_{m}(C)$. Since

$$
\phi_{m+1, q}=\phi_{m, q} \phi_{m}=g_{m, q} h_{m, q} \phi_{m}=g_{m, q} \theta
$$

we see that $\phi_{m+1, q}(C)=g_{m, q}(z)$ is a closed point in $Y_{q}$. Now let $p$ be the largest integer with $m \geqslant p \geqslant q$ for which $\phi_{m+1, p}(C)$ is a closed point $w \in Y_{p}$. Then $w \in S_{p}^{-}$and $\phi_{m, p}(y)=w$. If $p=m$, then $w=y \in S_{m}^{-}$; otherwise $m>p \geqslant q$ and $\phi_{m}(C)=D$ is a curve, containing $y$, for which $\phi_{m, p}(D)=w \in S_{p}^{-}$.

\section{The stable scheme and its birational map}

Throughout this section $A$ will satisfy Assumptions 4.1 and we keep the notation from Notation 4.2. In particular, $Q=Q(A)=K\left[t, t^{-1} ; \sigma\right]$ for some $\sigma \in \operatorname{Aut}_{k} K$. We are interested in the birational map that $\sigma$ induces on $Y_{n}$ for $n \gg 0$ and, for simplicity, we continue to write this as $\sigma: Y_{n} \rightarrow Y_{n}$. As we will see in this section, the birational map $\sigma$ has a number of special properties.

These properties are fairly technical but it is at least possible to indicate where the results are heading (see Proposition 6.4 and Theorem 7.1 for the formal statements). The basic idea is that we are aiming to prove that $Y_{n}$ is the blowup $\pi_{n}: Y_{n} \rightarrow \mathbb{X}_{n}$ of a scheme $\mathbb{X}_{n}$ such that (i) $\sigma$ is induced from an automorphism $\widetilde{\sigma}$ of $\mathbb{X}_{n}$ and (ii) the points that are blown up all lie on Zariski dense orbits of $\widetilde{\sigma}$. So, consider the set $V$ of closed points $y \in Y_{n}$ at which some power of $\sigma$ is undefined or not a local isomorphism. Under a minor extra hypothesis $V$ breaks into a finite union of sets $V=\bigcup V_{i}$ that are 'trying to be' infinite orbits of $\sigma$. Formally, within each $V_{i}$ there are two dense half orbits $V_{i}^{+}=\left\{y_{j, i}=\sigma^{j}\left(t_{i}\right): j \geqslant 0\right\}$ and $V_{i}^{-}=\left\{x_{j, i}=\sigma^{-j}\left(s_{i}\right): j \geqslant 0\right\}$ for some $s_{i}, t_{i} \in V_{i}$ to be determined. The remaining points in $V_{i}$, which essentially correspond to points on the exceptional divisor of $\pi_{n}$, are contained in a finite union of irreducible curves (and possibly some sporadic closed points) $C_{\ell}$ all of which are mapped to both $s_{i}$ and $t_{i}$ by suitable powers of $\sigma$; that is, there exist unique integers $a=a(\ell)>0$ and $b=b(\ell)<0$ such that $\sigma^{a}\left(C_{\ell}\right)=t_{i}$ and $\sigma^{b}\left(C_{\ell}\right)=s_{i}$.

The results mentioned in the previous paragraph only hold for sufficiently large values of $n$ and it is useful to more formally quantify 'sufficiently large' as follows. 
Definition 5.1. Define $n_{0}$ by Corollary 3.5. Then fix some $n_{1} \geqslant n_{0}$ such that the conclusions of Lemma 4.4 hold. Then take this $n_{1}$ in Lemma 4.5 and choose some $n_{2} \geqslant n_{1}$ satisfying the conclusions of that lemma. Finally, we call any $n>n_{2}$ a stable value of $n$.

Notation 5.2. Fix once and for all a stable value of $n$, and set $Y=Y_{n}$. Recall from Corollary 3.5 that for each $m \geqslant n$, the map $\theta_{m}$ : Spec $K \rightarrow W_{m}$ corresponding to the truncated $K$-point $\bigoplus_{i=0}^{m} Q_{i}$ induces an isomorphism $k\left(\eta_{m}\right) \rightarrow K$, where $\eta_{m}$ is the generic point of the relevant component $Y_{m}$. We identify $k(Y)=K$ through the map $\theta_{n}$ and, as before, write $\sigma: Y \rightarrow Y$ for the birational map induced by the automorphism $\sigma \in \operatorname{Aut}(K)$. Set $S^{+}=S_{n}^{+}$ and $S^{-}=S_{n}^{-}$for the contracted points, as defined in Notation 4.2.

To simplify notation, we often write $\phi_{m}=\phi$, for any $m \geqslant n_{0}$, where the subscript will be clear from the domain or range of $\phi$. Similar comments apply to $\psi$ and so, for example, for any $m \geqslant n_{0}$ the birational map $\psi_{m+s, m}^{-1}$ is now written $\psi^{-s}$. The next result, which shows how to interpret $\sigma^{m}: Y \rightarrow Y$ in terms of the morphisms $\phi$ and $\psi$, will be used frequently and usually without further comment.

Lemma 5.3. (i) As birational maps $Y_{p} \rightarrow Y_{p-m-\ell}$ one has $\phi^{\ell} \psi^{m}=\psi^{m} \phi^{\ell}$ for any $\ell, m \in \mathbb{Z}$ for which all the maps involved are birational (that is, as long as $p, p-m, p-\ell$ and $p-m-\ell$ are at least $n_{0}$ ).

(ii) For any $m \geqslant 0$, we have $\sigma^{m}=\psi^{m} \phi^{-m}$ and $\sigma^{-m}=\phi^{m} \psi^{-m}$ as birational maps $Y \rightarrow Y$.

Proof. (i) When $\ell, m \geqslant 0$ this follows from Lemma 2.3(iii). When $\ell$ or $m$ is negative, multiply through by the appropriate (birational) inverse map.

(ii) Recall from step 1 of Proposition 3.3 that $\theta_{n}=\Phi_{n} \theta_{n+1}$ and $\theta_{n} \sigma^{\star}=\Psi_{n} \theta_{n+1}$, where $\theta_{m}:$ Spec $K \rightarrow W_{m}$ is defined by Notation 5.2 and $\sigma^{\star}: \operatorname{Spec} K \rightarrow \operatorname{Spec} K$ is the map of schemes associated to $\sigma \in \operatorname{Aut}(K)$. Restricting to $Y$ we find that $\psi \phi^{-1} \theta_{n}=\psi \theta_{n+1}=\theta_{n} \sigma^{\star}$, and it follows that $\psi \phi^{-1}$ is precisely the birational map $\sigma: Y \rightarrow Y$ induced by $\sigma \in \operatorname{Aut}(K)$. That $\sigma^{-1}=$ $\phi \psi^{-1}$ follows immediately, and the results for all $m \geqslant 1$ follow by induction using part (i).

The rest of this section is devoted to proving successively stronger properties of $S^{+}$and $S^{-}$, and of certain special extremal subsets thereof, for our fixed stable value of $n$. The properties of $\left(S^{+}, \sigma\right)$ and $\left(S^{-}, \sigma^{-1}\right)$ are analogous and so we will first consider $\left(S^{+}, \sigma\right)$, referring the reader to Remark 5.9 for $\left(S^{-}, \sigma^{-1}\right)$. We begin by studying points $\mu \in Y$ which are fixed by some power $\sigma^{n}$ and show that they do not interfere with $S^{+}$.

Lemma 5.4. Let $\mu \in Y$ be a point such that $\sigma^{p}$ is defined at $\mu$ and $\sigma^{p}(\mu)=\mu$ for some $p>0$.

(i) If $\mu$ is a closed point of $Y$ then $\mu \notin S^{+}$.

(ii) If $\mu$ is the generic point of an irreducible curve $C$, then $C \cap S^{+}=\emptyset$.

Proof. The proof is by contradiction, so we assume that there is a closed point $z \in S^{+}$ where either $z=\mu$ in case (i), or $z \in C$ in case (ii). By Lemma 4.4(ii), $\phi^{-s}$ is defined at $z$ for any $s \geqslant 1$ and then $\phi^{-s}(z) \in S_{s+n}^{+}$. In either case the birational map $\phi^{-s}$ is also defined at $\mu_{n}=\mu$ for $s \geqslant 1$, so we can define $\mu_{r}=\phi^{n-r}(\mu)=\phi^{-1}\left(\mu_{r-1}\right) \in Y_{r}$ for all $r n$.

We claim that $\psi^{p}\left(\mu_{r+p}\right)=\mu_{r}$ for all $r \geqslant n$. When $r=n$ this is just the observation that $\psi^{p}\left(\mu_{n+p}\right)=\psi^{p} \phi^{-p}(\mu)=\sigma^{p}(\mu)=\mu$. We then prove it for all $r \geqslant n$ by induction: assuming the 
result is true for some $r \geqslant n$, then

$$
\psi^{p}\left(\mu_{r+1+p}\right)=\psi^{p} \phi^{-1}\left(\mu_{r+p}\right)=\phi^{-1} \psi^{p}\left(\mu_{r+p}\right)=\phi^{-1}\left(\mu_{r}\right)=\mu_{r+1}
$$

and so the result holds for $r+1$.

Consider the Veronese ring $A^{\prime}=A^{(p)}$ of $A$ with point scheme data $\left(W_{m}^{\prime}, \Phi_{m}^{\prime}, \Psi_{m}^{\prime}\right)$ and relevant point scheme data $\left(Y_{m}^{\prime}, \phi_{m}^{\prime}, \psi_{m}^{\prime}\right)$. By Lemma 3.7(ii), there is an isomorphism $\rho_{p}$ : $Y_{p m} \rightarrow Y_{m}^{\prime}$ for each $m \gg 0$ and we write $\mu_{m}^{\prime}=\rho_{m}\left(\mu_{m p}\right)$ for such $m$. It follows from the previous paragraph and Lemma 3.7(i) that $\psi^{\prime}\left(\mu_{m+1}^{\prime}\right)=\mu_{m}^{\prime}=\phi^{\prime}\left(\mu_{m+1}^{\prime}\right)$ for all such $m$. By Proposition 3.3, there is then a completely prime ideal $P$ of $A^{\prime}$ corresponding to this sequence of elements such that $\widehat{A}=A^{\prime} / P$ is birationally commutative, with function division ring $L \cong k\left(\mu_{m}^{\prime}\right)$ for $m \gg 0$.

Now consider the point scheme data $\left(\widehat{W}_{m}, \widehat{\Phi}_{m}, \widehat{\Psi}_{m}\right)$ for $\widehat{A}$. By Lemma 3.1, $\widehat{W}_{m}$ is a closed subscheme of $W_{m}^{\prime}$ for each $m$, and by construction $\mu_{m}^{\prime} \in \widehat{W}_{m}$ for $m \gg 0$. By assumption $L$ is a field of transcendence degree at most 1 over $k$, so [3, Theorem 0.1(ii)] implies that the GelfandKirillov dimension of $\widehat{A}$ satisfies GKdim $\widehat{A} \leqslant 2$. By [2, Theorem 4.24] $\widehat{A}$ is strongly noetherian and hence, by [8, Theorem E4.4] and Lemma 2.3(ii), the maps $\widehat{\Phi}_{m}$ and $\widehat{\Psi}_{m}$ are isomorphisms for all $m \gg 0$. Lemma 3.1 therefore implies that the fibre $\left(\Psi^{\prime}\right)^{-1}(y)$ is a singleton for all $y \in \widehat{W}_{m}$ and all $m \gg 0$.

Recall that $z=\mu$ in case (i), respectively, $z \in C$ in case (ii), and set $z_{p m}=\phi^{-p m+n}(z) \in Y_{p m}$ for all $m \gg 0$, so $z_{p m}$ is in the closure of $\mu_{p m}$. Then $z_{m}^{\prime}=\rho_{m}\left(z_{p m}\right)$ is in the closure of $\mu_{m}^{\prime}$, whence $z_{m}^{\prime} \in \widehat{W}_{m}$. By the argument above, the fibre $\left(\Psi^{\prime}\right)^{-1}\left(z_{m}^{\prime}\right)$ is a singleton for all $m \gg 0$. On the other hand, since $z_{p m} \in S_{p m}^{+}$, we can find an irreducible curve $D_{p m+p} \subset Y_{p m+m}$ such that $\psi^{p}\left(D_{p m+p}\right)=z_{p m}$. Then Lemma 3.7 implies that $\rho_{m+1}\left(D_{p m+p}\right)$ is a curve in $W_{m+1}^{\prime}$ that contracts to $z_{m}^{\prime}$ under $\Psi^{\prime}$; a contradiction.

Next, we reinterpret Lemmas 4.4 and 4.5 to see how the contracted points $S^{+}$are related to the points where $\sigma^{-1}$ is undefined.

Lemma 5.5. (i) Let $y \in S^{+} \subseteq Y$. Then $\sigma^{-1}$ is not defined at $y$, but $\sigma^{d}$ is defined at $y$ for all $d \geqslant 0$.

(ii) Conversely, let $y \in Y$ be a closed point where either $\psi^{-1}$ or $\sigma^{-1}$ is not defined. Then there exists $e \geqslant 0$ such that $\sigma^{e}(y)$ is defined and belongs to $S^{+}$.

Proof. (i) Since $y \in S^{+}$there exists, by definition, an irreducible curve $D \subset Y_{n+1}$ such that $\psi(D)=y$. Suppose that $\phi(D)=z$ is also a closed point; thus $z \in S^{-}$. By Lemma 4.4(i),

$$
w=\phi(y)=\phi \psi(D)=\psi \phi(D)=\psi(z) \in S_{n-1}^{+} \cap S_{n-1}^{-},
$$

contradicting Lemma 4.4(iii). This contradiction implies that $\phi(D)=C$ is a curve, and $w=$ $\psi(C)=\phi \psi(D)=\phi(y)$ is a closed point in $S_{n-1}^{+}$. By Lemma 4.4(ii), $\phi^{-1}$ is defined at $w$ and so $\sigma=\phi^{-1} \psi$ is defined at every point of $C$. Since $\sigma$ contracts $C$ to $y, \sigma^{-1}$ is not defined at $y$. On the other hand, by Lemma 4.4(ii) again, $\phi^{-d}(y)$ is defined for all $d \geqslant 0$ and so $\sigma^{d}(y)=\psi^{d} \phi^{-d}(y)$ is defined for all $d \geqslant 0$.

(ii) As $\sigma^{-1}=\phi \psi^{-1}$, if $\sigma^{-1}$ is not defined at $y$, then the birational map $\psi^{-1}$ cannot be defined at $y$. Thus we need only consider the case that $\psi^{-1}$ is not defined at $y$. Since $n$ is a stable value, Lemma 4.5(ii) implies that either $y \in S^{+}$or $y \in C \subset Y$ for some irreducible curve $C$ with the property that $z=\psi_{n, p}(C) \in S_{p}^{+}$for some $n>p \geqslant n_{1}$. In the former case, we can take $e=0$; in the latter case, Lemma 4.4 implies that $\phi_{n, p}^{-1}(z)$ is defined and in $S^{+}$, so taking $e=n-p$ we have $\sigma^{e}(y)=\psi^{e} \phi^{-e}(y)=\phi^{-e} \psi^{e}(y)=\phi^{-e}(z) \in S^{+}$. 
It can happen that some power $\sigma^{m}$ of $\sigma$ sends a point $y \in S^{+}$to another point in $S^{+}$but, as we show next, this cannot happen for $m \gg 0$. The transition between these two possibilities will be fundamental and so we define the extremal elements of $S^{+}$to be

$$
S_{\infty}^{+}=\left\{y \in S^{+}: \forall d \geqslant 1, \quad \sigma^{d}(y) \notin S^{+}\right\} .
$$

This set will turn out to be the set of distinguished elements $S_{\infty}^{+}=\left\{t_{i}\right\}$ mentioned in the introduction to this section. The dual set $S_{\infty}^{-}=\left\{s_{j}\right\}$ will be defined after Remark 5.9.

\section{Lemma 5.7. If $y \in S^{+}$then $\sigma^{e}(y) \in S_{\infty}^{+}$for a unique integer $e$, necessarily with $e \geqslant 0$.}

Proof. Let $y_{0}=y \in S^{+}$. By Lemma 5.5(i), $\sigma^{d}\left(y_{0}\right)$ is defined for all $d \geqslant 0$. If $y_{0} \notin S_{\infty}^{+}$, then there exists $d_{0}>0$ such that $\sigma^{d_{0}}\left(y_{0}\right)=y_{1} \in S^{+}$. Similarly, $\sigma^{d}\left(y_{1}\right)$ is defined for all $d \geqslant 0$ and so either $y_{1} \in S_{\infty}^{+}$or there is $d_{1}>0$ with $y_{2}=\sigma^{d_{1}}\left(y_{1}\right)=y_{2} \in S^{+}$. If this process continues forever, we will produce an infinite sequence of points $y_{0}, y_{1}, y_{2}, \ldots$ in the finite set $S^{+}$and so $y_{i}=$ $y_{j}$ for some $j>i$. But then $\sigma^{d}\left(y_{i}\right)=y_{j}=y_{i}$ for $d=d_{i}+d_{i+1}+\ldots+d_{j-1}>0$, contradicting Lemma 5.4(i). Thus the process is finite and $\sigma^{e}\left(y_{0}\right) \in S_{\infty}^{+}$for some $e \geqslant 0$.

If $\sigma^{e_{1}}\left(y_{0}\right)=w \in S_{\infty}^{+}$and $\sigma^{e_{2}}\left(y_{0}\right)=z \in S_{\infty}^{+}$for some (possibly negative) integers $e_{2}>e_{1}$, then Lemma 5.5(i) implies that $\sigma^{e_{2}-e_{1}}(w)=z$, contradicting the definition of $S_{\infty}^{+}$. So $e$ is unique.

We next study the special properties of the points $S_{\infty}^{+}$. The intuitive idea is that these points mark the boundary of bad behaviour of $\sigma$, in the sense that for $y \in S_{\infty}^{+}$the half-orbit $\left\{\sigma^{d}(y): d \geqslant 0\right\}$ behaves just like the half-orbit of an automorphism.

Proposition 5.8. (i) Let $y \in S_{\infty}^{+}$. Then $\sigma^{e}$ is defined and a local isomorphism at $\sigma^{d}(y)$ for all $e, d \geqslant 0$.

(ii) Let $y \in S^{+}$. The set of points $\left\{\sigma^{d}(y): d \geqslant 0\right\}$ is Zariski dense in $Y$, and each of these points is nonsingular.

Proof. (i) By Lemma 5.5(i), $y_{d}=\sigma^{d}(y)$ is defined for each $d \geqslant 0$. If $\sigma^{-1}$ is undefined at $y_{d}$ for some $d \geqslant 1$ then Lemma 5.5(ii) implies that $\sigma^{e}\left(y_{d}\right)=y_{d+e} \in S^{+}$for some $e \geqslant 0$, contradicting the definition of $S_{\infty}^{+}$. Thus $\sigma^{-1}$ is defined at each $y_{d}$ with $d \geqslant 1$ and so, by induction, $\sigma^{-d}$ is defined at $y_{d}$ for $d \geqslant 1$. Thus $\sigma^{-d}\left(y_{d}\right)=y$ and $\sigma^{d}$ is a local isomorphism at $y$. In addition, $\sigma=\sigma^{d+1} \sigma^{-d}$ is defined at $y_{d}$ for all $d \geqslant 0$, with $\sigma\left(y_{d}\right)=y_{d+1}$. Finally, $\sigma^{e}$ is a local isomorphism at every point $y_{d}$ with $d \geqslant 0$, since the inverse map $\sigma^{-e}$ is defined at $y_{d+e}$.

(ii) Given $y \in S_{\infty}^{+}$, the half orbit $P=\left\{y_{d}=\sigma^{d}(y): d \geqslant 0\right\}$ is defined by part (i). Suppose first that $y_{i}=y_{j}$ for some $j>i \geqslant 0$. By part (i), $\sigma^{-i}$ is defined at both $y_{i}$ and $y_{j}$, and so $y=\sigma^{-i}\left(y_{i}\right)=\sigma^{-i}\left(y_{j}\right)=y_{j-i}$. Then $\sigma^{j-i}(y)=y_{j-i}=y$, contradicting Lemma 5.4(i). So $y_{i} \neq$ $y_{j}$ for $j \neq i$ and $P$ is infinite.

Suppose that the Zariski closure $\bar{P}$ of $P$ is a closed subset of $Y$ of dimension 1 . Then $\bar{P}$ is a finite union $\bar{P}=C_{1} \cup \ldots \cup C_{e} \cup D$ of irreducible curves $C_{i}$ together with a (possibly empty) finite set of closed points $D$. Each $C_{i}$ contains infinitely many of the $y_{j}$, and since both $\sigma$ and $\sigma^{-1}$ are defined at every closed point in $P \backslash\left\{y_{0}\right\}$, both $\sigma$ and $\sigma^{-1}$ are defined at the generic point $\mu_{i}$ of $C_{i}$ for each $i$. In particular, $\sigma$ and $\sigma^{-1}$ permute the set $\left\{\mu_{1}, \ldots, \mu_{e}\right\}$. Since $D$ is finite, if there exists $y_{d} \in D$, then $\sigma^{p}\left(y_{d}\right) \in C_{j}$ for some $j$ and some $p>0$, which forces $y_{d} \in \sigma^{-p}\left(C_{j}\right) \subseteq \bigcup C_{i}$, a contradiction. Thus $D=\emptyset$ and $y \in C_{\ell}$ for some $\ell$. But $\sigma^{e !}\left(\mu_{\ell}\right)=\mu_{\ell}$ and so Lemma 5.4(ii) implies that $C_{\ell} \cap S^{+}=\emptyset$, a contradiction. The only remaining possibility is that $P$ is dense in $Y$. 
Finally, since the singular locus of $Y$ is a proper closed subset and $P$ is dense, some point of $P$ is nonsingular. Since $\sigma$ is a local isomorphism at each $y_{d}$ with $d \geqslant 0$, the local rings $\mathcal{O}_{Y, y_{d}}$ are isomorphic for all $d \geqslant 0$ and hence every point $y_{d}$ is nonsingular.

REMARK 5.9. Recall from Lemma 2.3(ii) that working with left modules in place of right modules interchanges the rôles of $\phi$ and $\psi$, and hence it also interchanges the rôles of $\sigma$ and $\sigma^{-1}$. This therefore provides analogues for $\left(S^{-}, \sigma^{-1}\right)$ of the results just proved for $\left(S^{+}, \sigma\right)$; formally, by combining Lemma 2.3(ii) with the left-hand versions of Lemmas 5.5, 5.7 and Proposition 5.8, one obtains the following results.

(i) Let $y \in Y$ be a closed point where either $\sigma$ or $\phi^{-1}$ is undefined. Then $\sigma^{e}(y)$ is defined and in $S^{-}$for some $e \leqslant 0$.

By analogy with the definition of $S^{+}$in (5.6), we let $S_{\infty}^{-}=\left\{y \in S^{-}: \forall n \geqslant 1, \sigma^{-n}(y) \notin S^{-}\right\}$ denote the extremal elements of $S^{-}$.

(ii) If $y \in S^{-}$then $\sigma$ is not defined at $y$. However $\sigma^{e}(y)$ is defined for all $e \leqslant 0$ and $\sigma^{e}(y) \in$ $S_{\infty}^{-}$for a unique integer $e$, necessarily with $e \leqslant 0$.

(iii) Let $y \in S_{\infty}^{-}$. Then $\sigma^{e}$ is defined and a local isomorphism at $\sigma^{d}(y)$, for all $d, e \leqslant 0$. The points $\sigma^{d}(y)$ are nonsingular for $d \leqslant 0$.

\section{Birationally geometric algebras}

We continue to suppose that $A$ satisfies Assumptions 4.1 and let $Y=Y_{n}$ be the relevant component of the truncated point scheme, for some fixed stable value of $n$. If we want to show that $A$ is a naïve blowup algebra, then we first have to contract $Y$ to a second variety $\mathbb{X}$ where $\sigma$ becomes a (biregular) automorphism. This is not possible under the present assumptions on $Y$. However, an obviously necessary condition for the existence of $\mathbb{X}$ is for $\sigma$ to be represented by an automorphism of some projective model $Z$ of $K=k(Y)$. If we make this extra assumption then $\mathbb{X}$ does exist (see Theorem 7.1) and in this section we set the scene by examining the more elementary consequences of this hypothesis.

We begin with the formal definition.

Definition 6.1. Let $B$ be a cg algebra which is a birationally commutative domain, with graded quotient ring $Q(B) \cong K\left[t, t^{-1} ; \sigma\right]$. Then $B$ is called birationally geometric if there is a projective integral scheme $Z$ with $k(Z)=K$ and an automorphism $\tau \in \operatorname{Aut}(Z)$ inducing $\sigma$. If moreover $Z$ is a surface then we say that $B$ is birationally 2-geometric.

If $B$ is a cg domain with $Q(B) \cong K\left[t, t^{-1} ; \sigma\right]$ where $K$ is a finitely generated field extension with tr. $\operatorname{deg} K / k=1$, then $B$ is automatically birationally geometric, take $Z$ to be the unique nonsingular model of $K$. The surface case is more subtle, but by the following lemma one can at least assume in the definition that the surface is nonsingular.

Lemma 6.2. Let $B$ be birationally 2-geometric, with $Q(B)=K\left[t, t^{-1} ; \sigma\right]$. Then there exists a nonsingular surface $Z$ with $k(Z)=K$ and $\tau \in \operatorname{Aut}(Z)$ inducing $\sigma$.

Proof. By hypothesis, there is some projective surface $Z^{\prime}$ with $K=k\left(Z^{\prime}\right)$ and $\tau^{\prime} \in \operatorname{Aut}\left(Z^{\prime}\right)$ inducing $\sigma$. Now take a resolution of singularities $\pi: Z \rightarrow Z^{\prime}$ following, say, [17, Remark B, p. 155]) and check that $\tau^{\prime}$ lifts to an automorphism $\tau$ of $Z$. 
REMARK 6.3. Keep the hypotheses from the lemma. If $K$ is not the function field of a ruled surface then there exists a unique minimal nonsingular model $Z$ of $K$ and so $\sigma$ is necessarily induced by an automorphism of $Z$ (see [10, Proposition 7.5]). On the other hand, if $K$ is the function field of a ruled surface, then there do exist automorphisms $\sigma: K \rightarrow K$ such that $\sigma$ is not induced by an automorphism of any projective model $Z$ of $K$. For example, if $K=k(u, v)$ is purely transcendental, then the automorphism $\sigma$ defined by $\sigma(u)=u$ and $\sigma(v)=u v$ is one such example. Further examples are constructed in [10, Remark 7.3].

For the rest of this section, we will assume that $A$ is a birationally 2-geometric cg domain that is generated in degree one, with graded quotient $\operatorname{ring} Q=K\left[t, t^{-1} ; \sigma\right]$. We continue to use the terminology from Notation 5.2; in particular we fix $Y=Y_{n}$ for some stable value of $n$ and note that $k(Y)=K$ with induced birational map $\sigma: Y \rightarrow Y$. In Section 5, we proved various special properties for the contracted points $S_{\infty}^{+}$, and by symmetry, for the points in $S_{\infty}^{-}$. The main application of the birationally 2-geometric hypothesis is to show that these two sets are closely interrelated.

Proposition 6.4. Let $A$ be a birationally 2-geometric cg domain that is generated in degree one and assume that $S_{\infty}^{-} \neq \emptyset$; say $S_{\infty}^{-}=\left\{s_{1}, \ldots, s_{N}\right\}$. Then $S_{\infty}^{+}$may be uniquely written as $S_{\infty}^{+}=\left\{t_{1}, \ldots, t_{N}\right\}$ so that, for each $i$, there exists a unique $d_{i} \geqslant 2$ with $\sigma^{d_{i}}\left(s_{i}\right)=t_{i}$ and $\sigma^{-d_{i}}\left(t_{i}\right)=s_{i}$. Moreover, $\sigma^{d_{i}}$ is a local isomorphism at $s_{i}$.

REMARK 6.5. Note that, by Proposition 6.4 and Remark 5.9, $S_{\infty}^{-} \neq \emptyset$ if and only if $S_{\infty}^{+} \neq \emptyset$. Moreover, by Remark 5.9 and Lemmas 5.5(i,ii) and 5.7, $S_{\infty}^{+}=\emptyset$ if and only if $\sigma$ is an automorphism of $Y$.

Proof. Fix $y \in S_{\infty}^{+}$. By Lemma 5.8, the set $P=\left\{y_{m}=\sigma^{m}(y): m \geqslant 0\right\}$ is defined. Moreover, $\sigma$ is defined and a local isomorphism at each point of $P$, and $P$ is a dense set in $Y$ consisting of nonsingular points. In particular $P$ is infinite and so $y_{i} \neq y_{j}$ for $i \neq j$. Let $g: \widetilde{Y} \rightarrow Y$ be the normalization of $Y$ and note that $g^{-1}$ defines an isomorphism locally at each nonsingular point of $Y$, in particular at each point of $P$. Write $\widetilde{P}=g_{\widetilde{Y}}^{-1}(P)=\left\{\widetilde{y}_{m} \equiv g^{-1}\left(y_{m}\right)\right\}$ and $\widetilde{\sigma}=g^{-1} \sigma g$ : $\widetilde{Y} \rightarrow \widetilde{Y}$ for the induced birational map. Clearly $\widetilde{P}$ is dense in $\widetilde{Y}$, and $\widetilde{y}_{i} \neq \widetilde{y}_{j}$ for $i \neq j$. By Lemma 6.2, there exists a nonsingular model $Z$ of $K$ and a birational map $f: Z \rightarrow \widetilde{Y}$ such that $\tau=f^{-1} \widetilde{\sigma} f$ is an automorphism of $Z$.

Since $f$ is a birational map between normal surfaces, there are at most finitely many points where $f$ fails to be defined [14, Lemma V.5.1], say $G \subseteq Z$, and finitely many points, say $H \subseteq \widetilde{Y}$, where $f^{-1}$ is undefined. Since $H$ can contain only finitely many of the $\widetilde{y}_{i}$, there exists $m_{0} \geqslant 0$ such that $f^{-1}$ is defined at $\widetilde{y}_{m}$ for $m \geqslant m_{0}$. Set $z_{m}=f^{-1}\left(\widetilde{y}_{m}\right)$ for $m \geqslant m_{0}$ and note that $\left\{z_{m}: m \geqslant m_{0}\right\}$ is dense in $Z$. We compute that

$$
\tau\left(z_{m}\right)=\tau f^{-1}\left(\widetilde{y}_{m}\right)=f^{-1} g^{-1} \sigma g\left(\widetilde{y}_{m}\right)=f^{-1} g^{-1} \sigma\left(y_{m}\right)=f^{-1} g^{-1}\left(y_{m+1}\right)=z_{m+1}
$$

for all $m \geqslant m_{0}$. Thus $\left\{z_{m}: m \geqslant m_{0}\right\}$ extends to a full dense $\tau$-orbit $\left\{z_{m}=\tau^{m-m_{0}}\left(z_{m_{0}}\right): m \in\right.$ $\mathbb{Z}\}$ and again, $z_{i} \neq z_{j}$ for $i \neq j$.

Recall that $y=y_{0} \in S_{\infty}^{+}$, and so $\sigma^{-1}$ is not defined at $y$ by Lemma 5.5(i). On the other hand, since $G$ is finite we must have $z_{m} \notin G$ for all $m \ll 0$. Hence

$$
\sigma^{m}(y)=\sigma^{m-m_{0}}\left(y_{m_{0}}\right)=g f \tau^{m-m_{0}} f^{-1} g^{-1}\left(y_{m_{0}}\right)=g f \tau^{m-m_{0}}\left(z_{m_{0}}\right)=g f\left(z_{m}\right)
$$

is defined for all $m \ll 0$. Let $a<0$ be the largest negative integer for which $\sigma^{a}(y)$ is defined, so $a \leqslant-2$. Since $\sigma^{a+1}$ is not defined at $y$, the map $\sigma$ is not defined at $\sigma^{a}(y)$. By Remark 5.9(i) there exists $b \leqslant 0$ such that $\sigma^{b}\left(\sigma^{a}(y)\right)=\sigma^{a+b}(y)$ is defined and belongs to $S^{-}$, and then by 
Remark 5.9(ii) there exists an integer $c \leqslant 0$ with $\sigma^{c} \sigma^{a+b}(y)=\sigma^{a+b+c}(y) \in S_{\infty}^{-}$. Set $e=a+$ $b+c \leqslant-2$. If $e^{\prime}$ is a second integer with $\sigma^{e^{\prime}}(y) \in S_{\infty}^{-}$, say with $e^{\prime}>e$, then Remark 5.9(iii) implies that $\sigma^{e-e^{\prime}}$ is defined at $\sigma^{e^{\prime}}(y)$, with $\sigma^{e-e^{\prime}} \sigma^{e^{\prime}}(y)=\sigma^{e}(y)$, contradicting the definition of $S_{\infty}^{-}$. So $e$ is unique.

A symmetric argument shows that for any $s_{i} \in S_{\infty}^{-}$there exists a unique integer $d_{i}$, necessarily with $d_{i} \geqslant 2$, such that $\sigma^{d_{i}}\left(s_{i}\right) \in S_{\infty}^{+}$. Define $t_{i}=\sigma^{d_{i}}\left(s_{i}\right)$; applying the first part of the proof to $t_{i}$, we obtain $e_{i}$ such that $z=\sigma^{e_{i}+d_{i}}\left(s_{i}\right) \in S_{\infty}^{-}$. The definition of $S_{\infty}^{-}$ensures that $e_{i}+d_{i} \geqslant 0$. If $e_{i}+d_{i}>0$, then Remark 5.9(iii) implies that $\sigma^{-e_{i}-d_{i}}$ is defined at $z$ and, of course, $\sigma^{-e_{i}-d_{i}}(z)=s_{i}$, again contradicting the definition of $S_{\infty}^{-}$. Thus $e_{i}=-d_{i}$ and $z=s_{i}$. Finally, beginning with any $t \in S_{\infty}^{+}$, there exists $e \leqslant-2$ with $\sigma^{e}(t)=s_{i}$ for some $i$. Since $\sigma^{d_{i}}\left(s_{i}\right)=t_{i}$ a similar argument forces $e=-d_{i}$ and $t=t_{i}$. This defines the bijection between $S_{\infty}^{+}$and $S_{\infty}^{-}$and it is immediate that $\sigma^{d_{i}}$ is a local isomorphism at $s_{i}$ since the inverse map $\sigma^{-d_{i}}$ is defined at $t_{i}$.

Notation 6.6. This notation will only apply when $A$ is a birationally 2-geometric cg domain for which $\sigma$ is not an automorphism of $Y$; equivalently, by Remark 6.5, when $S_{\infty}^{-} \neq \emptyset$. In this case we fix the notation from Proposition 6.4 and write

$$
\Delta=\max \left\{d_{i}: 1 \leqslant i \leqslant N\right\} .
$$

For $1 \leqslant i \leqslant N$ and $j \in \mathbb{Z}$, define

$$
C_{i j}=\left\{\text { closed points } z \in Y \text { such that } \sigma^{-j} \text { is defined at } z \text { with } \sigma^{-j}(z)=s_{i}\right\}
$$

and write

$$
D=\bigcup_{\substack{-\Delta<j<-\Delta+d_{i} \\ 1 \leqslant i \leqslant N}} C_{i j} \text { and } E=\bigcup_{\substack{0<j<d_{i} \\ 1 \leqslant i \leqslant N}} C_{i j} .
$$

When $C_{i j}$ happens to be a singleton we write $C_{i j}=\left\{c_{i j}\right\}$.

EXAMPLE 6.7. As an illustration of these concepts, consider Example 4.3 for stable $n$. Recall that, in that example, the $S^{ \pm}$are singletons with $S_{\infty}^{-}=S^{-}=\left\{r_{n+1}\right\}$ and $S_{\infty}^{+}=S^{+}=$ $\left\{r_{0}\right\}$; thus $N=1$ with $s_{1}=c_{10}=r_{n+1}$ and $t_{1}=c_{1, n+1}=r_{0}$. The sets $C_{11}, \ldots, C_{1 n}$ are the exceptional divisors $C_{j}^{(n)}$ from (4.3), except that the ordering is reversed: $C_{11}=C_{n}^{(n)}, \ldots, C_{1 n}=$ $C_{1}^{(n)}$. All the other $C_{u v}$ are singletons. Finally $\Delta=d_{1}=n+1$, and $\sigma^{\Delta}$ is defined except at the points $\left\{c_{1,-n}, c_{1,-n+1} \ldots c_{1,-1}\right\}=\left\{r_{2 n+1}, \ldots, r_{n+2}\right\}$.

The following basic properties of the sets $C_{i j}$ will prove useful.

Lemma 6.8. Keep the assumptions and notation from Notation 6.6.

(i) If $y \notin C_{i j}$ for all $i, j$, then $\sigma^{m}$ is defined and a local isomorphism at $y$ for all $m \in \mathbb{Z}$.

(ii) If $y \in C_{i j}$ for some $i$ and $j$, then $\sigma^{d}$ is defined at $y$ for all $d \leqslant-j$ and $d \geqslant-j+d_{i}$.

(iii) If $j \notin\left[1, d_{i}-1\right]$, then $C_{i j}=\left\{c_{i j}\right\}$ is a singleton, with $c_{i j}=\sigma^{j}\left(s_{i}\right)$ a nonsingular point. The map $\sigma$ is defined and a local isomorphism at $c_{i j}$ for $j<0$ and $j \geqslant d_{i}$.

(iv) The sets $C_{i j}$ are pairwise disjoint closed subsets of $Y$.

(v) Suppose that $y \in C_{i j}$ for some $i$ and $j$. If $\sigma^{d}$ is defined at $y$ for some $d \in \mathbb{Z}$, then $\sigma^{d}(y) \in C_{i, j+d}$.

Proof. (i) Suppose that $y \in Y$ is a point where some $\sigma^{m}$ is either not defined or is defined but fails to be a local isomorphism. In the former case, set $z=y$; in the latter case, set $z=\sigma^{m}(y)$ 
and note that $\sigma^{-m}$ is not defined at $z$. In either case, some nonzero power of $\sigma$ is undefined at $z$, so by Lemma 5.5(ii), Lemma 5.7 and Remark 5.9(i,ii), we have either $\sigma^{a}(z) \in S_{\infty}^{+}$or $\sigma^{a}(z) \in S_{\infty}^{-}$ for some $a \in \mathbb{Z}$. Applying Proposition 6.4, if necessary, we conclude that $\sigma^{b}(z)=\sigma^{c}(y) \in S_{\infty}^{-}$ for some $b, c \in \mathbb{Z}$. Thus $y \in C_{i j}$ for some $i, j$.

(ii) When $d \leqslant-j$, Remark 5.9(iii) implies that $\sigma^{d}(y)=\sigma^{d+j} \sigma^{-j}(y)=\sigma^{d+j}\left(s_{i}\right)$ is defined. On the other hand, Proposition 6.4 implies that $\sigma^{d_{i}}\left(s_{i}\right)=t_{i}$ and so, for $d \geqslant-j+d_{i}$, Proposition 5.9(i) implies that $\sigma^{d}(y)=\sigma^{d+j}\left(s_{i}\right)=\sigma^{d-\left(d_{i}-j\right)}\left(t_{i}\right)$ is defined.

(iii) If $j \notin\left[1, d_{i}-1\right]$, then part (ii) implies that $\sigma^{j}\left(s_{i}\right)$ is defined. Hence, for any $z \in C_{i j}$ one has $z=\sigma^{j} \sigma^{-j}(z)=\sigma^{j}\left(s_{i}\right)$. Thus $C_{i j}=\left\{\sigma^{j}\left(s_{i}\right)\right\}$ is a singleton. For $j \geqslant d_{i}$, Proposition 5.9 implies that $c_{i j}$ is nonsingular and $\sigma$ is a local isomorphism at $c_{i j}$. Similarly, when $j<0$, Remark 5.9(iii) says that $c_{i j}$ is nonsingular and that $\sigma^{-1}$ is a local isomorphism at $c_{i, j+1}$. Thus $\sigma$ is a local isomorphism at $c_{i j}$.

(iv,v) Suppose that $y \in C_{i j} \cap C_{i^{\prime} j^{\prime}}$, where $j \geqslant j^{\prime}$. Then $\sigma^{-j}(y)=s_{i}$ and $\sigma^{-j^{\prime}}(y)=s_{i^{\prime}}$. Since $s_{i} \in S_{\infty}^{-}$, Remark 5.9(iii) implies that $\sigma^{j^{\prime}-j}\left(s_{i^{\prime}}\right)$ is defined and thus equal to $s_{i}$. By the definition of $S_{\infty}^{-}$this forces $j=j^{\prime}$ and $s_{i}=s_{i^{\prime}}$, whence $i=i^{\prime}$.

Before completing the proof of (iv), we prove (v). Suppose that $\sigma^{d}$ is defined at $y \in C_{i j}$ but that $\sigma^{d}(y) \notin C_{u v}$ for any $u, v$. Then part (i) implies that $\sigma^{-d-j} \sigma^{d}(y)=\sigma^{-j}(y)$ is defined. But this element must equal $s_{i}$ and so $\sigma^{d}(y) \in C_{i, j+d}$. This contradiction implies that $\sigma^{d}(y) \in C_{u v}$ to begin with. But this implies that $\sigma^{-v+d}(y)=s_{u}$ which, by the previous paragraph, forces $u=i$ and $v=d+j$.

We return to the proof of (iv) and suppose that $y$ is in the closure of some $C_{i j}$. By parts (i) and (ii), and for any $m \gg 0, \sigma^{m}$ is defined in a neighbourhood of $y$. However, if $z \in C_{i j}$, then parts (ii), (iii) and (v) imply that $\sigma^{m}(z)=c_{i, j+m}$ for such an integer $m$. Therefore, by continuity, $\sigma^{m}(y)=c_{i, j+m}$. But then $\sigma^{-j}(y)=\sigma^{-j-m}\left(c_{i, j+m}\right)=s_{i}$ and so $y \in C_{i j}$ after all. Thus $C_{i j}$ is closed.

The significance of the computations in this section is that we tightly prescribe the closed points where $\sigma^{\Delta}$ is either undefined or not a local isomorphism.

Corollary 6.9. Keep the assumptions and notation from Notation 6.6.

(i) The points where $\sigma^{\Delta}$ is undefined are all contained in the finite set $D$.

(ii) The set of points in $U=Y \backslash D$ where $\sigma^{\Delta}$ is not a local isomorphism is contained in the closed (but possibly infinite) set $E$.

Proof. (i) Suppose that $\sigma^{\Delta}$ is undefined at the point $y$. By Lemma 6.8(i), $y \in C_{i j}$ for some $i, j$ and then Lemma 6.8(ii) implies that $-\Delta<j<-\Delta+d_{i}$. Thus $y \in D$. Given $i, j$ with $-\Delta<j<-\Delta+d_{i}$, then $j \leqslant 0$ and so the set $C_{i j}$ is a singleton by Lemma 6.8(iii). As there are finitely many such pairs $\{i, j\}$, the set $D$ is finite.

(ii) If $y \in Y \backslash D$ is a point where $\sigma^{\Delta}$ is not a local isomorphism, then Lemma 6.8(i) again shows that $y \in C_{i j}$ for some $i, j$. Then $\sigma^{-\Delta}$ cannot be defined at $z=\sigma^{\Delta}(y)$. By Lemma 6.8(ii) this forces $0<j<d_{i}$ and $y \in E$.

\section{Constructing the automorphism}

We continue to assume that $A$ is a birationally 2-geometric cg domain, generated in degree one, with graded quotient ring $Q=K\left[t, t^{-1} ; \sigma\right]$ and we maintain the notation from Proposition 6.4 and Notation 6.6. We are now ready to show that the stable scheme $Y$ can be contracted to a second projective scheme $\mathbb{X}$ such that the birational map $\sigma$ descends to an automorphism on $\mathbb{X}$. The idea behind the result is rather easy: if $y \notin \bigcup_{i, j} C_{i j}$ then Lemma 6.8(i) implies that $\sigma^{m}$ 
is defined at $y$ for all $m \in \mathbb{Z}$. On the other hand, Lemma 6.8(ii,iii) implies that $\sigma^{m}$ is defined on all of $C_{i j}$ and $\sigma^{m}\left(C_{i j}\right)=\left\{c_{i, j+m}\right\}$ is a singleton provided that $m \notin\left[-j,-j+d_{i}\right]$. Thus, by patching $U$ and $\sigma^{m}\left(U^{\prime}\right)$ for appropriate open subsets $U$ and $U^{\prime}$ of $Y$ and some $m \gg 0$, we may hope to construct a new scheme $Y^{\prime}$ for which the images of the $C_{i j}$ are singletons $\left\{c_{i j}^{\prime}\right\}$. At this point it is easy to define an action of $\sigma$ on $Y^{\prime}$ by setting $\sigma\left(c_{i j}^{\prime}\right)=c_{i, j+1}^{\prime}$. That this idea works forms the content of the next theorem.

TheOrem 7.1. Let $A$ be a birationally 2-geometric cg domain that is generated in degree one and write $Y=Y_{n}$ for some stable value of $n$ with its induced birational action of $\sigma$. Then there exists a surjective birational morphism $\pi: Y \rightarrow \mathbb{X}$, where $\mathbb{X}=\mathbb{X}_{n}$ is a projective surface, such that:

(i) $\tilde{\sigma}=\pi \sigma \pi^{-1}$ is an automorphism of $\mathbb{X}$, and

(ii) $\pi^{-1}: \mathbb{X} \rightarrow Y$ is defined except at a finite set $P$ of nonsingular closed points of $\mathbb{X}$, each of which lies on a dense $\widetilde{\sigma}$-orbit.

REMARK 7.2. In the special case of Example $4.3, \mathbb{X} \cong \mathbb{P}^{2}$ and the morphism $\pi: Y \rightarrow \mathbb{X}$ is simply the map $\gamma_{n}$ from that example that blows up $\mathbb{P}^{2}$ at the $n$ points $\left\{r_{1}, \ldots, r_{n}\right\}$.

Proof. If $S_{\infty}^{-}=\emptyset$ then $\sigma$ is an automorphism by Remark 6.5 and so we can simply take $\mathbb{X}=$ $Y$ and let $\pi$ be the identity. We may therefore assume that $S_{\infty}^{-} \neq \emptyset$ and hence, by Remark 6.5 again, that $S_{\infty}^{+} \neq \emptyset$.

Define the integer $\Delta$ and the sets $C_{i j}, D$ and $E$ as in Notation 6.6. Set $U_{1}=Y \backslash D$ and notice that, by construction, $E \subset U_{1}$. By Corollary 6.9(i,ii) we know that $\sigma^{\Delta}$ is defined at every point of $U_{1}$, while $\sigma^{\Delta}$ is defined and a local isomorphism on the open subset $U_{1} \backslash E$ of $Y$. By the definition of the $C_{i j}$, we also know that $U_{2}=\sigma^{\Delta}\left(U_{1}\right)$ is equal to $Y \backslash E$, and so, in particular, $U_{2}$ is open in $Y$ with $Y=U_{1} \cup U_{2}$. By Lemma 6.8(ii), $\sigma^{\Delta}(E)=\bigcup\left\{c_{i j}: 1 \leqslant i \leqslant N, \Delta<j<\right.$ $\left.\Delta+d_{i}\right\}$ is a finite set of points.

We now want to construct the surface $\mathbb{X}$. Formally, we will do this by glueing two open sets, but the construction is probably clearer if we first indicate what is happening at the level of elements. So, set $H=Y \backslash\left\{C_{i j}: 1 \leqslant i \leqslant N, j \in \mathbb{Z}\right\}$, let $\widetilde{H}$ be an isomorphic copy of $H$ and for each $i, j$ as above define an abstract point $\widetilde{c}_{i j}$. Then the new scheme $\mathbb{X}$ will simply be $\widetilde{H} \cup\left\{\widetilde{c}_{i j}: 1 \leqslant i \leqslant N, j \in \mathbb{Z}\right\}$, with the projection $\pi: Y \rightarrow \mathbb{X}$ defined by the given isomorphism $H \rightarrow \widetilde{H}$ together with $\pi\left(C_{i j}\right)=\widetilde{c}_{i j}$.

To make this formal, and especially to define the topology on $\mathbb{X}$, recall that $Y$ is covered by the open sets $U_{1}$ and $U_{2}$. Let $V_{1}=U_{2}$ and $V_{2}=U_{2}$ be two new copies of $U_{2}$, and set $V_{12}=U_{2} \backslash \sigma^{\Delta}(E)$ and $V_{21}=U_{1} \backslash E$; thus $V_{12}$ is an open subset of $V_{1}$ and $V_{21}$ is open in $V_{2}$. The morphism $\left.\sigma^{\Delta}\right|_{U_{1}}$ restricts to an isomorphism $f: V_{21} \rightarrow V_{12}$. Now think of $V_{1}$ and $V_{2}$ as new quasi-projective schemes and forget their embeddings in $Y$. Then we can use the isomorphism $f$ to glue $V_{2}$ and $V_{1}$ along their open subschemes $V_{21}$ and $V_{12}$ to obtain a new scheme $\mathbb{X}$. Define a morphism $\pi: Y \rightarrow \mathbb{X}$ by defining $\left.\pi\right|_{U_{1}}=\sigma^{\Delta}: U_{1} \rightarrow U_{2}=V_{1}$ and letting $\left.\pi\right|_{U_{2}}: U_{2} \rightarrow U_{2}=V_{2}$ be the identity map. It is immediate that these definitions agree on $U_{1} \cap U_{2}=V_{21}$, and so $\pi$ is indeed a well-defined morphism. It is obviously surjective and birational. By construction, $\pi$ is a local isomorphism except possibly at points in the set $E=Y \backslash U_{2}$, and so the morphism $\pi^{-1}$ is defined except possibly at the finite set of points $P=\pi(E) \subset \mathbb{X}$.

As was noted earlier, if $C_{i j} \subseteq E$, then Lemma 6.8(iii,v) implies that $\widetilde{c}_{i j}=\pi\left(C_{i j}\right) \cong \sigma^{\Delta}\left(C_{i j}\right)$ is indeed a closed point of $\mathbb{X}$, while for $C_{i j} \nsubseteq \subseteq E$ it follows from Lemma 6.8(iii,iv) that both $C_{i j}$ and $\widetilde{c}_{i j}=\pi\left(C_{i j}\right)$ consist of a single closed point. Since $H=Y \backslash\left\{C_{i j}: 1 \leqslant i \leqslant N, j \in \mathbb{Z}\right\} \subset U_{1} \cap U_{2}$, this justifies the comments of the paragraph before last. We next want prove that the birational $\operatorname{map} \widetilde{\sigma}=\pi \sigma \pi^{-1}: \mathbb{X} \rightarrow \mathbb{X}$ is actually an automorphism and once again this is particularly easy 
to describe at the level of sets: $\widetilde{\sigma}\left(\widetilde{c}_{i j}\right)=\widetilde{c}_{i, j+1}$, while the action of $\widetilde{\sigma}$ on $\widetilde{H}=\pi(H)$ is induced from the fact that $\sigma$ is defined on $H$.

Formally, let $E^{\prime}=E \cup\left\{s_{i}: 1 \leqslant i \leqslant N\right\}$ and $U_{2}^{\prime}=Y \backslash E^{\prime} \subseteq U_{2}$. Also, write $P^{\prime}=\pi\left(Z^{\prime}\right)=$ $P \cup \pi\left(\left\{s_{i}\right\}\right)$ and $V_{2}^{\prime}=\pi\left(U_{2}^{\prime}\right)=\mathbb{X} \backslash P^{\prime}$. By Lemma 6.8(i,iii), $\sigma: U_{2}^{\prime} \rightarrow U_{2}$ is defined and therefore an open immersion. Since $\left.\pi\right|_{U_{2}}: U_{2} \rightarrow V_{2}$ is an isomorphism, this implies that $\widetilde{\sigma}=\pi \sigma \pi^{-1}$ : $V_{2}^{\prime} \rightarrow V_{2}$ is also an immersion. Next, set $U_{1}^{\prime}=U_{1} \backslash \bigcup_{i} C_{i,-\Delta}$ and $V_{1}^{\prime}=\pi\left(U_{1}^{\prime}\right) \subseteq V_{1}$. Recall that $V_{1}$ was defined to be a copy of $U_{2}$ and write this identification as $g: V_{1} \rightarrow U_{2}$. By the definition of $\left.\pi\right|_{U_{1}}$, we see that $g \pi=\sigma^{\Delta}$ as morphisms from $U_{1}$ to $U_{2}$. Since $g$ maps $V_{1}^{\prime}$ isomorphically onto $U_{2}^{\prime}$, it follows that $\widetilde{\sigma}^{\prime}=g^{-1} \sigma g$ is defined as a morphism from $V_{1}^{\prime}$ to $V_{1}$ and is even an immersion. As birational maps, $g^{-1} \sigma g=\pi \sigma^{-\Delta} \sigma \sigma^{\Delta} \pi^{-1}=\pi \sigma \pi^{-1}$. Thus $\tilde{\sigma}$ and $\tilde{\sigma}^{\prime}$ represent the same birational map $\mathbb{X} \rightarrow \mathbb{X}$, and so they patch together to give a morphism $\widetilde{\sigma}: V_{1}^{\prime} \cup V_{2}^{\prime} \rightarrow V_{1} \cup V_{2}$.

From the definitions of $D$ and $E$ in Notation 6.6 it is clear that $Y=U_{1}^{\prime} \cup U_{2}^{\prime}$. Applying $\pi$, this gives $V_{i}^{\prime} \cup V_{2}^{\prime}=\mathbb{X}$. Thus $\widetilde{\sigma}: \mathbb{X} \rightarrow \mathbb{X}$ is a globally defined birational morphism which, by the previous paragraph, is a local isomorphism at every point. Thus the inverse birational map is defined at every point of $\mathbb{X}$ and so $\widetilde{\sigma}$ must be an isomorphism. It is also clear that, at the level of points, $\widetilde{\sigma}$ has the form described in the paragraph before last; in particular $\widetilde{\sigma}\left(\widetilde{c}_{i j}\right)=\widetilde{c}_{i, j+1}$ for each $i, j$.

It remains to prove that $\mathbb{X}$ is projective, which follows from the next, more general proposition.

Lemma 7.3. Let $W$ be an integral scheme of finite type. Let $\mathcal{M}_{1}, \mathcal{M}_{2}$ be invertible sheaves on $W$ with $M_{i} \subseteq \mathrm{H}^{0}\left(X, \mathcal{M}_{i}\right)$ such that each $M_{i}$ generates $\mathcal{M}_{i}$. Suppose that the map $W \rightarrow$ $\mathbb{P}\left(M_{1}^{*}\right)$ is an immersion. If $M$ denotes the image of $M_{1} \otimes M_{2}$ in $\mathrm{H}^{0}\left(X, \mathcal{M}_{1} \otimes \mathcal{M}_{2}\right)$, then the map $W \rightarrow \mathbb{P}\left(M^{*}\right)$ is an immersion.

Proposition 7.4. Let $Y$ be a projective $k$-scheme and $\mathbb{X}$ be an integral $k$-scheme of finite type with a birational surjective morphism $\pi: Y \rightarrow \mathbb{X}$. Assume that $\mathbb{X}$ has an automorphism $\tau$ and a finite set $P$ of nonsingular closed points such that:

(i) $\pi^{-1}$ is defined on $U=\mathbb{X} \backslash P$, and

(ii) every point of $P$ lies on an infinite orbit of $\tau$.

Then $\mathbb{X}$ is a projective scheme.

Proof. We begin by proving that $\mathbb{X}$ is separated and proper, for which we use the valuative criteria from [14, Section II.4]. By [14, Exercise II.4.11(c)], we need only consider discrete valuation rings in the criteria. So, fix a discrete valuation ring $R$ with field of fractions $L$ and write $\iota: B=\operatorname{Spec} L \hookrightarrow C=\operatorname{Spec} R$ for the induced morphism. Let $c$ denote the closed point of $C$ and write $b$ for the generic point of $C$ (and $B$ ). We also have the structure map $f: \mathbb{X} \rightarrow$ Spec $k$ and assume that there exist morphisms $\alpha: C \rightarrow \operatorname{Spec} k, \beta: B \rightarrow \mathbb{X}$ such that $\alpha \iota=f \beta$.

We first prove that $\mathbb{X}$ is separated, so assume that there exist two morphisms $\gamma_{i}: C \rightarrow \mathbb{X}$ both of which make (7.5)(I) commute.

(I)

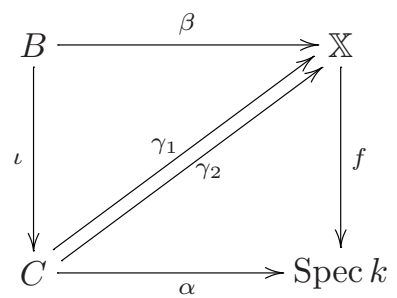

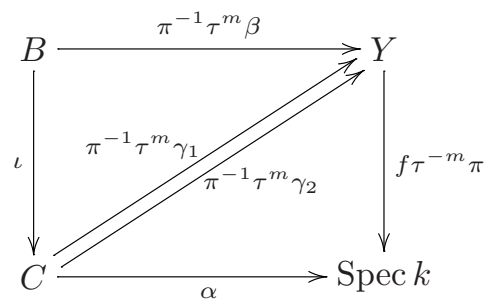


Write $y_{1}=\gamma_{1}(c)$ and $y_{2}=\gamma_{2}(c)$. By assumption (ii) we choose $m \gg 0$ such that $\tau^{m}\left(y_{1}\right)$ and $\tau^{m}\left(y_{2}\right)$ lie in $U=\mathbb{X} \backslash P$; this forces $\tau^{m} \beta(b) \in U$ as well. Then $\pi^{-1} \tau^{m} \gamma_{i}: C \rightarrow Y$ is a well-defined morphism for $i=1,2$, and similarly $\pi^{-1} \tau^{m} \beta: B \rightarrow Y$ is well-defined. Moreover, the diagram (7.5)(II) commutes. Since $Y$ is separated, [14, Theorem II.4.3] implies that $\pi^{-1} \tau^{n} \gamma_{1}=\pi^{-1} \tau^{n} \gamma_{2}$ and hence $\gamma_{1}=\gamma_{2}$. By [14, Theorem II.4.3], again, this implies that $\mathbb{X}$ is separated.

We now prove properness. By hypothesis, there exists $m \gg 0$ such that $\mathbb{X}$ is covered by the open sets $U \cong \pi^{-1}(U)$ and $\tau^{m}(U)$, so $\mathbb{X}$ is noetherian. By the Valuative Criterion for Properness [14, Theorem II.4.7] it therefore suffices to find a morphism $\gamma: C \rightarrow \mathbb{X}$ making (7.6)(I) commute.

(I)

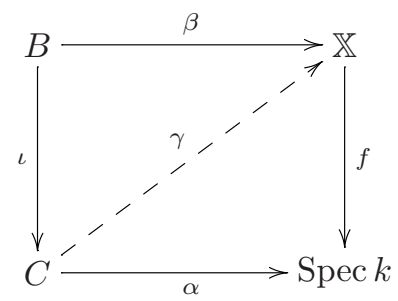

(II)

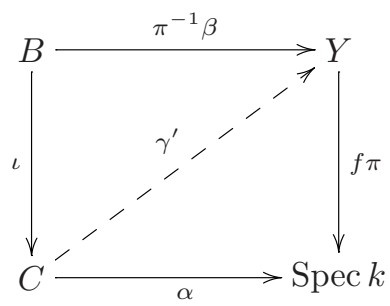

First, if $x=\beta(b)$ is a closed point of $\mathbb{X}$ then clearly putting $\gamma(c)=\gamma(b)=y$ gives a well-defined map $\gamma$ making (7.6)(I) commute. Alternatively, if $x$ is not a closed point, then $x \notin P$ and so $\pi^{-1}$ is defined at $x$. As $Y$ is proper, there then exists $\gamma^{\prime}: C \rightarrow Y$ making the diagram (7.6)(II) commute. Thus $\gamma=\pi \gamma^{\prime}$ makes (7.6)(I) commute and $\mathbb{X}$ is proper.

Before proving that $\mathbb{X}$ is projective, we prove the following easy facts. The constant sheaf of rational functions on $Y$ and on $\mathbb{X}$ will be written $\mathcal{K}$.

Sublemma 7.7. Keep the notation from the Proposition 7.6. Suppose that $\mathcal{M} \subset \mathcal{K}$ is an invertible sheaf on $Y$ and set $\mathcal{F}=\pi_{*} \mathcal{M} \subset \mathcal{K}$. Then:

(i) the reflexive hull $\mathcal{L}=\mathcal{F}^{* *}=\mathcal{H}^{\circ} m_{\mathcal{O}_{\mathbb{X}}}\left(\mathcal{H}_{o} m_{\mathcal{O}_{\mathbb{X}}}\left(\mathcal{F}, \mathcal{O}_{\mathbb{X}}\right), \mathcal{O}_{\mathbb{X}}\right)$ of $\mathcal{F}$ is invertible;

(ii) $\mathcal{L}$ is the unique reflexive (or invertible) sheaf on $\mathbb{X}$ satisfying $\left.\mathcal{L}\right|_{U}=\left.\mathcal{F}\right|_{U}$;

(iii) $\mathcal{L}^{\otimes n}$ is the reflexive hull of $\mathcal{F}^{n}$, where the multiplication takes place inside $\mathcal{K}$.

Proof. (i) Since $\left.\mathcal{F}\right|_{U}=\left.\mathcal{M}\right|_{\pi^{-1}(U)}$ under the identification of $\mathcal{O}_{U}$ with $\mathcal{O}_{\pi^{-1}(U)}$, the sheaf $\mathcal{L}$ is locally free on $U$. On the other hand, if $p \in P$, then $\mathcal{O}_{\mathbb{X}, p}$ is regular and hence factorial by [11, Theorem 19.19] and so any reflexive ideal of $\mathcal{O}_{\mathbb{X}, p}$ is cyclic. Thus $\mathcal{L}$ is locally free at each point of $\mathbb{X}$.

(ii) We need only prove this locally at a closed point $y \in P$. If $\mathcal{Q}$ is a second reflexive sheaf with $\left.\mathcal{Q}\right|_{U}=\left.\mathcal{F}\right|_{U}$, then certainly $\left(\mathcal{Q}_{y}\right)_{\mathfrak{p}}=\left(\mathcal{F}_{y}\right)_{\mathfrak{p}}=\left(\mathcal{L}_{y}\right)_{\mathfrak{p}}$ for all height one prime ideals $\mathfrak{p}$ of the regular local ring $\mathcal{O}_{\mathbb{X}, y}$. But $\mathcal{Q}_{y}=\bigcap\left\{\left(\mathcal{Q}_{y}\right)_{\mathfrak{p}}: \mathfrak{p}\right.$ height 1$\}$. Thus $\mathcal{Q}_{y}=\mathcal{L}_{y}$ and hence $\mathcal{Q}=\mathcal{L}$.

(iii) Since $\left.\mathcal{L}^{\otimes n}\right|_{U}=\left.\mathcal{F}^{n}\right|_{U}=\left.\left(\pi_{*}\left(\mathcal{M}^{\otimes n}\right)\right)\right|_{U}$, this follows from part (ii) applied to $\mathcal{M}^{\otimes n}$.

We return to the proof of the proposition and prove that $\mathbb{X}$ is projective. Let $\mathcal{M} \subset \mathcal{K}$ be any very ample invertible sheaf on $Y$ and set $\mathcal{F}=\pi_{*} \mathcal{M}$ with reflexive hull $\mathcal{L}=\mathcal{F}^{* *}$. By the sublemma $\mathcal{L}$ is locally free. Since $\mathcal{M}$ is generated by its sections $M=\mathrm{H}^{0}(Y, \mathcal{M})$ and $M=\mathrm{H}^{0}(\mathbb{X}, \mathcal{F}) \subseteq \mathrm{H}^{0}(\mathbb{X}, \mathcal{L})$, the sections $\mathrm{H}^{0}(\mathbb{X}, \mathcal{L})$ do at least generate the sheaf $\left.\mathcal{L}\right|_{U}=\left.\mathcal{F}\right|_{U} \cong$ $\left.\mathcal{M}\right|_{\pi^{-1}(U)}$ as an $\mathcal{O}_{U}$-module. Since $P=\mathbb{X} \backslash U$ is finite and $\mathbb{X}$ is proper, it therefore follows from [13, Corollary 1.14] that, for any $n \gg 0$, the sheaf $\mathcal{L}^{\otimes n}$ is generated by its global sections $V_{1}=\mathrm{H}^{0}\left(\mathbb{X}, \mathcal{L}^{\otimes n}\right)$. For $r \in \mathbb{Z}$, write $\mathcal{N}_{r}=\left(\tau^{-r}\right)^{*}\left(\mathcal{L}^{\otimes n}\right)$.

Let $\theta: \mathbb{X} \rightarrow \mathbb{P}\left(V_{1}^{*}\right)=\mathbb{P}^{d}$ denote the map determined by $V_{1}$ and write $V_{2}=\mathrm{H}^{0}\left(Y, \mathcal{M}^{\otimes n}\right)$. Then Sublemma 7.7(iii) implies that $V_{2}=\mathrm{H}^{0}\left(\mathbb{X}, \pi_{*} \mathcal{M}^{\otimes n}\right) \subseteq \mathrm{H}^{0}\left(Y, \mathcal{L}^{\otimes n}\right)=V_{1}$. Since $\mathcal{M}^{\otimes n}$ is very 
ample, $V_{2}$ defines a closed immersion of $Y$ into $\mathbb{P}\left(V_{2}^{*}\right)$ and hence an immersion $\theta^{\prime}: \pi^{-1}(U) \hookrightarrow$ $\mathbb{P}\left(V_{2}^{*}\right)$. Since $\theta^{\prime}$ factors through $\theta$, this ensures that $\theta$ defines an immersion of $U$ into $\mathbb{P}\left(V_{1}^{*}\right)$.

By hypothesis (ii) of the proposition, $\mathbb{X}=U \cup \tau^{m}(U)$ for $m \gg 0$ and, for some such $m$, we consider the map $\alpha: \mathbb{X} \rightarrow \mathbb{P}\left(V_{3}^{*}\right)$ determined by $V_{3}=\mathrm{H}^{0}\left(\mathbb{X}, \mathcal{N}_{0} \otimes \mathcal{N}_{m}\right)$. Since the $\mathcal{N}_{r}$ are generated by their sections, we can apply Lemma 7.3 with $W=U$ to show that the restriction of $\alpha$ to $U$ is an immersion. For exactly the same reasons the map $\theta_{1}: \mathbb{X} \rightarrow \mathbb{P}^{d}$ determined by $\mathrm{H}^{0}\left(\mathbb{X}, \mathcal{N}_{m}\right)$ immerses $\tau^{m}(U)$ into $\mathbb{P}^{d}$ and so the restriction of $\alpha$ to $\tau^{m}(U)$ is also an immersion.

Since $\alpha$ is defined on all of $\mathbb{X}=U \cup \tau^{m}(U)$, we can define $C$ to be the closure in $\mathbb{P}\left(V_{3}^{*}\right)$ of $\alpha(\mathbb{X})$. Since $\mathbb{X}$ is proper and irreducible, $C=\alpha(\mathbb{X})$ and obviously the corresponding map $\beta: \mathbb{X} \rightarrow C$ is birational. The conclusion of the previous paragraph implies that $\beta$ is a local isomorphism at every point of $\mathbb{X}$. Therefore, for any point $c \in C$ we can define an inverse map to $\beta$ in a neighbourhood of $c$ and so the inverse birational map $\beta^{-1}$ is defined at every point of $C$. Hence $\beta: \mathbb{X} \rightarrow C$ is an isomorphism. In other words, $\alpha: \mathbb{X} \rightarrow \mathbb{P}\left(V_{3}^{*}\right)$ is a closed immersion and $\mathbb{X}$ is projective.

REMARK 7.8. For future reference we note that the last part of the proof of Proposition 7.4 proves the following fact: Suppose that $\mathcal{M}$ is a very ample invertible sheaf on $Y$ and let $\mathcal{B}=\left(\pi_{*} \mathcal{M}\right)^{* *}$. Then, for all $q \gg 0$, the sheaf $\mathcal{B} \otimes\left(\tau^{q}\right)^{*} \mathcal{B}$ is an ample invertible sheaf on $\mathbb{X}$.

Although the construction of the scheme $\mathbb{X}_{n}$ in Theorem 7.1 depends upon $n$, this is unimportant as there is a natural isomorphism $\mathbb{X}_{n} \cong \mathbb{X}_{n+1}$ for any stable value of $n$. Before proving this we need a technical lemma.

Lemma 7.9. Let $y \in Y=Y_{n}$, for a fixed stable value of $n$. Then there exists $m_{0} \geqslant 0$ such that, for all $m \geqslant m_{0}$ and $r \geqslant 0, \sigma^{m}$ is defined at $y$ and the birational map $\phi^{-r}$ is defined at $\sigma^{m}(y)$.

Proof. Let $w \in Y$ and suppose that $\phi^{-r}$ is not defined at $w$ for some $r \geqslant 1$. If we choose the minimal such $r$ then $\phi^{-1}$ is undefined at $\widetilde{w}=\phi^{-r+1}(w) \in Y_{n+r-1}$. Since $n+r-1$ is again a stable value, Remark 5.9(i) implies that $\sigma^{e}(\widetilde{w}) \in S_{n+r-1}^{-}$for some $e \leqslant 0$. Thus $\sigma^{e+r-1}=$ $\psi^{r-1} \sigma^{e} \phi^{-r+1}$ is defined at $w$ and, by Lemma 4.4(i), $\sigma^{e+r-1}(w) \in S^{-}$. Using Notation 6.6 and Remark 5.9(ii), it follows that $w \in C_{i, j}$ for some $i, j$.

Return to the proof of the lemma and suppose that $y \notin C_{i j}$ for any $i, j$. Then Lemma 6.8(i) implies that $y_{m}=\sigma^{m}(y)$ is defined for all $m$. If $\phi^{-r}$ is undefined at $w=y_{m}$ for some $m \geqslant 0$ and $r \geqslant 0$, then the previous paragraph shows that $y_{m} \in C_{i j}$ for some $i, j$. This forces $y \in C_{i, j-m}$, a contradiction. Thus in this case we may take $m_{0}=0$.

Otherwise, $y \in C_{i j}$ some $i, j$, and so $\sigma^{e}(y)=t_{i} \in S_{\infty}^{+}$for $e=d_{i}-j$. Set $z=t_{i}$ and notice that, by Lemma 4.4(ii), $\phi^{-r}$ is defined at $z$ for all $r \geqslant 0$. For $d \geqslant 0$, the map $\sigma^{d}$ is defined and a local isomorphism at $z$ by Proposition 5.9. Hence $\phi^{-r}=\psi^{d} \phi^{-r-d} \sigma^{-d}$ is also defined at $z_{d}=\sigma^{d}(z)$ for all $r \geqslant 0$. Finally, $\sigma^{e+d}(y)=z_{d}$ for all $d \geqslant 0$ and so the conclusion of the lemma holds with $m_{0}=\max (0, e)$.

Lemma 7.10. The scheme $\mathbb{X}_{n}$ is independent of the stable value of $n$ in the sense that, for any such $n$, there exists an isomorphism $\alpha_{n}: \mathbb{X}_{n+1} \rightarrow \mathbb{X}_{n}$ such that $\pi_{n} \phi_{n}=\alpha_{n} \pi_{n+1}$.

Proof. Let $y \in \mathbb{X}_{n}$. By Theorem 7.1(ii), $\pi_{n}^{-1}$ is defined at $\widetilde{\sigma}_{n}^{e}(y)$ for all $e \gg 0$, say for all $e \geqslant e_{0}$. Set $z=\widetilde{\sigma}_{n}^{e_{0}}(y)$. Then by Lemma 7.9 , we may choose $e_{1} \geqslant 0$ such that $\phi_{n}^{-1}$ is defined 
at $\widetilde{z}_{e}=\sigma_{n}^{e} \pi_{n}^{-1}(z)=\pi_{n}^{-1} \widetilde{\sigma}_{n}^{e}(z)$, for all $e \geqslant e_{1}$. So, for all $w$ in some neighbourhood of $y$ we may define

$$
\alpha_{n, m}^{-1}(w)=\widetilde{\sigma}_{n+1}^{-m} \circ \pi_{n+1} \circ \phi_{n}^{-1} \circ \pi_{n}^{-1} \circ \widetilde{\sigma}_{n}^{m}(w) \text { for } m \geqslant m_{0}=e_{0}+e_{1} .
$$

By Lemma 5.3, we have the equality $\sigma_{n+1} \phi_{n}^{-1}=\phi_{n}^{-1} \sigma_{n}$ of birational maps. Also, $\widetilde{\sigma}_{n} \pi_{n}=\pi_{n} \sigma_{n}$ as birational maps by the definition of $\widetilde{\sigma}_{n}$. Thus $\alpha_{n, m}^{-1}=\widetilde{\sigma}_{n+1}^{-m} \pi_{n+1} \phi_{n}^{-1} \pi_{n}^{-1} \widetilde{\sigma}_{n}^{m}=\pi_{n+1} \phi_{n}^{-1} \pi_{n}^{-1}$ as birational maps, independent of the choice of $m \geqslant m_{0}$. Thus the birational map $\alpha_{n}^{-1}=$ $\pi_{n+1} \phi_{n}^{-1} \pi_{n}^{-1}$ is defined in a neighbourhood of $y$ which, since $y$ was arbitrary, implies that $\alpha_{n}^{-1}$ is globally defined. A similar (but easier) argument shows that the birational map $\alpha_{n}=\pi_{n} \phi_{n} \pi_{n+1}^{-1}$ is globally defined, and so $\alpha_{n}$ is an isomorphism.

We end the section by giving a more algebraic interpretation of the scheme $\mathbb{X}$; the set of closed points in $\mathbb{X}$ is canonically isomorphic to a set $\widetilde{P}_{\mathrm{qgr}}$ of $k$-point modules in qgr- $A$. This is both suggestive and curious. It is curious since, as is shown by [16, Theorem 0.1], $\widetilde{P}_{\text {qgr }}$ is definitely not represented by a scheme of finite type when $A$ is a naïve blowup algebra. However, it does suggest that there should be some category of modules over $A$ which is represented by $\mathbb{X}$.

Formally, for $m \geqslant n$, a closed point $y \in Y_{m}$ can be identified with a truncated $k$-point module $M_{y}$ of length $m+1$. Mimicking the definition in Section 2 , let $\widetilde{\mathcal{P}}_{m}$ denote the set of isomorphism classes of all such truncated point modules and let $\widetilde{\mathcal{P}}=\lim _{\leftarrow} \widetilde{\mathcal{P}}_{m}$ denote the set of isomorphism classes of point modules corresponding these truncated modules. Finally, let $\widetilde{\mathcal{P}}_{\text {qgr }}$ denote the image of $\widetilde{\mathcal{P}}$ in qgr- $A$. Although we will not prove it here, the set $\widetilde{\mathcal{P}}_{\text {qgr }}$ is the image in qgr of the set of all point modules from gr- $A$ and hence also the set of all point modules in qgr- $A$ in the sense of the introduction.

Corollary 7.11. Pick a stable value of $n$ and set $\mathbb{X}=\mathbb{X}_{n}$. There is a bijective correspondence $\rho$ from the set of closed points in the scheme $\mathbb{X}$ to the set of objects in $\widetilde{\mathcal{P}}_{\text {qgr }}$ with the following property: given $\widetilde{y} \in \mathbb{X}$, write $\widetilde{y}=\pi(y)$ for some $y \in Y$, say corresponding to the truncated point module $M=M_{y} \in \widetilde{P}_{n}$. Then $\rho(\widetilde{y})$ is the image $\bar{N}$ in qgr- $A$ of any point module $N \in \widetilde{\mathcal{P}}$ satisfying $M \cong N_{\leqslant n}$.

Proof. Let $y \in Y=Y_{n}$, for a fixed stable value of $n$ and recall the definitions from Notation 6.6 and Theorem 7.1. We first claim that the map $\pi^{\prime}: \widetilde{P}_{n} \rightarrow \widetilde{\mathcal{P}}_{\text {qgr }}$ given by $y \mapsto \bar{N}$ is well-defined.

To prove this, pick $m \geqslant 0$ by Lemma 7.9 so that the map $\phi^{-r}$ is defined at $\sigma^{m}(y)$ for all $r \geqslant 0$. We now translate this into module theory. Let $M_{y} \in \widetilde{\mathcal{P}}_{n}$ be the truncated point module corresponding to the point $y \in Y_{n}$. By Corollary 3.5(ii), the maps $\phi_{d}$ are surjective for $d \geqslant n$, and so there is a sequence of points $y_{d} \in Y_{d}$ with $y_{n}=y$ and $\phi\left(y_{d+1}\right)=y_{d}$ for all $d \geqslant 0$. Corresponding to this sequence is a point module $N$ such that $N_{\leqslant n} \cong M_{y}$. The truncation shift $L=N[m]_{\geqslant 0}$ is also a truncated point module but now, by (2.1) and (2.2), $L_{\leqslant n}$ corresponds to the point $\sigma^{m}(y) \in Y$. Since $\phi^{-r}$ is defined at $\sigma^{m}(y)$ for all $r \geqslant 0, L$ must be the unique point module $L^{\prime}$ with $L_{\leqslant n}^{\prime} \cong L_{\leqslant n}$. In other words, $N[m]_{\geqslant 0}$ and hence $N_{\geqslant m}$ are uniquely determined by $M_{y}$ and $y$. Since $N$ and $N_{\geqslant m}$ have the same image in $\widetilde{\mathcal{P}}_{\text {qgr }}$, this says that $\pi^{\prime}$ is well-defined.

In order to show that the correspondence $\rho$ is well-defined and bijective, it suffices to prove the following: given closed points $x_{1} \neq x_{2} \in Y$ then $\pi\left(x_{1}\right)=\pi\left(x_{2}\right)$ if and only if $\pi^{\prime}\left(x_{1}\right)=\pi^{\prime}\left(x_{2}\right)$. By the construction of $\pi$ in Theorem 7.1, $\pi\left(x_{1}\right)=\pi\left(x_{2}\right)$ if and only if $x_{1}, x_{2} \in C_{i j}$ for some $i, j$. But in this case, Lemma 6.8(ii,iii,v) implies that $\sigma^{m}\left(x_{1}\right)=\sigma^{m}\left(x_{2}\right)=c_{i, j+m}$ for $m \gg 0$. Now suppose that $N(i)$ are point modules with $N(i)_{\leqslant n}=M_{x_{i}}$ for $i=1,2$. Then, just as in the previous paragraph, it follows from $(2.1)$ and $(2.2)$ that $L(i)=N(i)[m]_{\geqslant 0}$ is a truncated point module corresponding to $\sigma^{m}\left(x_{i}\right)$, which is independent of $i$. In other words, $N(1)$ and $N(2)$ 
have isomorphic tails, $\overline{N(1)} \cong \overline{N(2)}$, and $\rho$ is well-defined. Conversely, if $\pi^{\prime}\left(x_{1}\right)=\pi^{\prime}\left(x_{2}\right)$, then it follows from the argument of the previous paragraph that $\sigma^{m}\left(x_{1}\right)=\sigma^{m}\left(x_{2}\right)$ for all $m \gg 0$. Thus $x_{1}=x_{2} \in C_{i j}$ for some $i, j$ and so $\pi\left(x_{1}\right)=\pi\left(x_{2}\right)$.

\section{Naïve blowing up}

We now have the data required for the statement of the Main Theorem 1.1 and in the next three sections we show how to use this to describe the relevant cg domains as naïve blowup algebras. In this section we give the definitions and some of the basic properties of these algebras. Despite their name, naïve blowups have properties that are very different from the classical case, just look at Corollary 1.3. They have been studied in detail in $[\mathbf{1 6}]$; but only for the case where one is blowing up a single closed point, whereas for applications in this paper we need to be able to naïvely blowup a zero-dimensional subscheme of the given scheme and this complicates several of the results from [16]. A detailed examination of these more general rings is given in the companion paper $[\mathbf{2 3}]$, to which the reader is referred for the details behind the assertions given here.

The following assumptions will be in force throughout the section.

Assumptions 8.1. Fix an integral projective scheme $X$ of dimension $d \geqslant 2$ with an automorphism $\tau$ and field of rational functions $K=k(X)$. The corresponding sheaf of rational functions will be written $\mathcal{K}$. Let $\mathcal{L} \subset \mathcal{K}$ be an invertible sheaf and let $\mathcal{I}$ be an ideal sheaf such that the associated scheme $Z_{\mathcal{I}}=\mathcal{V}(\mathcal{I})$ is zero-dimensional. We allow $\mathcal{I}=\mathcal{O}_{X}$ but, to avoid trivialities, we assume that if $s \in Z_{\mathcal{I}}$ then $s$ lies on an infinite $\tau$-orbit in $X$.

Given an $\mathcal{O}_{X}$-module $\mathcal{M}$, write $\tau^{*}(\mathcal{M})=\mathcal{M}^{\tau}$. If $\mathcal{M} \subseteq \mathcal{K}$, set $\mathcal{M}_{0}=\mathcal{O}_{X}$ and $\mathcal{M}_{n}=\mathcal{M} \mathcal{M}^{\tau}$. $\ldots \cdot \mathcal{M}^{\tau^{n-1}}$ for $n>0$, under the multiplication induced from $\mathcal{K}$. Of course, $\mathcal{M}_{n} \cong \mathcal{M} \otimes \mathcal{M}^{\tau} \otimes$ $\ldots \otimes \mathcal{M}^{\tau^{n-1}}$ when $\mathcal{M}$ is invertible but we will also need use the construction for $\mathcal{M}=\mathcal{L} \mathcal{I}$ where $\mathcal{M M}^{\tau}$ is typically not isomorphic to $\mathcal{M} \otimes \mathcal{M}^{\tau}$.

Given this data, and regarding $\mathcal{K}\left[t, t^{-1} ; \tau\right]$ as a sheaf of $\mathcal{O}_{X}$-algebras in the natural way, we can form the bimodule algebra

$$
\mathcal{R}\left(X, Z_{\mathcal{I}}, \mathcal{L}, \tau\right)=\bigoplus_{n \geqslant 0} \mathcal{R}_{n} t^{n} \subseteq \mathcal{K}\left[t, t^{-1} ; \tau\right]
$$

where $\mathcal{R}_{n}=\mathcal{L}_{n} \mathcal{I}_{n} \cong \mathcal{L}_{n} \otimes \mathcal{I}_{n}$ for $n \geqslant 0$. The definition of $\mathcal{R}=\mathcal{R}\left(X, Z_{\mathcal{I}}, \mathcal{L}, \tau\right)$ in [16] or $[\mathbf{2 3}]$ is slightly different, although equivalent, since it is given in terms of $\mathcal{O}_{X}$-bimodules. One advantage of the present definition is that the multiplication in $\mathcal{R}$ is induced from that in $\mathcal{K}\left[t, t^{-1} ; \tau\right]$ and is given by the natural map

$$
\mathcal{R}_{m} t^{m} \otimes \mathcal{R}_{n} t^{n} \longrightarrow \mathcal{R}_{m} t^{m} \mathcal{R}_{n} t^{n}=\mathcal{R}_{m} \mathcal{R}_{n}^{\tau^{m}} t^{m+n}=\mathcal{R}_{m+n} t^{m+n} .
$$

We remark that, as is noted in $\left[6\right.$, p. 252], $\mathcal{R}\left(X, Z_{\mathcal{I}}, \mathcal{L}, \tau\right)$ is not a sheaf of algebras in the usual sense, since the presence of $\sigma$ means one has to play a game of musical chairs with the open sets. The naïve blowup algebra of $X$ at $Z_{\mathcal{I}}$ is then defined to be the ring of global sections

$$
R\left(X, Z_{\mathcal{I}}, \mathcal{L}, \tau\right)=\mathrm{H}^{0}(X, \mathcal{R})=\bigoplus_{n \geqslant 0} R_{n}=\bigoplus_{n \geqslant 0} \bar{R}_{n} t^{n},
$$

where $\bar{R}_{n}=\mathrm{H}^{0}\left(X, \mathcal{I}_{n} \otimes \mathcal{L}_{n}\right)$ for $n \geqslant 0$.

As a matter of convention we write a graded subspace of $R$ or $Q(R)$ as $I=\bigoplus I_{n}=\bigoplus \bar{I}_{n} t^{n}$, for some $\bar{I}_{n} \subset k(X)$, but we do not use the overline for subsets of $\mathcal{R}$.

Although we have implicitly assumed that $\mathcal{I} \neq \mathcal{O}_{X}$ in these definitions, they make perfect sense when $\mathcal{I}=\mathcal{O}_{X}$. In that case one obtains the bimodule algebra $\mathcal{B}(X, \mathcal{L}, \sigma)$ 
$=\mathcal{R}(X, \emptyset, \mathcal{L}, \tau)$ whose ring of global sections is the familiar twisted homogeneous coordinate ring $B(X, \mathcal{L}, \tau)=R(X, \emptyset, \mathcal{L}, \tau)=\bigoplus_{n \geqslant 0} \mathrm{H}^{0}\left(X, \mathcal{L}_{n}\right) t^{n}$ from [6]. We note in passing that $R\left(X, Z_{\mathcal{I}}, \mathcal{L}, \tau\right) \subseteq B(X, \mathcal{L}, \tau)$ for any $Z_{\mathcal{I}}$.

By definition, a graded right $\mathcal{R}$-module is a quasi-coherent $\mathcal{O}_{X}$-module $\mathcal{M}=\bigoplus_{i \in \mathbb{Z}} \mathcal{M}_{n}$ together with a right $\mathcal{O}_{X}$-module map $\mu: \mathcal{M} \otimes \mathcal{R} \rightarrow \mathcal{M}$ satisfying the usual axioms. The module $\mathcal{M}$ is called coherent (as an $\mathcal{R}$-module) if each $\mathcal{M}_{n}$ is a coherent $\mathcal{O}_{X}$-module, with $\mathcal{M}_{n}=0$ for $n \ll 0$, such that the natural map $\mu_{n}: \mathcal{M}_{n} \otimes \mathcal{R}_{1}^{\tau^{n}} \rightarrow \mathcal{M}_{n+1}$ is surjective for $n \gg 0$. (By [23, Lemma 2.11], this is equivalent to the somewhat more general definition from [28]. The superscript of $\tau^{n}$ that appears here does not appear in that lemma since the two papers use slightly different notation; in $[\mathbf{2 3}] \mathcal{R}_{1}$ is a bimodule into which one absorbs the action of $\tau$.) The bimodule algebra $\mathcal{R}$ is (right) noetherian if all (right) ideals of $\mathcal{R}$ are coherent.

The usual definitions for graded modules over cg algebras also apply to bimodule algebras. In particular, the graded right $\mathcal{R}$-modules form an abelian category $\mathrm{Gr}-\mathcal{R}$, with homomorphisms graded of degree zero. The subcategory of coherent modules is denoted gr- $\mathcal{R}$ and is abelian when $\mathcal{R}$ is noetherian [16, Proposition 2.10]. Let tors- $\mathcal{R}$ denote by the full subcategory of gr- $\mathcal{R}$ consisting of coherent $\mathcal{O}_{X}$-modules, and write qgr- $\mathcal{R}$ for the quotient category gr- $\mathcal{R} /$ tors- $\mathcal{R}$. The category qgr- $\mathcal{R}$ is called the naïve blowup of $X$ at $Z_{\mathcal{I}}$ (see, also, Theorem 8.5).

Naïve blowup algebras only behave well if the sequence $\left\{\mathcal{R}_{n}=\mathcal{L}_{n} \mathcal{I}_{n}\right\}$ is ample in the following sense. Assume that $\mathcal{M} \subset \mathcal{K}$. Then the sequence $\left\{\mathcal{M}_{n}: n \geqslant 0\right\}$ is ample, equivalently $\mathcal{M}$ is $\tau$-ample, if for every coherent $\mathcal{O}_{X}$-module $\mathcal{F}$ the sheaf $\mathcal{F} \otimes_{\mathcal{O}_{X}} \mathcal{M}_{n}$ is generated by its global sections and $\mathrm{H}^{i}\left(X, \mathcal{F} \otimes \mathcal{M}_{n}\right)=0$ for all $i>0$ and all $n \gg 0$. If $\mathcal{M}$ is an invertible sheaf, then $\tau$-ampleness is a subtle but well-understood concept (see [15]), but much less is known when $\mathcal{M}$ is not invertible.

A basic method for relating $\mathcal{R}$ to $R$ is given by the following result; see [16, Theorem 2.12] or, for a more general version, [28, Theorem 5.2]:

Theorem 8.5 (Van den Bergh). Keep Assumptions 8.1 and let $\mathcal{R}=\mathcal{R}\left(X, Z_{\mathcal{I}}, \mathcal{L}, \tau\right)$. Assume that $\mathcal{R}$ is noetherian and that $\left\{\mathcal{R}_{n}: n \in \mathbb{N}\right\}$ is an ample sequence of $\mathcal{O}_{X}$-modules. Then the naïve blowup algebra $R=R\left(X, Z_{\mathcal{I}}, \mathcal{L}, \tau\right)$ is noetherian, and there is an equivalence of categories qgr- $\mathcal{R} \rightarrow$ qgr- $R$ given by $\mathrm{H}^{0}(X,-)$.

In order to use this theorem to pass from $R$ to $\mathcal{R}$ we need to know when $\mathcal{R}$ is noetherian and when $\left\{\mathcal{R}_{n}\right\}$ is an ample sequence. The answer is provided by the next two results.

Theorem 8.6. Keep Assumptions 8.1 and assume that $\mathcal{L}$ is $\tau$-ample and that each point $z \in Z_{\mathcal{I}}$ lies on a Zariski dense $\tau$-orbit. Then the sequences $\left\{\mathcal{R}_{n}=\mathcal{L}_{n} \otimes \mathcal{I}_{n}\right\}$ and $\left\{\mathcal{R}_{n}^{\tau^{-n}}\right\}$ are ample.

Proof. The ampleness of $\left\{\mathcal{R}_{n}\right\}$ is proved in [23, Theorem 3.1]. In order to prove the second claim write $\mathcal{R}_{n}^{\tau^{-n}}=\mathcal{S} \otimes \mathcal{S}^{\alpha} \otimes \ldots \otimes \mathcal{S}^{\alpha^{n-1}}$ for $\mathcal{S}=\mathcal{L}^{\tau^{-1}} \otimes \mathcal{I}^{\tau^{-1}}$ and $\alpha=\tau^{-1}$. By $[\mathbf{1 5}$, Corollary 5.1], $\mathcal{L}^{\alpha}$ is $\alpha$-ample and clearly the other hypotheses of the theorem also hold for $\alpha$ and $\mathcal{S}$. So $\left[\mathbf{2 3}\right.$, Theorem 3.1] also shows that $\mathcal{S}$ is $\alpha$-ample; equivalently, that $\left\{\mathcal{R}_{n}^{\tau^{-n}}\right\}$ is ample.

The noetherian property for $\mathcal{R}$ and $R$ requires stronger hypotheses. For $z \in X$, the $\tau$-orbit $\left\{\tau^{i}(z): i \in \mathbb{Z}\right\}$ is called critically dense if it is infinite and each infinite subset of this orbit is (Zariski) dense in $X$. We say that $Z_{\mathcal{I}}$ is saturating if either $Z_{\mathcal{I}}=\emptyset$ or, for each point $z \in Z_{\mathcal{I}}$, the $\tau$-orbit $\left\{\tau^{i}(z): i \in \mathbb{Z}\right\}$ is critically dense. 
Proposition 8.7. Keep Assumptions 8.1. Then the following hold.

(i) The bimodule algebra $\mathcal{R}=\mathcal{R}\left(X, Z_{\mathcal{I}}, \mathcal{L}, \tau\right)$ is noetherian if and only if $Z_{\mathcal{I}}$ is a saturating subset of $X$.

(ii) Assume that $\mathcal{L}$ is $\tau$-ample and that either $Z_{\mathcal{I}}=\emptyset$ or that each point $z \in Z_{\mathcal{I}}$ lies on a Zariski dense $\tau$-orbit. Then $R\left(X, Z_{\mathcal{I}}, \mathcal{L}, \tau\right)$ is noetherian if and only if $Z_{\mathcal{I}}$ is saturating.

Proof. (i) See [23, Proposition 2.12].

(ii) If $Z_{\mathcal{I}}$ is saturating then this follows from part (i) combined with Theorems 8.6 and 8.5. If $Z_{\mathcal{I}}$ is not saturating then it follows from [23, Proposition 3.16].

\section{Surjectivity in large degree}

The aim of this section is to show that if a cg algebra is 'close to' being a naïve blowup algebra, then it actually equals one (see Theorem 9.2 for the precise statement). This is a generalization of [3, Theorem 4.1], which proved the analogous result for twisted homogeneous coordinate rings, and the present proof closely follows the argument from [3].

The following assumptions and notation will remain in force through to the end of the proof of Theorem 9.2.

Assumptions 9.1. Fix an integral projective scheme $X$ of dimension at least 2 with sheaf of rational functions $\mathcal{K}$, an automorphism $\tau$ and a $\tau$-ample invertible sheaf $\mathcal{L} \subset \mathcal{K}$. Let $\mathcal{I} \subseteq \mathcal{O}_{X}$ be an ideal sheaf defining a zero-dimensional subscheme $Z_{\mathcal{I}}$ of $X$ and write $\mathcal{R}_{n}=\mathcal{L}_{n} \mathcal{I}_{n} \cong \mathcal{L}_{n} \otimes \mathcal{I}_{n}$ for each $n \geqslant 0$. Let $P_{1}$ be a finite set of closed points of $X$, with $\operatorname{Supp} Z_{\mathcal{I}} \subseteq P_{1}$, and assume that each $x \in P_{1}$ lies on a (Zariski) dense $\tau$-orbit of $X$.

For $n \geqslant 1$ write $P_{n}=\bigcup_{j=0}^{n-1} \tau^{-j}\left(P_{1}\right)$; thus, $P_{n} \supseteq \bigcup_{j=0}^{n-1} \tau^{-j}\left(\operatorname{Supp} Z_{\mathcal{I}}\right)=\operatorname{Supp} \mathcal{O}_{X} / \mathcal{I}_{n}$. We continue to use the notation from the previous section; in particular a naïve blowup algebra will be written in the form $R=R\left(X, Z_{\mathcal{I}}, \mathcal{L}, \tau\right)=\bigoplus \bar{R}_{n} t^{n} \subseteq K\left[t, t^{-1} ; \tau\right]$, with $\bar{R}_{n}=\mathrm{H}^{0}\left(X, \mathcal{R}_{n}\right)$, as described in (8.4).

We can now state the first form of the surjectivity result. For rings generated in degree one, this will be further refined in Corollary 9.13.

Theorem 9.2. Keep the data from Assumptions 9.1. Let $T=\bigoplus_{n \geqslant 0} \bar{T}_{n} t^{n}$ be a $c g$ noetherian subring of the naïve blowup algebra $R=R\left(X, Z_{\mathcal{I}}, \mathcal{L}, \tau\right)$ such that:

(i) for $n \gg 0, \bar{T}_{n}$ generates the sheaf $\mathcal{R}_{n}$, and

(ii) for $n \gg 0$, the birational map $\theta_{n}: X \rightarrow \mathbb{P}\left(\bar{T}_{n}^{*}\right)$ defined by the sections $\bar{T}_{n} \subseteq \bar{R}_{n} \subseteq$ $\mathrm{H}^{0}\left(X, \mathcal{L}_{n}\right)$ restricts to give an immersion $\left(X \backslash P_{n}\right) \hookrightarrow \mathbb{P}\left(\bar{T}_{n}^{*}\right)$.

Then $T=R$ up to a finite-dimensional vector space and $Z_{\mathcal{I}}$ is saturating.

Note that, by (i), the sections $\bar{T}_{n}$ do generate $\mathcal{L}_{n}\left(X \backslash P_{n}\right)$ and so part (ii) makes sense. The proof of Theorem 9.2 will be obtained through a series of lemmas and so the assumptions and notation from the theorem will remain in force throughout that proof. The first lemma is a standard application of $\tau$-ample sheaves.

Lemma 9.3. Let $V \subseteq \mathrm{H}^{0}(X, \mathcal{F})$ be a vector space that generates the coherent sheaf $\mathcal{F}$ and pick $r \in \mathbb{Z}$. Then: 
(i) for $p \gg 0$, the natural homomorphism $\alpha: V \otimes_{k} H^{0}\left(X, \mathcal{R}_{p}^{\tau^{r}}\right) \rightarrow H^{0}\left(X, \mathcal{F} \otimes \mathcal{R}_{p}^{\tau^{r}}\right)$ is surjective;

(ii) for $p \gg 0$, the natural homomorphism $\beta: V^{\tau^{p}} \otimes_{k} H^{0}\left(X, \mathcal{R}_{p}\right) \rightarrow H^{0}\left(X, \mathcal{F}^{\tau^{p}} \otimes \mathcal{R}_{p}\right)$ is surjective.

Proof. (i) Set $V^{\prime}=V^{\tau^{-r}} \subseteq \mathrm{H}^{0}\left(X, \mathcal{F}^{\tau^{-r}}\right)$. Clearly $\alpha$ is surjective if and only if the map $\alpha^{\prime}: V^{\prime} \otimes \mathrm{H}^{0}\left(X, \mathcal{R}_{p}\right) \rightarrow \mathrm{H}^{0}\left(X, \mathcal{F}^{\tau^{-r}} \otimes \mathcal{R}_{p}\right)$ is surjective. Thus we may assume that $r=0$.

There is a short exact sequence $0 \rightarrow \mathcal{H} \rightarrow V \otimes_{k} \mathcal{O}_{X} \rightarrow \mathcal{F} \rightarrow 0$ for the appropriate sheaf $\mathcal{H}$. Tensoring this sequence with $\mathcal{R}_{p}$ gives an exact sequence

$$
0 \longrightarrow \mathcal{G} \stackrel{\alpha}{\longrightarrow} \mathcal{H} \otimes \mathcal{R}_{p} \longrightarrow V \otimes_{k} \mathcal{R}_{p} \longrightarrow \mathcal{F} \otimes \mathcal{R}_{p} \longrightarrow 0 .
$$

The sheaf $\mathcal{G}$ is supported at the finite set of points $P_{p}$ where $\mathcal{R}_{p}$ need not be locally free, and so $\mathrm{H}^{j}(X, \mathcal{G})=0$ for $j>0$. Therefore, if $\mathcal{A}_{p}=\operatorname{Coker}(\alpha)$, then $\mathrm{H}^{j}\left(X, \mathcal{A}_{p}\right)=\mathrm{H}^{j}\left(X, \mathcal{H} \otimes \mathcal{R}_{p}\right)$ for all $j>0$ and all $p \geqslant 0$. As $\left\{\mathcal{R}_{p}\right\}$ is ample, this implies that $\mathrm{H}^{j}\left(X, \mathcal{A}_{p}\right)=0$, for all $j>0$ and $p \gg 0$. Thus, taking global sections of the last displayed equation gives the required surjection $V \otimes_{k} \mathrm{H}^{0}\left(X, \mathcal{R}_{p}\right) \rightarrow \mathrm{H}^{0}\left(X, \mathcal{F} \otimes \mathcal{R}_{p}\right)$.

(ii) By applying $\tau^{-p}$ to the given equation, it suffices to prove surjectivity of the homomorphism $V \otimes \mathrm{H}^{0}\left(X, \mathcal{R}_{p}^{\tau^{-p}}\right) \rightarrow \mathrm{H}^{0}\left(X, \mathcal{F} \otimes \mathcal{R}_{p}^{\tau^{-p}}\right)$. Now use the proof of part (i), combined with the second assertion from Theorem 8.6.

Lemma 9.4. The natural map $\mathrm{H}^{0}\left(X, \mathcal{R}_{m} \otimes \mathcal{R}_{n}^{\tau^{m}}\right) \rightarrow \mathrm{H}^{0}\left(X, \mathcal{R}_{n+m}\right)$ is a surjection for any $m, n \geqslant 0$.

Proof. Since $\mathcal{R}_{m+n}=\mathcal{R}_{m} \mathcal{R}_{n}^{\tau^{m}}$, the natural surjective map from the tensor product to the product fits into a short exact sequence $0 \rightarrow \mathcal{H} \rightarrow \mathcal{R}_{m} \otimes \mathcal{R}_{n}^{\tau^{m}} \rightarrow \mathcal{R}_{m+n} \rightarrow 0$ for some sheaf $\mathcal{H}$. Since $\mathcal{R}_{m}$ is locally free except at a finite set of points, $\mathcal{H}$ is supported on a finite set of points and thus $\mathrm{H}^{1}(X, \mathcal{H})=0$. Taking global sections of the short exact sequence gives the required surjection.

Lemma 9.5. The ring $R$ is finitely generated as a left and right $T$-module. Moreover, $R$ is noetherian, and $Z_{\mathcal{I}}$ is a saturating subscheme of $X$.

Proof. Fix $n \gg 0$ for which $\bar{T}_{n}$ generates $\mathcal{R}_{n}$. Then Lemma 9.3(i), with $\mathcal{F}=\mathcal{R}_{n}$ and $V=$ $\bar{T}_{n} \subseteq \mathrm{H}^{0}\left(X, \mathcal{R}_{n}\right)$, together with Lemma 9.4, implies that the multiplication map

$$
\bar{T}_{n} \otimes \bar{R}_{p}^{\tau^{n}}=\bar{T}_{n} \otimes \mathrm{H}^{0}\left(X, \mathcal{R}_{p}^{\tau^{n}}\right) \longrightarrow \mathrm{H}^{0}\left(X, \mathcal{R}_{n} \otimes \mathcal{R}_{p}^{\tau^{n}}\right) \longrightarrow \mathrm{H}^{0}\left(X, \mathcal{R}_{n+p}\right)=\bar{R}_{n+p}
$$

is surjective for all $p \gg 0$; say for all $p \geqslant p_{0}$. Thus $R$ is finitely generated by $R_{0} \oplus \ldots \oplus R_{p_{0}+n-1}$ as a left $T$-module. An analogous argument, using Lemma 9.3(ii), shows that $R_{T}$ is finitely generated.

As $T$ is noetherian, this certainly implies that $R$ is noetherian. By Assumption 9.1 the hypotheses of Proposition 8.7(ii) are therefore satisfied and, by that result, $Z_{\mathcal{I}}$ is saturating.

Lemma 9.6. It suffices to prove Theorem 9.2 for some pair of Veronese rings $T^{(d)} \subseteq R^{(d)}$.

Proof. The Veronese $\operatorname{ring} R^{(d)} \cong R\left(X, Z_{\mathcal{I}_{d}}, \mathcal{L}_{d}, \tau^{d}\right)$ is again a naïve blowup algebra with data satisfying Assumptions 9.1 and so the hypotheses of Theorem 9.2 do carry over to the Veronese rings $T^{(d)} \subseteq R^{(d)}$. 
Assume that the theorem holds for $T^{(d)} \subseteq R^{(d)}$; thus $T_{s}^{(d)}=R_{s}^{(d)}$ for all $s \gg 0$, and hence $\bar{T}_{s d}=\bar{R}_{s d}$ for all such $s$. By hypothesis, we can find $n_{0}$ such that $\bar{T}_{n}$ generates $\mathcal{R}_{n}$ for all $n \geqslant n_{0}$. Pick $n$ satisfying $n_{0} \leqslant n \leqslant n_{0}+d$. Then Lemma 9.4(i), with $p=s d, r=n$ and $V=$ $\bar{T}_{n} \subseteq \mathrm{H}^{0}\left(X, \mathcal{R}_{n}\right)$, together with Lemma 9.4, implies that the multiplication map

$$
\bar{T}_{n} \otimes \bar{T}_{s d}^{\tau^{n}}=\bar{T}_{n} \otimes \bar{R}_{s d}^{\tau^{n}} \longrightarrow \bar{R}_{n+s d}
$$

is surjective for $s \gg 0$, say for all $s \geqslant s_{0}$. Since there are only finitely many choices of $n$, we may assume that $s_{0}$ is chosen independently of the choice of $n$. Hence, $\bar{T}_{u}=\bar{R}_{u}$ for all $u \geqslant$ $n_{0}+s_{0} d$.

The key step in the proof of Theorem 9.2 is to relate ideals of $R$ to $\tau$-invariant ideal sheaves on $X$. This is given by the next result.

Proposition 9.7. Let $M=\bigoplus_{n \geqslant 0} \bar{M}_{n} t^{n}$ be a two-sided ideal of the naïve blowup algebra $R$. Then there exists a $\tau$-invariant sheaf of ideals $\mathcal{J} \subseteq \mathcal{O}_{X}$ such that $\mathrm{H}^{0}\left(X, \mathcal{J} \mathcal{R}_{n}\right)=\bar{M}_{n}$ for all $n \gg 0$.

Proof. Let $\mathcal{R}=\mathcal{R}\left(X, Z_{\mathcal{I}}, \mathcal{L}, \tau\right)=\bigoplus_{n \geqslant 0} \mathcal{R}_{n} t^{n}$ be the bimodule algebra associated to $R$, in the sense of (8.2). By Theorem 8.5 the sequence $\left\{\mathcal{R}_{n}\right\}$ is ample and by Proposition 8.7 $\mathcal{R}$ is noetherian, so Theorem 8.5 applies. By that result, there exists $n_{0} \geqslant 0$ and a coherent submodule $\mathcal{G}=\bigoplus_{n \geqslant 0} \mathcal{G}_{n} t^{n} \subseteq \mathcal{R}$ such that $\bar{M}_{n}=\mathrm{H}^{0}\left(X, \mathcal{G}_{n}\right)$ for each $n \geqslant n_{0}$. By the definition of coherent $\mathcal{R}$-modules in Section 8 and increasing $n_{0}$ if necessary, we may assume that $\mathcal{G}_{n+1}=$ $\mathcal{G}_{n} \mathcal{R}_{1}^{\tau^{n}}$ for all $n \geqslant n_{0}$. Since $\mathcal{R}_{m}=\mathcal{R}_{1} \cdot \ldots \cdot \mathcal{R}_{1}^{\tau^{m-1}}$, induction implies that $\mathcal{G}_{n+m}=\mathcal{G}_{n} \mathcal{R}_{m}^{\tau^{n}}$ for $m>0$ and $n \geqslant n_{0}$. Equivalently, if $\mathcal{H}_{n}=\mathcal{G}_{n} \mathcal{L}_{n}^{-1} \cong \mathcal{G}_{n} \otimes \mathcal{L}_{n}^{-1}$, then $\mathcal{H}_{n} \subseteq \mathcal{O}_{X}$ is a sheaf of ideals satisfying

$$
\mathcal{H}_{n+m}=\mathcal{H}_{n} \mathcal{I}_{m}^{\tau^{n}} \subseteq \mathcal{H}_{n} \subseteq \mathcal{H}_{n_{0}} \quad \text { for each } n \geqslant n_{0} \text { and } m \geqslant 0 .
$$

As $\mathcal{G}_{m+n_{0}}=\mathcal{G}_{n_{0}} \mathcal{R}_{m}^{\tau^{n_{0}}}$ for all $m \geqslant 0$, the ampleness of $\left\{\mathcal{R}_{m}\right\}$ implies that $\mathcal{G}_{n}$ is generated by its sections $\bar{M}_{n}$ for all $n \gg 0$.

Now consider the fact that $M$ is also a left ideal of $R$. The hypotheses on $R$ and $\mathcal{R}$ are leftright symmetric and so the equivalence of categories, Theorem 8.5, also applies to left ideals. Thus there also exists a coherent left submodule $\mathcal{G}^{\prime}=\bigoplus_{m \geqslant 0} \mathcal{G}_{m}^{\prime} t^{m} \subseteq \mathcal{R}$ such that $\mathrm{H}^{0}\left(Y, \mathcal{G}_{n}^{\prime}\right)=$ $\bar{M}_{n}$ for all $n \gg 0$. By ampleness, again, $\mathcal{G}_{n}^{\prime}$ is generated by its sections $\bar{M}_{n}$ for all $n \gg 0$. Thus $\mathcal{G}_{n}^{\prime}=\mathcal{G}_{n}$ for all $n \gg 0$, which we may assume happens for all $n \geqslant n_{0}$. As $\mathcal{G}^{\prime}$ is a left submodule of $\mathcal{R}$ this implies that $\mathcal{R}_{m} \mathcal{G}_{n}^{\tau^{m}}=\mathcal{R}_{m}\left(\mathcal{G}_{n}^{\prime}\right)^{\tau^{m}} \subseteq \mathcal{G}_{n+m}^{\prime}=\mathcal{G}_{n+m}$ for all $m \geqslant 0$ and $n \geqslant n_{0}$. Equivalently

$$
\mathcal{I}_{m} \mathcal{H}_{n}^{\tau^{m}} \subseteq \mathcal{H}_{n+m} \text { for each } n \geqslant n_{0} \text { and } m \geqslant 0 .
$$

Set $P_{\infty}=\left\{\tau^{i}\left(P_{1}\right): i \in \mathbb{Z}\right\}$; thus $P_{\infty} \supseteq \operatorname{Supp} \mathcal{O}_{X} / \mathcal{I}_{r}$ for all $r \geqslant 0$. If $\mathcal{N} \subseteq \mathcal{O}_{X}$ is a sheaf of ideals, write $\mathcal{S}(\mathcal{N})$ for the largest sheaf of ideals with the property that $\operatorname{Supp} \mathcal{S}(\mathcal{N}) / \mathcal{N} \subseteq P_{\infty}$. Clearly $\mathcal{S}(\mathcal{I N})=\mathcal{S}(\mathcal{N})$ and so it follows from (9.8) and (9.9) that

$$
\mathcal{S}\left(\mathcal{H}_{n_{0}}\right)^{\tau}=\mathcal{S}\left(\mathcal{H}_{n_{0}}^{\tau}\right)=\mathcal{S}\left(\mathcal{I} \mathcal{H}_{n_{0}}^{\tau}\right) \subseteq \mathcal{S}\left(H_{n_{0}+1}\right) \subseteq \mathcal{S}\left(\mathcal{H}_{n_{0}}\right)
$$

Since $X$ is noetherian, this forces $\mathcal{S}\left(\mathcal{H}_{n_{0}}\right)^{\tau}=\mathcal{S}\left(\mathcal{H}_{n_{0}}\right)$. Thus $\mathcal{J}=\mathcal{S}\left(\mathcal{H}_{n_{0}}\right)$ is a $\tau$-invariant sheaf of ideals and we will show that it satisfies the conclusions of the proposition.

We therefore need to show that $\mathcal{G}_{n}=\mathcal{J R}_{n}$ in high degree. Tensoring this statement by $\mathcal{L}_{n}^{-1}$, we need to show that $\mathcal{H}_{n}=\mathcal{J I}_{n}$ for $n \gg 0$. Since $X$ is noetherian, there exists an ideal sheaf $\mathcal{A}$ such that $\mathcal{H}_{n_{0}} \supseteq \mathcal{J} \mathcal{I}_{n_{0}} \mathcal{A}$, and such that $\mathcal{O}_{X} / \mathcal{A}$ is supported at a finite number of points of 
$P_{\infty}$. For any $m \geqslant 0,(9.9)$ gives

$$
\mathcal{H}_{n_{0}+m} \supseteq \mathcal{I}_{m} \mathcal{H}_{n_{0}}^{\tau^{m}} \supseteq \mathcal{I}_{m} \mathcal{I}_{n_{0}}^{\tau^{m}} \mathcal{A}^{\tau^{m}} \mathcal{J}^{\tau^{m}}=\mathcal{A}^{\tau^{m}} \mathcal{I}_{n_{0}+m} \mathcal{J}
$$

On the other hand, (9.8) implies that

$$
\mathcal{H}_{n_{0}+m}=\mathcal{H}_{n_{0}} \mathcal{I}_{m}^{\tau^{n_{0}}} \supseteq \mathcal{I}_{m}^{\tau^{n_{0}}} \mathcal{I}_{n_{0}} \mathcal{A} \mathcal{J}=\mathcal{A} \mathcal{I}_{n_{0}+m} \mathcal{J}
$$

Since $\mathcal{O}_{X} / \mathcal{A}$ is supported at a finite number of points, each lying on an infinite $\tau$-orbit, $\mathcal{A}^{\tau^{m}}+$ $\mathcal{A}=\mathcal{O}_{X}$ for $m \gg 0$. Combining the last two displayed equations therefore shows that $\mathcal{H}_{n_{0}+m} \supseteq$ $\mathcal{J I}_{n_{0}+m}$, for all $m \gg 0$.

As $\mathcal{J}$ is $\tau$-invariant, $\operatorname{Supp} \mathcal{O}_{X} / \mathcal{J}$ is a proper $\tau$-invariant closed subspace of $X$. Since each $x \in P_{\infty}$ lies on a dense $\tau$-orbit, this forces $W=\operatorname{Supp}\left(\mathcal{O}_{X} / \mathcal{J}\right) \cap \operatorname{Supp}\left(\mathcal{O}_{X} / \mathcal{I}_{n}\right)$ to be empty. But $\mathcal{J} \cap \mathcal{I}_{n} / \mathcal{J I}_{n}$ is supported on $W$. Thus $\mathcal{J} \cap \mathcal{I}_{n}=\mathcal{J I}_{n}$ for all $n$ and so the previous paragraph implies that $\mathcal{H}_{n} \supseteq \mathcal{J} \cap \mathcal{I}_{n}$ for all $n \gg n_{0}$. On the other hand, $\mathcal{H}_{n} \subseteq \mathcal{H}_{n_{0}} \subseteq \mathcal{S}\left(\mathcal{H}_{n_{0}}\right)=\mathcal{J}$ by (9.8) and $\mathcal{H}_{n} \subseteq \mathcal{I}_{n}$ simply because $\mathcal{G}_{n} \subseteq \mathcal{R}_{n}=\mathcal{L}_{n} \mathcal{I}_{n}$. Thus $\mathcal{H}_{n} \subseteq \mathcal{J} \cap \mathcal{I}_{n}=\mathcal{J I}_{n}$ and hence $\mathcal{H}_{n}=\mathcal{J I}_{n}$ for all $n \gg n_{0}$. Equivalently, $\mathcal{G}_{n}=\mathcal{J R}_{n}$ and $\bar{M}_{n}=\mathrm{H}^{0}\left(X, \mathcal{J} \mathcal{R}_{n}\right)$, for all $n \gg 0$.

REMARK 9.10. The proof of Proposition 9.7 also proves the following result: let $\mathcal{G}=\bigoplus \mathcal{G}_{n} t^{n}$ be an ideal of the bimodule algebra $\mathcal{R}$. Then there exists a $\tau$-invariant sheaf of ideals $\mathcal{J} \subseteq \mathcal{O}_{X}$ such that $\mathcal{G}_{n}=\mathcal{J R}_{n}$ for all $n \gg 0$.

Proof of Theorem 9.2. First, by Lemma 9.6 we may replace $R$ and $T$ by Veronese rings and thereby assume that conditions (i) and (ii) of the theorem actually hold for all $n \geqslant 1$. By Assumptions 9.1, $R$ and hence $T$ are contained in $k(X)\left[t, t^{-1} ; \tau\right]$ and by condition (i), $T_{1} \neq 0 ;$ say $0 \neq t_{1}=a t \in T_{1}$ for some $a \in k(X)$. Therefore, $k(X)\left[t, t^{-1} ; \tau\right]=k(X)\left[t_{1}, t_{1}^{-1} ; \tau_{1}\right]$ and one even has $\tau=\tau_{1}$ since they differ by conjugation by the element $a \in k(X)$. Since $T$ is noetherian, it has a graded Goldie quotient ring which, by universality, will have the form $Q(T)=F\left[t_{1}, t_{1}^{-1} ; \tau\right] \subseteq Q(R) \subseteq k(X)\left[t_{1}, t_{1}^{-1} ; \tau\right]$ for some field $F \subseteq k(X)$ invariant under $\tau$.

We claim that $F=k(X)$. To see this, set $V=X \backslash P_{1}$ and note that, by conditions (i) and (ii), $\bar{T}_{1}$ generates $\left.\mathcal{L}\right|_{V}$ and induces an immersion $\chi: V \hookrightarrow \mathbb{P}\left(\bar{T}_{1}^{*}\right)$. Fix a basis $\left\{s_{j}: 0 \leqslant j \leqslant r\right\}$ of $\bar{T}_{1}$, write $V_{0}=\left\{x \in V:\left(s_{0}\right)_{x} \notin \mathfrak{m}_{x} \mathcal{L}_{x}\right\}$ and let $U_{0}=\left\{x_{0} \neq 0\right\}$ be the standard open affine subset of $\mathbb{P}\left(\bar{T}_{1}^{*}\right)$ with $\mathcal{O}\left(U_{0}\right)=k\left[x_{1} x_{0}^{-1}, \ldots, x_{r} x_{0}^{-1}\right]$. Then $\chi\left(V_{0}\right)$ is open in its closure inside $U_{0}$. As in the proof of [14, Theorem II.7.1], the morphism $\left.\chi\right|_{V_{0}}$ is then given by the map of rings $\alpha: k\left[x_{1} x_{0}^{-1}, \ldots, x_{r} x_{0}^{-1}\right] \rightarrow \Gamma\left(V_{0}, \mathcal{O}_{V_{0}}\right)$ defined by $x_{j} x_{0}^{-1} \mapsto s_{j} s_{0}^{-1}$, and it follows that $\Gamma\left(V_{0}, \mathcal{O}_{V_{0}}\right)$ is a localization of the image of $\alpha$. On the other hand, as $X$ is an integral scheme, it has the same field of rational functions as both $V$ and $V_{0}$ and so $k(X)$ is generated as a field by the $s_{j} s_{0}^{-1} \in F$. Thus $k(X)=F$.

It follows from the previous paragraph that $Q(T)=Q(R)=k(X)\left[t_{1}, t_{1}^{-1} ; \tau\right]$ and so $R / T$ is Goldie torsion as a right (or left) $T$-module. By Lemma 9.5, $R / T$ is therefore a finitely generated, torsion right $T$-module and so the left annihilator $J_{\ell}=\ell-\operatorname{ann}_{T}(R / T)$ is a nonzero ideal of $T$. Similarly, $J_{r}=r-\operatorname{ann}_{T}(R / T)$ and $M=J_{r} J_{\ell}$ are nonzero ideals of $T$. But $M$ is also an ideal of $R$. So, if $M=\bigoplus M_{n}$ then Proposition 9.7 produces a nonzero $\tau$-invariant ideal sheaf $\mathcal{J} \subseteq \mathcal{O}_{X}$ such that

$$
\mathrm{H}^{0}\left(X, \mathcal{J} \mathcal{R}_{n}\right) t^{n}=M_{n} \quad \text { for all } n \gg 0 .
$$

We next consider the factor bimodule algebra $\mathcal{R} / \mathcal{J R}=\bigoplus_{n \geqslant 0} \mathcal{R}_{n} t^{n} / \mathcal{J} \mathcal{R} t^{n}$. Let $W \subseteq X$ be the $\tau$-invariant subscheme defined by $\mathcal{J}$. As every point of Supp $Z_{\mathcal{I}_{n}} \subseteq P_{n}$ lies on a dense $\tau$-orbit, and $W$ is a proper $\tau$-invariant subscheme of $X$, it follows that $Z_{\mathcal{I}_{n}} \cap W=\emptyset$ and hence that $\mathcal{R}_{n} / \mathcal{J R}_{n}=\mathcal{R}_{n} \otimes_{\mathcal{O}_{X}} \mathcal{O}_{W}=\mathcal{L}_{n} \otimes_{\mathcal{O}_{X}} \mathcal{O}_{W}$ for all $n \geqslant 0$. In other words $\mathcal{R} / \mathcal{J} \mathcal{R} \cong$ $\bigoplus_{n \geqslant 0}\left(\left.\mathcal{L}_{n}\right|_{W}\right) \bar{t}^{n}$, where $\bar{t}$ is now a formal symbol denoting the image of $t$. If we write $\overline{\mathcal{L}}=$ 
$\left.\mathcal{L}\right|_{W}$, then $\left.\mathcal{L}_{n}\right|_{W} \cong \overline{\mathcal{L}} \otimes_{\mathcal{O}_{W}} \overline{\mathcal{L}}^{\tau} \otimes \ldots \otimes \overline{\mathcal{L}}^{\tau^{n-1}}=\overline{\mathcal{L}}_{n}$ and so $\mathcal{R} / \mathcal{J} \mathcal{R}$ is just the bimodule algebra $\mathcal{B}\left(W,\left.\mathcal{L}\right|_{W},\left.\tau\right|_{W}\right)$, as defined in Section 8. Taking global sections we obtain

$$
\mathrm{H}^{0}(X, \mathcal{R} / \mathcal{J} \mathcal{R})=\bigoplus_{n \geqslant 0} \mathrm{H}^{0}\left(X, \mathcal{L}_{n} \otimes_{\mathcal{O}_{X}} \mathcal{O}_{W}\right) \bar{t}^{n}=\bigoplus_{n \geqslant 0} \mathrm{H}^{0}\left(W,\left.\mathcal{L}_{n}\right|_{W}\right) \bar{t}^{n}=B\left(W,\left.\mathcal{L}\right|_{W},\left.\tau\right|_{W}\right),
$$

where $B=B\left(W,\left.\mathcal{L}\right|_{W},\left.\tau\right|_{W}\right)$ is the ordinary twisted homogeneous coordinate ring.

Now take global sections of the short exact sequence of $\mathcal{O}_{X}$-modules

$$
0 \longrightarrow \mathcal{J R} \longrightarrow \mathcal{R} \longrightarrow \mathcal{R} / \mathcal{J} \mathcal{R} \longrightarrow 0
$$

to obtain the exact sequence of finitely generated right $R$-modules $0 \rightarrow N \rightarrow R \rightarrow B$, where $N=\mathrm{H}^{0}(X, \mathcal{J} \mathcal{R})$. Set

$$
T^{\prime}=\frac{T+N}{N}=\bigoplus_{n \geqslant 0} \bar{T}_{n}^{\prime} \bar{t}^{n} \subseteq R^{\prime}=\frac{R}{N} \subseteq B=B\left(W,\left.\mathcal{L}\right|_{W},\left.\tau\right|_{W}\right) .
$$

We claim that the hypotheses of the theorem pass to the pair of rings $T^{\prime} \subseteq B$ or, more precisely, that $T^{\prime} \subseteq B$ satisfy the analogous hypotheses of [3, Theorem 4.1]. To see this, observe first that $\bar{T}_{n}^{\prime}$ is the restriction to $W$ of the global sections contained in $\bar{T}_{n} \subseteq \bar{R}_{n}=\mathrm{H}^{0}\left(X, \mathcal{R}_{n}\right)$. Therefore, by condition (i) of the theorem, $\bar{T}_{n}^{\prime}$ generates the sheaf $\left.\mathcal{R}_{n}\right|_{W}=\left.\mathcal{L}_{n}\right|_{W}=\overline{\mathcal{L}}_{n}$ for all $n \gg 0$. Moreover, the rational map $\theta_{n}^{\prime}: W \rightarrow \mathbb{P}\left(\bar{T}_{n}^{\prime *}\right)$ is the restriction of the rational map $\theta_{n}: X \rightarrow \mathbb{P}\left(\bar{T}_{n}^{*}\right)$. Once again, as $W$ is $\tau$-invariant, $W \cap P_{n}=\emptyset$ for all $n$, and so, by condition (ii), $\theta_{n}^{\prime}$ is an everywhere-defined immersion. Since $W$ is proper, its image is closed and so $\theta_{n}^{\prime}$ is a closed immersion. Since $\mathcal{L}$ is $\tau$-ample, it follows from [15, Proposition 2.3(2) and Corollary 5.1] that $\left.\mathcal{L}\right|_{W}$ is both $\left.\tau\right|_{W}$-ample and $\left(\left.\tau\right|_{W}\right)^{-1}$-ample as an invertible sheaf over $W$. Moreover, by [15, Theorem 1.2(3)], $B$ has finite Gelfand-Kirillov dimension. The hypotheses of [3, Theorem 4.1] are therefore satisfied and, by that result, $B / T^{\prime}$ is finite dimensional. But (9.11) implies that $N_{n}=\mathrm{H}^{0}(X, \mathcal{J} \mathcal{R})_{n} \subseteq T_{n}$ for all $n \gg 0$ and so (9.12) implies that $R / T$ is also finite-dimensional.

Finally, $Z_{\mathcal{I}}$ is saturating by Lemma 9.5 .

In applications of Theorem 9.2 it is inconvenient to have to check conditions (i) and (ii) of that result for infinitely many choices of $n$. Fortunately, for rings generated in degree one, just one choice will do. In this final result we do not assume that Assumptions 9.1 are satisfied.

Corollary 9.13. Let $X$ be an integral projective scheme, with $\operatorname{dim} X \geqslant 2$, sheaf of rational functions $\mathcal{K}$ and with a $\tau$-ample invertible sheaf $\mathcal{L} \subset \mathcal{K}$ for some $\tau \in \operatorname{Aut}(X)$. Let $T=\bigoplus \bar{T}_{m} t^{m}$ be a noetherian cg subalgebra of $B(X, \mathcal{L}, \tau)=\bigoplus \mathrm{H}^{0}\left(X, \mathcal{L}_{m}\right) t^{m} \subseteq k(X)\left[t, t^{-1} ; \tau\right]$ that is generated in degree one. Let $P$ be a finite set of closed points in $X$ such that each $x \in P$ lies on a dense $\tau$-orbit. Finally, suppose that there exists $n \geqslant 1$ such that:

(i) $\bar{T}_{n}$ generates the sheaf $\mathcal{L}_{n}$, except, perhaps, at points of $P$.

(ii) The birational map $X \rightarrow \mathbb{P}\left(\bar{T}_{n}^{*}\right)$ defined by $\bar{T}_{n}$ defines, by restriction, an immersion $(X \backslash P) \hookrightarrow \mathbb{P}\left(\bar{T}_{n}^{*}\right)$.

Set $\mathcal{R}_{1}=\bar{T}_{1} \mathcal{O}_{X} \subseteq \mathcal{L}$ and let $\mathcal{I}=\mathcal{R}_{1} \mathcal{L}^{-1}$. Then $Z_{\mathcal{I}}$ is a saturating zero-dimensional subscheme of $X$ with $\operatorname{Supp} Z_{\mathcal{I}} \subseteq P$. Moreover $T \subseteq R=R\left(X, Z_{\mathcal{I}}, \mathcal{L}, \tau\right)$ with $\operatorname{dim}_{k} T / R<\infty$.

Proof. By definition, $\bar{T}_{1}$ generates the sheaf $\mathcal{R}_{1}=\mathcal{I} \mathcal{L}$. Since $T$ is generated in degree one, the space $\bar{T}_{m}=\bar{T}_{1} \bar{T}_{1}^{\tau} \ldots \bar{T}_{1}^{\tau^{m-1}}$ generates $\mathcal{R}_{m}=\mathcal{R}_{1} \mathcal{R}_{1}^{\tau} \ldots \mathcal{R}_{1}^{\tau^{m-1}}=\mathcal{I}_{m} \mathcal{L}_{m}$ for all $m \geqslant 1$. Thus we have the following inclusion of subrings of $k(X)\left[t, t^{-1} ; \tau\right]$ :

$$
T=\bigoplus_{m \geqslant 0} \bar{T}_{m} t^{m} \subseteq \bigoplus_{m \geqslant 0} \mathrm{H}^{0}\left(X, \mathcal{R}_{m}\right) t^{m}=R\left(X, Z_{\mathcal{I}}, \mathcal{L}, \tau\right)
$$


It is convenient to replace the integer $n$ by 1 in the statement of the corollary, which we will accomplish by passing to Veronese rings. Now the hypotheses of the corollary clearly pass across to the rings $T^{(n)} \subseteq B\left(X, \mathcal{L}_{n}, \tau^{n}\right)$ (in which case take $n=1$ in the statement of the result). Assume for the moment that the corollary holds for this pair of rings; thus Supp $Z_{\mathcal{I}_{n}} \subseteq P$ and $T^{(n)}$ is equal in large degree to $R\left(X, Z_{\mathcal{I}_{n}}, \mathcal{L}_{n}, \tau^{n}\right)$ or, in other words, $\bar{T}_{j n}=\mathrm{H}^{0}\left(X, \mathcal{R}_{j n}\right)$ for all $j \gg 0$. This ensures that Assumptions 9.1 also hold for these Veronese rings, and we can apply Lemma 9.3 with $V=\bar{T}_{a} \subseteq \mathrm{H}^{0}(X, \mathcal{F})$ where $\mathcal{F}=\mathcal{R}_{a}$ and $r=a$ for any $1 \leqslant a \leqslant n$. Together with Lemma 9.4, this shows that the multiplication map

$$
\bar{T}_{a} \otimes \bar{T}_{j n}^{\tau^{a}}=\bar{T}_{a} \otimes \mathrm{H}^{0}\left(X, \mathcal{R}_{j n}^{\tau^{a}}\right) \longrightarrow \mathrm{H}^{0}\left(X, \mathcal{R}_{a} \otimes \mathcal{R}_{j n}^{\tau^{a}}\right) \longrightarrow \mathrm{H}^{0}\left(X, \mathcal{R}_{j n+a}\right)
$$

is surjective for all $j \gg 0$. Thus $\bar{T}_{m}=\mathrm{H}^{0}\left(X, \mathcal{R}_{m}\right)$ for $m \gg 0$. Moreover, since we are assuming that $Z_{\mathcal{I}_{n}}$ is saturating and supported along $P$, clearly the same holds for $Z_{\mathcal{I}}$. Therefore, if the corollary holds for $T^{(n)} \subseteq B\left(X, \mathcal{L}_{n}, \tau^{n}\right)$, it also holds for $T \subseteq B(X, \mathcal{L}, \tau)$.

Thus we have reduced to the case $n=1$ and we aim to show that the hypotheses (i) and (ii) of Theorem 9.2 are satisfied for all $m \geqslant 1$. We have already seen that $\bar{T}_{m}$ generates $\mathcal{R}_{m}$ for all $m \geqslant 1$, so hypothesis (i) of the theorem holds. Set $P_{1}=P$ and, for $m \geqslant 1$, define $P_{m}=\bigcup_{i=0}^{m-1} \tau^{-i}\left(P_{1}\right) \supseteq \operatorname{Supp} \mathcal{O}_{X} / \mathcal{I}_{m}$. Note that $\bar{T}_{1}$ generates $\mathcal{L}$ along $X \backslash P_{1}$. For any $j \in \mathbb{Z}$, condition (ii) implies that the space $\bar{T}(j)=\bar{T}_{1}^{\tau^{j}}$ defines an immersion $X \backslash P_{n} \hookrightarrow X \backslash \tau^{-j}\left(P_{1}\right) \hookrightarrow$ $\mathbb{P}\left(\bar{T}(j)^{*}\right)$, and so an easy induction using Lemma 7.3 shows that $\bar{T}_{m}$ defines an immersion $\left(X \backslash P_{m}\right) \hookrightarrow \mathbb{P}\left(\bar{T}_{m}^{*}\right)$ for all $m \geqslant 1$. Thus hypothesis (ii), and hence all the hypotheses, of Theorem 9.2 are verified and the corollary follows.

\section{The main theorem}

We can now put everything together to prove the main theorem of the paper, which is an elaboration of Theorem 1.1.

Theorem 10.1. Let $A=\bigoplus_{i \geqslant 0} A_{i}$ be a cg noetherian domain that is generated in degree one and birationally 2-geometric. Then we have the following.

(i) There exists a naïve blowup algebra $R=R\left(\mathbb{X}, Z_{\mathcal{I}}, \mathcal{L}, \widetilde{\sigma}\right)$, where $Z_{\mathcal{I}}$ is a (possibly empty) saturating zero-dimensional subscheme of $\mathbb{X}$ such that $A \subseteq R$ with $\operatorname{dim}_{k} R / A<\infty$.

(ii) The data $\left\{\mathbb{X}, Z_{\mathcal{I}}, \mathcal{L}, \widetilde{\sigma}\right\}$ can be made more precise as follows.

(a) for any stable value of $n$, the scheme $\mathbb{X}=\mathbb{X}_{n}$ is the blow down $\pi_{n}\left(Y_{n}\right)$ of the relevant component $Y_{n}$ of the truncated point scheme, as constructed by Theorem 7.1;

(b) the automorphism $\widetilde{\sigma}=\pi_{n} \sigma \pi_{n}^{-1}$ of $\mathbb{X}$ is induced from the birational map $\sigma_{n}=\psi_{n} \phi_{n}^{-1}$ of $Y_{n}$;

(c) if one writes $A=\bigoplus \bar{A}_{n} t^{n} \subset Q(A)=k(\mathbb{X})\left[t, t^{-1}, \sigma\right]$ for some $0 \neq t \in A_{1}$, then $\mathcal{L}=$ $\left(\bar{A}_{1} \mathcal{O}_{\mathbb{X}}\right)^{* *}$ with $\bar{A}_{1} \mathcal{O}_{\mathbb{X}}=\mathcal{I} \mathcal{L}$.

Before proving the theorem we need some subsidiary results and we begin by setting up the notation that will remain in force throughout the section. Fix a stable value of $n$, let $Y_{n}$ denote the relevant component of $W_{n}$ and drop the subscript from $Y=Y_{n}$, etc. Let $\pi: Y \rightarrow \mathbb{X}$ be the birational surjective morphism defined by Theorem 7.1. Recall from that result that $\widetilde{\sigma}=\pi \sigma \pi^{-1}$ is a biregular automorphism of $\mathbb{X}$, and that the set of points where $\pi^{-1}$ is not defined is a finite set of nonsingular points $P$, each lying on a dense $\widetilde{\sigma}$-orbit. Set $V=\mathbb{X} \backslash P$ and $U=\pi^{-1}(V) \subseteq Y$; thus $U \cong V$. As in the statement of the theorem, fix $t \in A_{1} \backslash\{0\}$ and write $A_{i}=\bar{A}_{i} t^{i}$ for $i \geqslant 0$; thus each $\bar{A}_{i} \subset K=Q(A)_{0}$ and $A=\bigoplus \bar{A}_{n} t^{n} \subset Q(A)=K\left[t, t^{-1} ; \sigma\right]$. We recall that $K \cong k(Y)=k(\mathbb{X})$ by Corollary and we always write the corresponding sheaf of rational functions (on $Y$ or $\mathbb{X}$ ) as $\mathcal{K}$. 
We begin with some elementary observations about invertible sheaves.

Lemma 10.2. Let $\mathcal{A}=\sum_{i=1}^{a} s_{i} \mathcal{O}_{Y} \subset \mathcal{K}$ be a sheaf generated by sections $s_{j} \in \mathrm{H}^{0}(Y, \mathcal{A})$ and set $\mathcal{B}=\pi_{*} \mathcal{A} \subset \mathcal{K}$ with reflexive hull $\mathcal{P}=\mathcal{B}^{* *}$. If $\mathcal{A}(u)=\sum_{i=1}^{a} s_{i}^{\sigma^{u-1}} \mathcal{O}_{Y}$ is invertible for $1 \leqslant u \leqslant v$, then $\mathcal{B}(u)=\pi_{*} \mathcal{A}(u)$ has reflexive hull $\mathcal{P}(u)=\mathcal{P}^{\tilde{\sigma}^{u-1}}$ for $1 \leqslant u \leqslant v$. Moreover, $\mathcal{P}(1)$. $\ldots \cdot \mathcal{P}(v)$ is the reflexive hull of $\pi_{*}(\mathcal{A}(1) \cdot \ldots \cdot \mathcal{A}(v))$.

Proof. As $\widetilde{\sigma}$ and $\sigma$ induce the same automorphism of $K$, we have $s_{i}^{\widetilde{\sigma}^{v}}=s_{i}^{\sigma^{v}}$ for all $i, v$. Let $\tau=\widetilde{\sigma}^{u-1}$ for some $1 \leqslant u \leqslant v$. Since $\left.\mathcal{P}\right|_{V}=\sum s_{i} \mathcal{O}_{V}$ we have $\left.\mathcal{P}^{\tau}\right|_{\tau^{-1}(V)}=\sum s_{i}^{\tau} \mathcal{O}_{\tau^{-1}(V)}$. Set $V^{\prime}=V \cap \tau^{-1}(V)$ and note that $\mathbb{X} \backslash V^{\prime}$ consists of a finite set of closed nonsingular points of $Y$. Since

$$
\left.\mathcal{B}(u)\right|_{V^{\prime}}=\pi_{*}\left(\left.\mathcal{A}(u)\right|_{\pi^{-1}\left(V^{\prime}\right)}\right)=\sum_{i=1}^{a} s_{i}^{\tau} \mathcal{O}_{V^{\prime}}=\left.\mathcal{P}^{\tau}\right|_{V^{\prime}},
$$

Sublemma 7.7(ii) implies that $\mathcal{P}^{\tau}=\mathcal{B}(u)^{* *}$. The proof of the last assertion is similar.

Lemma 10.3. (i) The scheme $\mathbb{X}$ has a $\widetilde{\sigma}$-ample invertible sheaf.

(ii) Let $\mathcal{P}$ be an invertible sheaf on $\mathbb{X}$, and write $\mathcal{P}_{\ell}=\mathcal{P} \otimes \mathcal{P}^{\tilde{\sigma}} \otimes \ldots \otimes \mathcal{P}^{\tilde{\sigma}^{\ell-1}}$ for $\ell \geqslant 1$. If $\mathcal{P}_{n} \otimes \mathcal{P}_{n}^{\tilde{\sigma}^{q}}$ is ample for some $n \geqslant 1$ and all $q \gg 0$ then $\mathcal{P}$ is $\widetilde{\sigma}$-ample.

Proof. (i) As $A$ is noetherian, [27, Theorem 0.1] implies that $A$ has sub-exponential growth. Since $Q(A)=K\left[t, t^{-1} ; \sigma\right],[\mathbf{2 4}$, Proposition 3.5 and Lemma 2.3(2)] together with $[\mathbf{1 5}$, Theorem 1.2(2)] imply that $\mathbb{X}$ has a $\widetilde{\sigma}$-ample invertible sheaf.

(ii) By part (i) and [15, Theorem 1.3], $\widetilde{\sigma}$ is quasi-unipotent in the sense of [15]. By hypothesis, $\mathcal{F}=\mathcal{P}_{n} \otimes \mathcal{P}_{n}^{\tilde{\sigma}^{m n}}$ is ample for some $m \geqslant 1$. Hence, so is $\mathcal{G}=\mathcal{F} \otimes \mathcal{F}^{\tilde{\sigma}^{n}} \otimes \cdots \otimes \mathcal{F}^{\widetilde{\sigma}^{n m-n}}$. But expanding terms, one finds that $\mathcal{G}=\mathcal{P}_{2 n m}$. By [15, Theorem 1.3], $\mathcal{P}$ is therefore $\widetilde{\sigma}$-ample.

Proof of Theorem 10.1. We continue to use the notation set up immediately after the statement of the theorem. The aim of the proof is to apply Corollary 9.13 and this will be easy to do once we understand the relationship between sheaves of $\mathcal{O}_{Y}$-modules generated by the $\bar{A}_{j}$ and those induced from the embedding of $Y$ into $\mathbb{P}\left(A_{1}^{*}\right)$. Thus to begin, we want to be very precise about the maps of schemes involved, after which we will translate the resulting identifications into ones involving sheaves.

Fix a basis $\left\{x_{j}=a_{j} t: 0 \leqslant j \leqslant r\right\}$ of $A_{1}$, where each $a_{j} \in \bar{A}_{1} \subset K$. Write $\mathbb{P}=\mathbb{P}\left(A_{1}^{*}\right)$ and let $\iota: Y \hookrightarrow W \hookrightarrow \mathbb{P}^{\times n}$ denote the restriction to $Y$ of the natural inclusion described in Section 2 . For $1 \leqslant m \leqslant n$, let $\rho_{m}: \mathbb{P}^{\times n} \rightarrow \mathbb{P}$ be the projection onto the $m$ th copy of $\mathbb{P}$. As usual, we regard $Q(A)_{\geqslant 0}$ as a 'generic' right $K$-point module, where $K$ acts by left multiplication. By Corollary 3.5, the truncated $K$-point module $\bigoplus_{i=0}^{n} Q(A)_{i}$ corresponds to a map $\theta: \eta=$ Spec $K \rightarrow Y$ and hence, for $1 \leqslant m \leqslant n$, induces a map

$$
\chi_{m}: \eta \stackrel{\theta}{\longrightarrow} Y \stackrel{\iota}{\longrightarrow} \mathbb{P}^{\times n} \stackrel{\rho_{m}}{\longrightarrow} \mathbb{P} .
$$

As in Notation 5.2, we identify $K=k(Y)$ through $\theta$.

We need the precise form of $\theta$, so we recall its construction from [4]. Write $\underline{a}=\left(a_{0}: a_{1}\right.$ : $\left.\ldots: a_{r}\right)$, regarded as a $K$-valued point of $\mathbb{P}$. The decomposition $\bigoplus_{i=0}^{n} Q(A)_{i}=\bigoplus_{i=0}^{n} K t^{i}$ fixes the generator $t^{i}$ of the free $K$-module $Q(A)_{i}$ and the right action of $A_{1}$ is then given by the formula $t^{i} x_{j}=a_{j}^{\sigma^{i}} t^{i+1}$ for all $i, j \geqslant 0$. Thus, by the proof of [4, Proposition 3.9] (see [4, Equations 3.10 and 3.11] in particular), the map $\iota \theta$ associates to $\eta$ the $K$-valued point $\left(\underline{a}, \underline{a}^{\sigma}, \ldots, \underline{a}^{\sigma^{n-1}}\right)$ of $\mathbb{P}^{\times n}$. Equivalently, for $1 \leqslant m \leqslant n, \chi_{m}$ associates $\eta$ to the $K$-valued 
point $\underline{a}^{\sigma^{m-1}}$ of $\mathbb{P}$. Alternatively, regard the $\left\{x_{j}\right\}$ as coordinate functions on $\mathbb{P}$ and set $U_{j}=\left\{p \in \mathbb{P}: x_{j}(p) \neq 0\right\}$ for $0 \leqslant j \leqslant r$. Then, for $0 \leqslant j \leqslant r$ and $1 \leqslant \ell \leqslant n$, the map of rings $\mathcal{O}\left(U_{j}\right)=k\left[x_{1} x_{j}^{-1}, \ldots, x_{r} x_{j}^{-1}\right] \rightarrow K$ induced by $\chi_{\ell}$ is simply defined by $x_{i} x_{j}^{-1} \mapsto s_{i}(\ell) s_{j}(\ell)^{-1}$ for $s_{m}(\ell)=a_{m}^{\sigma^{\ell-1}}$.

We now use this information to construct invertible sheaves on $Y$, which we do by following the proof of [14, Theorem II.7.1]. Fix $1 \leqslant m \leqslant n$ and let $P(m)=K$, regarded as a locally free sheaf on $\eta=\operatorname{Spec} K$ generated by the sections $\left\{s_{i}(m): 0 \leqslant i \leqslant r\right\}$. By the proof of $[\mathbf{1 4}$, Theorem II.7.1(b)], this data defines a morphism $\chi_{m}^{\prime}: \eta \rightarrow \mathbb{P}$ given by $x_{i} x_{j}^{-1} \mapsto s_{i}(m) s_{j}(m)^{-1}$ as a homomorphism $\mathcal{O}\left(U_{j}\right) \rightarrow K$; in other words it defines the morphism $\chi_{m}$. Conversely, by $\left[\mathbf{1 4}\right.$, Theorem II.7.1(a)], we can identify $P(m)=\chi_{m}^{*} \mathcal{O}_{\mathbb{P}}(1)$ via the explicit formula $s_{i}(m)=$ $\chi_{m}^{*}\left(x_{i}\right)$ for $0 \leqslant i \leqslant r$.

Now apply [14, Theorem II.7.1(a)] to the map $\widetilde{\chi}_{m}=\rho_{m} \iota: Y \rightarrow \mathbb{P}$ for some $1 \leqslant m \leqslant n$. Since $\mathcal{O}_{Y}$ is embedded into $\mathcal{K}$ via $\theta$, we have $\tilde{\chi}_{m}^{*}\left(x_{i}\right)=\chi_{m}^{*}\left(x_{i}\right)=s_{i}(m)$ for each $i$. Therefore, $[\mathbf{1 4}$, Theorem II.7.1(a)] shows that the elements $\left\{s_{i}(m): 0 \leqslant i \leqslant r\right\}$ generate a locally free sheaf of $\mathcal{O}_{Y}$-modules $\mathcal{N}(m) \cong \tilde{\chi}_{m}^{*}\left(\mathcal{O}_{\mathbb{P}}(1)\right)=\iota^{*} \rho_{m}^{*}\left(\mathcal{O}_{\mathbb{P}}(1)\right)$.

To summarize, as $\left\{a_{i}^{\sigma^{m-1}}: 0 \leqslant i \leqslant r\right\}$ is a $k$-basis of $\bar{A}_{1}^{\sigma^{m-1}}$, we have shown:

$$
\bar{A}_{1}^{\sigma^{m-1}} \subseteq \mathrm{H}^{0}(Y, \mathcal{N}(m)) \subseteq K \text { and } \mathcal{N}(m)=\bar{A}_{1}^{\sigma^{m-1}} \mathcal{O}_{Y} \subseteq \mathcal{K} \quad \text { for } 1 \leqslant m \leqslant n .
$$

Now consider the invertible sheaf of $\mathbb{P}^{\times n}$-modules

$$
\mathcal{O}(1, \ldots, 1)=\rho_{1}^{*} \mathcal{O}_{\mathbb{P}}(1) \otimes \rho_{2}^{*} \mathcal{O}_{\mathbb{P}}(1) \otimes \ldots \otimes \rho_{n}^{*} \mathcal{O}_{\mathbb{P}}(1),
$$

where the tensor products are over $\mathcal{O}_{\mathbb{P} \times n}$. Since pull-backs commute with tensor products, and the tensor product of the $\mathcal{N}(m)$ is isomorphic to their product inside $\mathcal{K}$, it follows that $\mathcal{M}=\mathcal{N}(1) \mathcal{N}(2) \cdot \ldots \cdot \mathcal{N}(n)$ satisfies

$$
\mathcal{M} \cong \iota^{*} \rho_{1}^{*} \mathcal{O}_{\mathbb{P}}(1) \otimes \ldots \otimes \iota^{*} \rho_{n}^{*} \mathcal{O}_{\mathbb{P}}(1)=\iota^{*} \mathcal{O}(1, \ldots, 1) .
$$

Therefore, by (10.5),

$$
\bar{A}_{n}=\bar{A}_{1} \bar{A}_{1}^{\sigma} \cdot \ldots \cdot \bar{A}_{1}^{\sigma^{n-1}} \subseteq \mathrm{H}^{0}(Y, \mathcal{N}(1)) \cdot \ldots \cdot \mathrm{H}^{0}(Y, \mathcal{N}(n)) \subseteq \mathrm{H}^{0}(Y, \mathcal{M}),
$$

where $\mathcal{M}=\bar{A}_{n} \mathcal{O}_{Y}$. Since

$$
\mathrm{H}^{0}\left(\mathbb{P}^{\times n}, \mathcal{O}(1, \ldots, 1)\right)=\mathrm{H}^{0}\left(\mathbb{P}^{\times n}, \rho_{1}^{*} \mathcal{O}_{\mathbb{P}}(1)\right) \otimes_{k} \ldots \otimes_{k} \mathrm{H}^{0}\left(\mathbb{P}^{\times n}, \rho_{n}^{*} \mathcal{O}_{\mathbb{P}}(1)\right),
$$

it follows that $\bar{A}_{n}$ is just the image of the natural map

$$
\iota^{*}: \mathrm{H}^{0}\left(\mathbb{P}^{\times n}, \mathcal{O}(1,1, \ldots, 1)\right) \longrightarrow \mathrm{H}^{0}\left(Y, \iota^{*}(\mathcal{O}(1,1, \ldots, 1))=\mathrm{H}^{0}(Y, \mathcal{M}) .\right.
$$

On the other hand, $\mathcal{O}(1,1, \ldots, 1)$ is a very ample invertible sheaf over $\mathbb{P}^{\times n}$ and so its set of global sections $N=\mathrm{H}^{0}\left(\mathbb{P}^{\times n}, \mathcal{O}(1, \ldots, 1)\right)$ defines a closed immersion $\xi: \mathbb{P}^{\times n} \hookrightarrow \mathbb{P}\left(N^{*}\right)$. By restriction, $\xi$ defines a closed immersion of $Y$ into projective space; to be precise, $\bar{N}=\iota^{*}(N)$ defines a closed immersion of $Y$ into $\mathbb{P}\left(\bar{N}^{*}\right) \hookrightarrow \mathbb{P}\left(N^{*}\right)$. Comparing this with the conclusion of the previous paragraph we deduce:

$$
\begin{aligned}
& \mathcal{M} \text { is invertible and the sections } \bar{A}_{n} \subseteq \mathrm{H}^{0}(Y, \mathcal{M}) \\
& \text { define a closed immersion } Y \hookrightarrow \mathbb{P}\left(\bar{A}_{n}^{*}\right) .
\end{aligned}
$$

We now push everything forward to $\mathbb{X}=\mathbb{X}_{n}$ via the map $\pi: Y \rightarrow \mathbb{X}$ constructed in Theorem 7.1. Recall that $V=\mathbb{X} \backslash P$ and that $\pi^{-1}: V \rightarrow U=\pi^{-1}(V)$ is an isomorphism. For each $m$, write $\mathcal{F}(m)=\pi_{*} \mathcal{N}(m)$, with reflexive hull $\mathcal{L}(m)=\mathcal{F}(m)^{* *}$. By Sublemma 7.7(i), each $\mathcal{L}(m)$ is an invertible sheaf of $\mathcal{O}_{\mathbb{X}}$-modules contained in $\mathcal{K}$. Moreover, Lemma 10.2 and (10.5) imply that $\mathcal{L}(m)=\mathcal{L}(1)^{\tilde{\sigma}^{m-1}}$ for $1 \leqslant m \leqslant n$ and that $\mathcal{L}(1) \mathcal{L}(2) \cdot \ldots \cdot \mathcal{L}(n)=\left(\pi_{*} \mathcal{M}\right)^{* *}$. In other words, $\left(\pi_{*} \mathcal{M}\right)^{* *}=\mathcal{L}_{n}=\mathcal{L} \mathcal{L}^{\tilde{\sigma}} \cdot \ldots \cdot \mathcal{L}^{\tilde{\sigma}^{n-1}}$ for $\mathcal{L}=\mathcal{L}(1) \subset \mathcal{K}$. 
By (10.7), $\mathcal{M}$ is a very ample invertible sheaf on $Y$ while $\mathcal{L}_{n}=\left(\pi_{*} \mathcal{M}\right)^{* *}$ by definition. Thus, Remark 7.8 implies that $\mathcal{L}_{n} \otimes\left(\mathcal{L}_{n}\right)^{\tilde{\sigma}^{q}}$ is ample for all $q \gg 0$. Hence, by Lemma $10.3(\mathrm{ii})$ and the previous paragraph, $\mathcal{L}$ is a $\widetilde{\sigma}$-ample invertible sheaf.

We are now ready to check the hypotheses of Corollary 9.13. By its construction in Theorem 7.1, $\widetilde{\sigma}$ induces the automorphism $\sigma$ of $K$. Since $\mathcal{L} \subset \mathcal{K}$ this implies that $B(\mathbb{X}, \mathcal{L}, \widetilde{\sigma})=$ $\bigoplus_{n \geqslant 0} \mathrm{H}^{0}\left(\mathbb{X}, \mathcal{L}_{n}\right) t^{n} \subset K[t ; \sigma]$. As $A$ is generated in degree one by $A_{1}=\bar{A}_{1} t \subseteq \mathrm{H}^{0}(\mathbb{X}, \mathcal{L}) t$, it follows that $A$ is a subring of $B(\mathbb{X}, \mathcal{L}, \widetilde{\sigma})$, and hence $\bar{A}_{n} t^{n} \subseteq \mathrm{H}^{0}\left(\mathbb{X}, \mathcal{L}_{n}\right) t^{n}$. Since $\mathcal{L}$ is a $\widetilde{\sigma}$-ample invertible sheaf by the previous paragraph, and $P$ consists of a finite set of points lying on dense $\widetilde{\sigma}$-orbits by Theorem 7.1, the hypotheses from the first three sentences of Corollary 9.13 are satisfied in the present setup. By $(10.6),\left.\mathcal{L}_{n}\right|_{V}=\left.\pi_{*} \mathcal{M}\right|_{V}=\left.\mathcal{M}\right|_{U}$ is generated by $\bar{A}_{n}$ and so condition (i) of the corollary holds, while condition (ii) of the corollary follows from (10.7). We can therefore apply the corollary to find a saturating zero-dimensional subscheme $Z_{\mathcal{I}} \subset \mathbb{X}$ such that $A \subseteq R=R\left(\mathbb{X}, Z_{\mathcal{I}}, \mathcal{L}, \widetilde{\sigma}\right)$, with $\operatorname{dim}_{k} R / A<\infty$. This proves part (i) of the theorem.

Finally, parts (iia) and (iib) of the theorem both follow from Theorem 7.1 together with our construction of $\mathbb{X}$. Corollary 9.13 also ensures that $\mathcal{I}$ is defined by $\mathcal{L I}=\bar{A}_{1} \mathcal{O}_{\mathbb{X}}$, while $(10.5)$ shows that $\mathcal{L}=\left(\pi_{*} \mathcal{M}\right)^{* *}=\left(\bar{A}_{1} \mathcal{O}_{\mathbb{X}}\right)^{* *}$. Thus part (iic) of the theorem holds.

As an application of the theorem, we get the following criterion for a noetherian cg domain to be a twisted homogeneous coordinate ring. Up to changes in phraseology (the set $S_{\infty}^{+}$does not appear in [24]) this result is also a special case of [24, Theorem 4.4].

Corollary 10.8. Let $A$ be a noetherian $\mathrm{cg}$ domain that is generated in degree one and birationally 2-geometric. Suppose that the set of extremal elements $S_{\infty}^{+}$, as defined in (5.6), is empty. Then, up to a finite-dimensional vector space, $A \cong B(\mathbb{X}, \mathcal{L}, \sigma)$, where $\mathbb{X}=Y_{n}$ for any stable value of $n$.

Proof. The first paragraph of the proof of Theorem 7.1 shows that $\mathbb{X}=Y=Y_{n}$. Therefore, the set $P$ constructed in the proof of Theorem 10.1 is also empty and so Corollary 9.13 , or indeed [3, Theorem 4.1], shows that $A \cong R(\mathbb{X}, \emptyset, \mathcal{L}, \sigma)=B(Y, \mathcal{L}, \sigma)$ up to a finite-dimensional vector space.

Finally, observe that if $\sigma$ is an automorphism of a field $D$ then $D\left[t, t^{-1} ; \sigma\right]$ satisfies a polynomial identity if and only if $|\sigma|<\infty$. Since non-empty saturating sets only exist when $|\sigma|=\infty$, we obtain the following special case of Theorem 10.1.

Corollary 10.9. Let $A$ be a $\mathrm{cg}$ noetherian domain that is generated in degree one and birationally 2-geometric. If $A$ satisfies a polynomial identity then there exists a twisted homogeneous coordinate ring $B=B(\mathbb{X}, \mathcal{L}, \sigma)$ such that $A \subseteq B$ with $\operatorname{dim}_{k} B / A<\infty$.

\section{Examples}

In this section, we give two examples which illustrate the earlier results in the paper. The first example shows that the truncated point schemes $W_{n}$ and the relevant components $Y_{n}$ are, in general, distinct; indeed the dimension can differ by arbitrarily large amounts. Surprisingly, this happens when one takes the naïve blowup algebra at a high power of a sheaf of maximal ideals.

EXAmPle 11.1. Let $X$ be an integral projective scheme with $\operatorname{dim} X \geqslant 2$ and $\sigma \in$ Aut $X$ for which there exists a very ample and $\sigma$-ample invertible sheaf $\mathcal{L}$ and a point $c \in X$ such 
that the orbit $\langle\sigma\rangle \cdot c$ is critically dense. Write $\mathcal{I}=\mathcal{I}_{c}$ for the ideal sheaf of the point $c$. Then, for any integer $t \geqslant 1$ there exist $p, q \in \mathbb{N}$ with the following properties.

(i) The ring $R=R\left(X, Z_{\mathcal{I}^{p}}, \mathcal{L}^{q}, \sigma\right)$ is noetherian and generated in degree one.

(ii) For any $n \geqslant 1$ the truncated point scheme $W_{n}$ for $R$ satisfies $\operatorname{dim} W_{n} \geqslant t$.

(iii) For any $n \geqslant 1$ there exists a point $w \in W_{n}$ such that the fibre $\phi_{n}^{-1}(w)$ has dimension at least $t$.

Remarks 11.2. (i) Note that, if $X$ is a surface in this example and $n$ is a stable value then $\operatorname{dim} Y_{n}=2$, by Corollary 3.5. Therefore, if $t>2$, then $W_{n} \neq Y_{n}$ for all $n \gg 0$.

(ii) An explicit example can be obtained by using the data from Section 1.2; thus, modulo a slight change of notation, take $X=\mathbb{P}^{2}$ with $\mathcal{L}=\mathcal{O}(1), c=(1: 1: 1)$ and $\sigma$ defined by $\left(\lambda_{0}: \lambda_{1}\right.$ : $\left.\lambda_{2}\right) \mapsto\left(\lambda_{0}: \alpha \lambda_{1}: \beta \lambda_{2}\right)$, where $\alpha, \beta \in k^{*}$ are algebraically independent over the prime subfield of $k$.

(iii) This result is in striking contrast to the commutative case, where blowing up at a maximal ideal $\mathcal{I}$ and at some power $\mathcal{I}^{p}$ of $\mathcal{O}_{X}$ gives the same scheme [14, Exercise II.7.11].

Proof. In the proof, tensor products will be taken over $\mathcal{O}_{X}$. As $\operatorname{dim} X \geqslant 2$ we can pick $p$ such that length $\mathcal{I}^{p} / \mathcal{I}^{p+1} \geqslant t+1$. Set $\mathcal{J}=\mathcal{I}^{p}$. We want to pick $q$ so that there are no complications arising from cohomology. Explicitly, by [23, Proposition 3.20 and Corollary 3.21] we can choose $q \geqslant 1$ such that:

(i) the ring $R\left(X, Z_{\mathcal{J}}, \mathcal{L}^{q}, \sigma\right)$ is generated in degree one, and

(ii) $\mathrm{H}^{1}\left(X, \mathcal{I}^{\sigma^{u}} \otimes \mathcal{J}_{a} \otimes \mathcal{L}_{a}^{q}\right)=0$, for all $u \in \mathbb{Z}$ and $a \geqslant 1$.

For these values of $p$ and $q$ set $R=R\left(X, Z_{\mathcal{J}}, \mathcal{L}^{q}, \sigma\right)$ and $\mathcal{R}=\mathcal{R}\left(X, Z_{\mathcal{J}}, \mathcal{L}^{q}, \sigma\right)$. The ring $R$ is noetherian by Proposition 8.7(ii) and so (i) holds. Let $\mathcal{H}=\mathcal{I}^{\sigma^{n}}$ for some $n \geqslant 1$. The point of the proof will be to find a $t$-dimensional family of truncated point modules of length $n+2$ among the quotients of the $R$-module $M=\mathrm{H}^{0}(X, \mathcal{R} / \mathcal{H} \mathcal{R})$.

Taking cohomology of the exact sequence $0 \rightarrow \mathcal{H} \mathcal{R} \rightarrow \mathcal{R} \rightarrow \mathcal{R} / \mathcal{H} \mathcal{R} \rightarrow 0$ gives the exact sequence of $R$-modules

$$
0 \longrightarrow \mathrm{H}^{0}(X, \mathcal{H} \mathcal{R}) \longrightarrow R \stackrel{\chi}{\longrightarrow} M \longrightarrow \bigoplus_{a=0}^{\infty} \mathrm{H}^{1}\left(X, \mathcal{H} \mathcal{J}_{a} \otimes \mathcal{L}_{a}^{q}\right)
$$

Suppose first that $a \geqslant 1$; thus $\mathrm{H}^{1}\left(X, \mathcal{H} \otimes \mathcal{J}_{a} \otimes \mathcal{L}_{a}^{q}\right)=0$, by the choice of $q$. Since the kernel $\mathcal{D}$ of the natural surjection $\mathcal{H} \otimes \mathcal{J}_{a} \otimes \mathcal{L}_{a}^{q} \rightarrow \mathcal{H} \mathcal{J}_{a} \otimes \mathcal{L}_{a}^{q}$ is supported at the point $\sigma^{-n}(c)$, certainly $\mathrm{H}^{2}(X, \mathcal{D})=0$. Combining these observations shows that $\mathrm{H}^{1}\left(X, \mathcal{H J}_{a} \otimes \mathcal{L}_{a}^{q}\right)=0$ as well and so $\chi_{a}: R_{a} \rightarrow M_{a}$ is surjective for all $a \geqslant 1$. When $a=0, R_{0}=M_{0}=k$ and $\mathrm{H}^{0}\left(X, \mathcal{H} \mathcal{R}_{0}\right)=$ $\mathrm{H}^{0}(X, \mathcal{H})=0$. Thus $\chi_{0}$ is an isomorphism, $\chi$ is surjective and $M$ is cyclic.

For any $r \geqslant 0$,

$$
M_{r}=\mathrm{H}^{0}\left(X, \frac{\mathcal{J}_{r} \otimes \mathcal{L}_{r}^{q}}{\mathcal{H} \mathcal{J}_{r} \otimes \mathcal{L}_{r}^{q}}\right) \cong \mathrm{H}^{0}\left(X, \frac{\mathcal{J}_{r}}{\mathcal{H} \mathcal{J}_{r}}\right)
$$

If $r \leqslant n$ then $\mathcal{H}=\mathcal{I}^{\sigma^{n}}$ and $\mathcal{J}_{r}=\mathcal{J} \cdot \ldots \cdot \mathcal{J}^{\sigma^{r-1}}$ are comaximal and so $M_{r} \cong \mathrm{H}^{0}\left(X, \mathcal{O}_{X} / \mathcal{H}\right) \cong$ $k$. However,

$$
M_{n+1} \cong \mathrm{H}^{0}\left(X, \frac{\mathcal{J} \cdot \ldots \cdot \mathcal{J}^{\sigma^{n}}}{\mathcal{H} \mathcal{J} \cdot \ldots \cdot \mathcal{J}^{\sigma^{n}}}\right) \cong \mathrm{H}^{0}\left(X, \frac{\mathcal{H}^{p}}{\mathcal{H}^{p+1}}\right)
$$

This has dimension $T \geqslant t+1$ by the choice of $p$. For each $(T-1)$-dimensional subspace $V \subset$ $M_{n+1}$, let $M(V)=M /\left(V R+M_{n+2} R\right) \cong R / Q(V)$, say. By the above analysis, this is a cyclic $R$-module with $\operatorname{dim}_{k} M(V)_{j}=1$ for $0 \leqslant j \leqslant n+1$; in other words $M(V)$ is a truncated point module of length $n+2$. Now, truncated point modules $R / Q$ and $R / Q^{\prime}$ are isomorphic if and only if $Q=Q^{\prime}$. Since $Q\left(V_{1}\right) \neq Q\left(V_{2}\right)$ if $V_{1} \neq V_{2}$, we have therefore constructed a family of 
truncated point modules of length $n+2$ parametrized by $\mathbb{P}\left(M_{n+1}^{*}\right)$. Thus $\operatorname{dim} W_{n+1} \geqslant t$ for all $n \geqslant 0$, proving (ii). Finally, each of the modules $M(V)$ has the same truncation $M / M_{n+1} R$ and so, by Lemma 2.3(i), the fibre of $\phi_{n}$ over the point $w \in W_{n}$ corresponding to $M / M_{n+1} R$ also has dimension at least $t$.

In order to illustrate the questions raised in Section 1.5, we end by giving an example of a (nonnoetherian) cg domain $A$ that is birationally commutative but not birationally 2-geometric. As mentioned there, we conjecture that examples of this type are always nonnoetherian.

EXAmple 11.3. Let $K=k(u, v)$ be a rational function field over $k$, and define $\sigma: K \rightarrow K$ by $u \mapsto u, v \mapsto u v$. Let $Q=K\left[z, z^{-1} ; \sigma\right]$ and $A=k\langle u z, v z, z\rangle \subset Q$. Then $A$ is a nonnoetherian domain that is birationally commutative but not birationally 2 -geometric.

Proof. It is a routine exercise, which we leave to the reader, to show that GKdim $A=4$ (mimic the proof of [18, Example 8.2.16]). Thus $A$ is an Ore domain by [18, Corollary 8.1.21] and since $u, v \in A_{1} A_{1}^{-1}$ it is clear that $Q$ is the graded ring of fractions of $A$.

In order to prove that $A$ is not noetherian, write $f_{n}=(v z) z^{n}=v z^{n+1}$ for $n \geqslant 0$ and let $I^{(n)}=\sum_{i=0}^{n} A f_{i}$. Note that any element $a \in A_{r}$ has the form $a=q z^{r}$, for some $q \in k[u, v]$. Hence, if $r>0$ then $a f_{n-r}=q z^{r} v z^{n-r+1}=q u^{r} v z^{n+1} \in k[u, v] u f_{n}$. Thus, $f_{n} \notin I^{(n-1)}$ for $n \geqslant 1$ and $I^{(0)} \subsetneq I^{(1)} \subsetneq \ldots$ gives a proper ascending chain of left ideals of $A$.

Clearly $A$ is birationally commutative. There are several ways to see that $A$ is not birationally 2-geometric. First of all, [10, Remark 7.3] shows that there is no projective model $Y$ of $K=k(u, v)$ with an automorphism inducing the given $\sigma \in \operatorname{Aut}(K)$, so $A$ is not birationally 2 -geometric by definition. Alternatively if $A$ were birationally 2-geometric, then $[\mathbf{2 4}$, Theorem 1.6] would imply that $\operatorname{GKdim}(A)=\operatorname{GKdim}(B)$ for some twisted homogeneous coordinate $\operatorname{ring} B(Y, \mathcal{L}, \sigma)$ with $Q(B)=K\left[t, t^{-1} ; \sigma\right]$, where $Y$ would necessarily be a surface and $\mathcal{L}$ would be $\sigma$-ample. But, by [6, Theorem 1.7], no such twisted homogeneous coordinate ring has GK-dimension 4.

Acknowledgements. We thank James Zhang, Mike Artin and Johan de Jong for helpful conversations. We would also like to thank the referee for carefully reading the paper.

\section{Index of Notation}

$\begin{array}{lrlr}\text { bimodule algebra } \mathcal{R}\left(X, Z_{\mathcal{I}}, \mathcal{L}, \tau\right), & 31 & \Phi_{n}, \Psi_{n}: W_{n+1} \rightarrow W_{n}, & 7 \\ \text { birationally 2-geometric, } & 22 & \Phi_{m, n}, \Psi_{m, n}: W_{m} \rightarrow W_{n}, & 9 \\ \text { birationally commutative, } & 10 & \phi_{m, n}, \psi_{m, n}: Y_{m} \rightarrow Y_{n}, & 13 \\ \chi_{n}=\phi_{n} \text { or } \psi_{n}, & 9 & \mathbb{P}=\mathbb{P}\left(A_{1}^{*}\right), & 7 \\ \text { contracted points } S_{n}^{-} \text {and } S_{n}^{+} & 16 & \mathcal{P}(R), \mathcal{P}^{\ell}(R) \text {, set of point modules, } & 6 \\ \text { critically dense set, } & 32 & \mathcal{P}_{r}(R), \mathcal{P}_{r}^{\ell}(R), \text { truncated point modules, } & 6 \\ \text { extremal elements } S_{\infty}^{+}, S_{\infty}^{-}, & 21,22 & Q(A)=D\left[z, z^{-1} ; \tau\right] \text {, graded quotient ring } & 10 \\ \text { function division ring } Q(A)_{0}, & 10 & \text { qgr, quotient category, } \\ \mathcal{K}, \text { the sheaf of rational functions on } Y, & 31 & \text { relevant point scheme data }\left(Y_{n}, \phi_{n}, \psi_{n}\right), & 13 \\ \mathcal{L}_{n}, & 31 & S^{+}=S_{n}^{+}, S^{-}=S_{n}^{-}, Y=Y_{n} \text { for fixed } n, & 19 \\ \text { naïve blowup algebra } R\left(X, Z_{\mathcal{I}}, \mathcal{L}, \tau\right), & 31 & \sigma=\sigma_{n}=\psi_{n} \phi_{n}^{-1}: Y \rightarrow Y, \\ \text { naïve blowup, qgr- } \mathcal{R}\left(X, Z_{\mathcal{I}}, \mathcal{L}, \tau\right), & 32 & \sigma \text {-ample sheaf, } & 32\end{array}$


saturating set, shift of grading $M \rightarrow M[1]$, stable value of $n$, $\alpha^{\star}: \operatorname{Spec} S \rightarrow \operatorname{Spec} R$, map of schemes, truncations $\Phi_{n}^{\prime}(M), \Psi_{n}^{\prime}(M)$ of $M$, twisted coordinate $\operatorname{ring} B(X, \mathcal{L}, \tau)$,

\section{References}

1. M. ARtin, 'Some problems on three-dimensional graded domains', Representation theory and algebraic geometry Waltham, MA, 1995, London Mathematical Society Lecture Note Series 238, (Cambridge University Press, Cambridge, 1997) 1-9 MR 1477464.

2. M. Artin, L. W. Small and J. J. Zhang, 'Generic flatness for strongly Noetherian algebras', J. Algebra 221 (1999) 579-610. MR 1728399.

3. M. Artin and J. T. Stafford, 'Noncommutative graded domains with quadratic growth', Invent. Math. 122 (1995) 231-276. MR 1358976.

4. M. Artin, J. TAte and M. VAn Den Bergh, 'Some algebras associated to automorphisms of elliptic curves', The Grothendieck Festschrift, vol. I, Progress in Mathematics 86 (Birkhäuser Boston, MA, 1990) 33-85. MR 1086882.

5. M. Artin, J. Tate and M. Van den Bergh, 'Modules over regular algebras of dimension 3', Invent. Math. 106 (1991) 335-388. MR 1128218.

6. M. Artin and M. VAn den Bergh, 'Twisted homogeneous coordinate rings', J. Algebra 133 (1990) 249-271. MR 1067406.

7. M. Artin and J. J. Zhang, 'Noncommutative projective schemes', Adv. Math. 109 (1994) 228-287. MR 1304753.

8. M. Artin and J. J. Zhang, 'Abstract Hilbert schemes', Algebr. Represent. Theory 4 (2001) 305-394. MR 1892642.

9. A. I. Bondal and A. E. Polishchuk, 'Homological properties of associative algebras: the method of helices', Izv. Math. 42 (1994) 219-260. MR 1230966.

10. J. Diller and C. FAvre, 'Dynamics of bimeromorphic maps of surfaces', Amer. J. Math. 123 (2001) 1135-1169. MR 1867314.

11. D. Eisenbud, Commutative algebra, with a view toward algebraic geometry, Graduate Texts in Mathematics, 150 (Springer, New York, 1995). MR 1322960.

12. D. Eisenbud and J. Harris, The Geometry of schemes, Graduate Texts in Mathematics 197 (Springer, New York, 2000). MR 1730819.

13. T. Fujita, 'Semipositive line bundles', J. Fac. Sci. Univ. Tokyo Sect. IA Math. 30 (1983) 353-378. MR 0722501.

14. R. Hartshorne, Algebraic geometry, Graduate Texts in Mathematics, 52 (Springer, New York, 1977). MR 0463157.

15. D. S. Keeler, 'Criteria for $\sigma$-ampleness', J. Amer. Math. Soc. 13 (2000) 517-532. MR 1758752.

16. D. S. Keeler, D. Rogalski and J. T. Stafford, 'Naïve noncommutative blowing up', Duke Math. J. 126 (2005) 491-546. MR 2120116.

17. J. Lipman, 'Desingularization of two-dimensional schemes', Ann. Math. (2) 107 (1978) 151-207. MR 0491722.

18. J. C. McConnell and J. C. Robson, Noncommutative Noetherian rings (John Wiley \& Sons, New York, 1987). MR 0934572.

19. C. NĂStăSEscu and F. Van Oystaeyen, Graded ring theory (North-Holland, Amsterdam, 1982). MR 0676974.

20. D. Rogalski, 'Generic noncommutative surfaces', Adv. Math. 184 (2004) 289-341. MR 2054018.

21. D. RogalSkI, 'Idealizer rings and noncommutative projective geometry', J. Algebra 279 (2004) 791-809. MR 2078942.

22. D. Rogalski, 'Gelfand-Kirillov dimension of birationally commutative surfaces', Preprint, 2007, arXiv:0707.3643, Trans. Amer. Math. Soc. to appear.

23. D. Rogalski and J. T. Stafford, 'Naïve noncommutative blowups at zero dimensional schemes', J. Algebra 318 (2007) 794-833. MR 2371973.

24. D. Rogalski and J. J. Zhang, 'Canonical maps to twisted rings', Math. Z. 259 (2008) 433-455. MR 2390090.

25. J. T. StAFFord and M. VAn Den Bergh, 'Noncommutative curves and noncommutative surfaces', Bull. Amer. Math. Soc. 38 (2001) 171-216. MR 1816070.

26. J. T. Stafford and J. J. Zhang, 'Examples in non-commutative projective geometry', Math. Proc. Cambridge Philos. Soc. 116 (1994) 415-433. MR 1291750.

27. D. R. Stephenson and J. J. Zhang, 'Growth of graded Noetherian rings', Proc. Amer. Math. Soc. 125 (1997) 1593-1605. MR 1371143.

28. M. VAN DEN BERGH, 'A translation principle for the four-dimensional Sklyanin algebras', J. Algebra 184 (1996) 435-490. MR 1409223. 
29. A. Yekutieli, 'Dualizing complexes over noncommutative graded algebras', J. Algebra 153 (1992) 41-84. MR 1195406.

D. Rogalski

Department of Mathematics UCSD

La Jolla, CA 92093-0112

USA

drogalsk@math.ucsd.edu
J. T. Stafford

Department of Mathematics

University of Michigan

Ann Arbor, MI 48109-1043

USA

jts@umich.edu

Current address:

Department of Mathematics

Alan Turing Building

University of Manchester

Manchester M13 9PL,

United Kingdom

Toby.Stafford@manchester.ac.uk 